\title{
GEOCHEMICAL CONTROLS ON NUCLEAR MAGNETIC RESONANCE MEASUREMENTS
}

\author{
Rosemary Knight (PI), Kristina Keating (Ph.D. student), Elliot Grunewald (Ph.D. student) \\ Department of Geophysics, Stanford University
}

August 2008

\begin{abstract}
Proton nuclear magnetic resonance (NMR) is used in the Earth Sciences as a means of obtaining information about the molecular-scale environment of fluids in porous geological materials. Laboratory experiments were conducted to advance our fundamental understanding of the link between the NMR response and the geochemical properties of geological materials. In the first part of this research project, we studied the impact of both the surface-area-to-volume ratio $(S / V)$ of the pore space and the surface relaxivity on the NMR response of fluids in sandclay mixtures. This study highlighted the way in which these two parameters control our ability to use NMR measurements to detect and quantify fluid saturation in multiphase saturated systems. The second part of the project was designed to explore the way in which the mineralogic form of iron, as opposed to simply the concentration of iron, affects the surface relaxation rate and, more generally, the NMR response of porous materials. We found that the magnitude of the surface relaxation rate was different for the various iron-oxide minerals because of changes in both the surface-area-to-volume ratio of the pore space, and the surface relaxivity. Of particular significance from this study was the finding of an anomalously large surface relaxivity of magnetite compared to that of the other iron minerals. Differences in the NMR response of iron minerals were seen in column experiments during the reaction of ferrihydrite-coated quartz sand with aqueous Fe(II) solutions to form goethite, lepidocrocite and magnetite; indicating the potential use of NMR as a means of monitoring geochemical reactions. The final part of the research project investigated the impact of heterogeneity, at the pore-scale, on the NMR response. This work highlighted the way in which the geochemistry, by controlling the surface relaxivity, has a significant impact on the link between NMR data and the microgeometry of the pore space.
\end{abstract}

\section{Introduction and Research Objectives}

Nuclear magnetic resonance (NMR) is a measurement technique, developed in the 1940's, that can provide an incredible amount of information about molecular-scale processes and properties in a wide range of materials. Of specific interest in our research is proton NMR, which investigates the molecular-scale environment of hydrogen nuclei. In the Earth sciences proton NMR is most commonly used to measure the response of water in porous geological materials, as a way of obtaining information about the water content and microgeometry of the water-filled regions of the pore space. Despite many years of the use and study of NMR for borehole logging in the petroleum industry there still remain key questions about the basic mechanisms governing NMR relaxation measurements. As a result, the potential of NMR as a means of determining and studying the physical, chemical and biological properties of Earth materials is far from being fully realized. 
The goal of our research was to advance the fundamental understanding of the link between the NMR response and the geochemical properties of geological materials. While it is well established that the presence of iron is a dominant factor in determining the NMR response of rocks and soils, we identified a need to understand the effects of the specific mineralogic form of the iron and the distribution of the iron on NMR relaxation mechanisms. In addition the current models of NMR relaxation times in porous materials greatly oversimplify both the geometry of the pore system and the related interactions between the protons in the pore fluid and the paramagnetic iron. We proposed a three-year laboratory-based study of NMR relaxation time measurements where sample composition, pore structure and geochemical conditions were carefully controlled or determined. Some of our previous work had raised the intriguing possibility that NMR measurements could be used to detect or monitor geochemical processes. Therefore we proposed to measure the NMR response of geologic systems both at chemical equilibrium and as the chemistry of the system changed due to changing redox conditions and ongoing biological activity. We proposed to work initially with systems that could be described as spatially homogeneous, given the time scale of the NMR measurement, and then investigate the role of pore-scale variability in geochemical properties in determining NMR relaxation times.

Central to the proposed research was the completion in 2002 of a new Environmental Geophysics Laboratory in Earth Sciences at Stanford University and the acquisition of a Maran Ultra NMR core analyzer (purchased with funds from the National Science Foundation, with matching funds from Stanford). With these new laboratory facilities, we were able to undertake an extensive experimental and theoretical study of the fundamental geochemical controls on the NMR response of geological materials.

We encountered one significant obstacle in completing our research and that was the resignation in Year 1 of the Research Associate on the project. Rather than supporting a senior scientist and completing the work in the proposed time frame of three years, the funding was used to support a graduate student and the project extended (through no-cost extensions) over five years.

The motivation for our proposed research is a recognized need to conduct the basic research required to advance the use of NMR in a wide range of applications in the earth sciences. Such applications could involve NMR measurements made in the laboratory using commercially-available spectrometers, in boreholes using NMR logging devices, or from the surface of Earth using the recently developed surface-loop system. Regardless of the mode of data collection, NMR can potentially provide a wealth of information about pore-scale physical, chemical and biological properties and processes in Earth materials.

\section{The NMR Measurement}

The proton NMR experiment begins with the exposure of a sample to a constant external magnetic field; this causes the alignment of the magnetic moments of the protons in the pore water. A radio-frequency pulse, at the Larmor frequency of the protons, is then used to excite some of the protons to a higher energy state, and the magnetization $M$ is monitored over time $t$ as the system returns or "relaxes" to equilibrium.

Let us first consider the response of water in a single pore. There are three different relaxation mechanisms, each with its own characteristic relaxation time, that contribute to the observed relaxation time - or relaxation rate - of the water in the pore. There is the relaxation of the bulk fluid, with a corresponding relaxation time denoted as $T_{b}$. This is the relaxation time 
that would be observed for a sample of the pore water contained in a beaker or test tube, unaffected by an enclosing pore space. The presence of the pore surface results in an additional relaxation mechanism due to the proximity of the liquid molecules to the solid surface. The relaxation time associated with this mechanism is referred to as the surface relaxation time $T_{\mathrm{s}}$. In the case referred to as "fast diffusion" (treated by Brownstein and Tarr (1979)), which assumes that all the protons travel to and relax at the solid surface in the time interval of the NMR experiment, the surface relaxation rate is given by the following expression:

$$
\frac{1}{T_{s}}=\rho\left(\frac{S}{V}\right)
$$

where $S / V$ is the surface-area-to-volume ratio of the pore space and $\rho$, the surface relaxivity, is a measure of the ability of the solid surface to enhance the relaxation rate of the bulk pore fluid. The third mechanism is the relaxation due to diffusion in a magnetic field gradient $G$. An internal magnetic field gradient can arise due to differences in the magnetic susceptibility across a sample (e.g., the contrast between the solid and the pore fluid); an external field gradient can also be experimentally imposed. This diffusion-related relaxation mechanism is represented by the relaxation time $T_{d}$ and is described by the following expression:

$$
\frac{1}{T_{d}}=\frac{D}{12}\left(\gamma G t_{E}\right)^{2}
$$

where $D$ is the diffusion coefficient, $\gamma$ is the gyromagnetic ratio of the proton and $t_{E}$ is the echo spacing of the radio-frequency pulses used for data collection. In some measurements this diffusion term is close to 0 ; in others it becomes the dominant relaxation mechanism.

Much of the interest in the use of NMR to investigate the pore-scale properties of geologic materials has focused on the case where the diffusion term is negligible. Then the following expression describes the relaxation time, $T_{2}$, for the transverse component of $M$, for a single pore:

$$
\frac{1}{T_{2}}=\frac{1}{T_{b}}+\rho\left(\frac{S}{V}\right)
$$

In the case where the magnetic susceptibility is non-zero, the diffusion term cannot be neglected and $T_{2}$ is then given by:

$$
\frac{1}{T_{2}}=\frac{1}{T_{b}}+\rho\left(\frac{S}{V}\right)+\frac{D}{12}\left(G \gamma t_{E}\right)^{2}
$$

If we now consider water contained in a porous material with a distribution of pore sizes, it is generally assumed that the above expression applies to each pore size and that each pore size acts in isolation in contributing to the NMR measurement. The magnetic relaxation curve is represented by a sum of exponentials, one term for each pore size with a unique relaxation time here denoted by $T_{i}$ : 


$$
M(t)=\sum_{i} A_{i} \exp \left(-t / T_{i}\right),
$$

where the amplitude $A_{i}$ is proportional to the number of water molecules (protons) in the sample with the relaxation time $T_{i}$. $A_{i}$ is often plotted versus $T_{i}$ yielding a distribution of relaxation times. If the diffusion term can be neglected, the distribution of relaxation times can then be equated to the pore size distribution of the sample. This interpretation assumes 1) that the pore space is composed of spherical and isolated pores and 2) that $\rho$ is constant and known throughout the sample. The arithmetic mean of the log relaxation times from equation (5), $T_{2 M L}$, is used to represent the average relaxation times for the entire sample. If the relaxation time of the bulk fluid is known, the average surface relaxation time and average diffusion relaxation time can then calculated from $T_{2 M L}$; these values are assumed to be averages taken over the entire sample.

The link between the NMR response and the properties of geologic materials is primarily through the surface relaxation time, which is determined by both $\rho$ and $S / V$. The first key question that we addressed: How does variation in $\rho$ and $S / V$ impact the NMR response of different fluids in the same porous material?

The next stage of our research focused on the control on $\rho$. It is generally accepted that the dominant mechanism for surface relaxation in geological materials is the dipolar coupling between the protons in the diffusing water molecules and paramagnetic surface sites. In geologic materials the paramagnetic ions that are most abundant are $\mathrm{Fe}(\mathrm{III})$, but also include $\mathrm{Mn}(\mathrm{II})$, $\mathrm{Fe}(\mathrm{II})$ and $\mathrm{Cu}(\mathrm{II})$. While previous studies had demonstrated a strong dependence of $\rho$ on the presence of paramagnetic sites, they all worked with very simple systems, and left many issues needing to be addressed. The second question we addressed: how does the mineralogic form of the iron affect $\rho$ and, more generally, NMR relaxation?

The other parameter linking NMR relaxation measurements to the properties of geologic materials is $S / V$. Most current models assume that relaxation occurs in isolated pores. Based on this starting assumption, interpretation of NMR data commonly involves using the distribution in relaxation times to obtain a distribution in $S / V$ values, which is used to obtain a distribution in pore sizes. However, given the fact that a water molecule can travel as far as $100 \mu \mathrm{m}$ within the time scale of the NMR measurement at room temperature, the parameter $S / V$ is most likely an effective or averaged value determined by the geometry of the pores visited by the diffusing molecules. The third question that we addressed: How does diffusion of protons between heterogeneous pores affect the NMR relaxation measurement?

\section{Completed Research}

All NMR measurements were made at $2.2 \mathrm{MHz}$ with the Maran Ultra NMR analyzer. $T_{2}$ was measured using the Carr-Purcell-Meiboom-Gill (CPMG) pulse sequence; 32000 linearlyspaced data points were collected for each measurement with spacing set by the echo time $\left(t_{E}\right)$. The data were stacked 100 times to improve the signal to noise ratio and the measurements were repeated for multiple $t_{E}$. To invert the data, we used Tikhonov regularization and linear programming algorithms as implemented by Whittall and MacKay (1989) and Whittall et al. (1991). This method fits 200 exponentially spaced $T_{2}$ values ranging from $1 \mathrm{~ms}$ to $10 \mathrm{~s}$ to the dataset yielding the relaxation time distribution. 


\section{The NMR Response of Multiphase Saturated Systems}

The first set of experiments involved a comparison of the NMR response of three different pore fluids - toluene, bis(2-ethylhexyl) phthalate (BEHP), and water - contained in the same porous material; where the material was saturated with a single pore fluid and with mixtures of water and a second fluid. NMR measurements were made on water, toluene or BEHP in a number of different sand-clay mixtures to understand the role of clay (i.e. $S / V$ ) and iron content (i.e. ) on the $\mathrm{T}_{2}$ of each fluid; we then made NMR measurements on mixtures of water and the contaminants in each sand-clay mixture. Our ability to resolve the NMR response of the contaminant from that of water, and to quantify the amount of the contaminant varied significantly with the clay and iron content of the sand-clay mixtures. Detection limits as low as $3 \%$ contaminant (i.e., percent of the pore fluid, by weight) were observed in some sand-clay mixtures. Our results suggest that NMR measurements could quantify toluene if some clay is present, but are unlikely to be useful for quantifying BEHP. These results show clearly the dominant role of $S / V$ and $\rho$ in determining 1) the response of single fluids and 2) our ability to resolve the presence of individual fluids in multiphase saturated systems.

\section{Peer Reviewed Publications:}

Bryar, T.R. and Knight, R.J., NMR Relaxation Measurements to Quantify Immiscible Organic Contaminants in Sediments, Water Resour. Res., 44, W02401, doi:10.1029/2006WR005635, 2008.

\section{The Impact of Iron Mineralogy on Relaxation Times}

To advance the fundamental understanding of the link between the NMR response and the geochemical properties of geological materials we completed three studies investigating the effect of iron (hydr)oxide minerals on NMR relaxation. We focused on iron (hydr)oxides because they are ubiquitous in the environment and, due to their high reactivity, can strongly influence the mobility and bioavailability of organic and inorganic contaminants. The first two studies measured the response at chemical equilibrium and focused on the common iron-oxide minerals: ferrihydrite, goethite, lepidocrocite, hematite, and magnetite. The third study focused on measuring the NMR response during iron (hydr)oxide mineralization processes. Although we initially proposed to measure systems in which iron minerals transformed due both to changing redox conditions and to biological activity, understanding the iron (hydr)oxide reactions in the absence of biological activity required much more intensive chemical analysis then originally anticipated. We thus limited the scope to focus on systems where iron minerals changed due to changing redox conditions. The results from these three studies were presented at four conferences and are contained in three publications as listed below:

\section{Conference Presentations:}

Keating, K., R. Knight, and T. Borch (2005), Proton Nuclear Magnetic Resonance: A Novel Approach for Monitoring In Situ Iron Mineralization Processes, Eos Trans. AGU, 86(18), Jt. Assem. Suppl., Abstract NS51B-05.

Keating, K., and R. Knight (2006) The Influence of Iron Oxides on Nuclear Magnetic Resonance Measurements, SEG Hydrogeophysics Workshop, Vancouver.

Keating, K., and R. Knight (2006) The Effect of Magnetite on NMR Relaxation Rates: A 
Laboratory Study, 3rd International Magnetic Resonance Sounding Workshop, Madrid.

Keating, K., R. Knight, and K. J. Tufano (2007), Using Nuclear Magnetic Resonance to Monitor Iron Mineralization Processes, Eos Trans. AGU, 88(23) Jt. Assem. Suppl., Abstract NS51A04.

Peer Reviewed Publications:

Keating, K., and R. Knight, (2007) A Laboratory Study to Determine the Effects of Iron-Oxides on Proton NMR Measurements. Geophysics, 72(1) E27-32.

Keating, K., and R. Knight, (2008) A laboratory study of the effects of Magnetite on NMR Relaxation Rates. Journal of Applied Geophysics, in press.

Keating, K, R. Knight, and K. J. Tufano, (2008) Nuclear magnetic relaxation measurements as a means of monitoring iron mineralization processes, Geophysical Research Letters, submitted.

In the study reported in Keating and Knight (2007), we investigated the effect of the presence, and mineralogic form of, iron on measured NMR relaxation rates. Five samples of quartz sand were coated with ferrihydrite, goethite, hematite, lepidocrocite, and magnetite. The relaxation rates for these iron-oxide-coated sands saturated with water were measured and compared to the relaxation rate of quartz sand saturated with water. We found that the presence of the iron oxides led to increases in the relaxation rates by increasing the surface relaxation rate. The magnitude of the surface relaxation rate was different for the various iron-oxide minerals because of changes in both the surface-area-to-volume ratio of the pore space, and the surface relaxivity. Of particular significance from this study was the finding of the anomalously large surface relaxivity of magnetite compared to that of the other iron minerals. The relaxation rate of the magnetite-coated sand was further increased because of internal magnetic field gradients caused by the presence of magnetite. We concluded that both the concentration and mineralogical form of iron can have a significant impact on NMR relaxation behavior.

In Keating and Knight (2008, in press), we conducted a laboratory study to further explore the effect of magnetite on the NMR relaxation behavior. In this study we looked at the effect of magnetite concentration and grain size on NMR relaxation rates of sand mixtures. We measured mixtures of quartz and three different forms of magnetite: a powdered synthetic magnetite; a small-grained, natural magnetite; and a large-grained, natural magnetite. The powdered synthetic magnetite was mixed with quartz in five concentrations ranging from 0.14 to $1.4 \%$ magnetite by weight; both sizes of natural magnetite were mixed with quartz in concentrations of 1 and $2 \%$ magnetite by weight. The NMR responses of the water-saturated samples were measured and used to calculate four averaged relaxation rates for each magnetite concentration: the total mean log, bulk fluid, surface, and diffusion relaxation rates. The results of this study showed that: 1) surface relaxation was the dominant relaxation mechanism for all samples except the powdered synthetic magnetite sample containing $1.4 \%$ magnetite; 2 ) the surface relaxivity is a function of the fraction of the surface area in the sample composed of magnetite; 3 ) there is no clear dependence of the diffusion relaxation rate on the concentration of magnetite.

In the third study, (Keating, Knight, and Tufano, 2008, submitted), we conducted experiments in which columns containing ferrihydrite-coated quartz sand reacted with aqueous $\mathrm{Fe}$ (II) solutions to form goethite, lepidocrocite and magnetite. An observed increase in the volume of water relaxing with long relaxation times in the NMR relaxation time distribution corresponds to the formation of goethite and lepidocrocite; a decrease in the average (mean log) relaxation time, and a broadening of the relaxation time distribution, corresponds to the 
formation of magnetite. These results indicated that NMR relaxation times are sensitive to changes in iron mineralogy and illustrate the potential use of NMR for monitoring iron mineralization processes.

\section{The Impact of Interpore Diffusion}

The conventional interpretation of NMR relaxation data assumes a model of isolated pores in which each proton samples only one pore type and the relaxation-time distribution can be directly scaled to represent a pore-size distribution. In realistic geologic materials, however, heterogeneous pores are often very well connected and protons may readily diffuse between dissimilar pores during the NMR measurement. In this case, interpore diffusion or "pore coupling" complicates estimates of pore geometry, and the meaning of the relaxation time distribution in such systems is not well understood. In order to improve our understanding of the link between the NMR relaxation response and pore geometry, we completed a laboratory study focusing on NMR relaxation in materials with a bimodal pore-size distribution. By altering the surface geochemistry, we synthesized a range of samples in which the degree of pore coupling varies. Laboratory NMR measurements on this collection of samples thus allowed us to directly investigate the effects of pore coupling on the NMR relaxation response.

For this study, we used grain packs of silica gel which have a bimodal pore size distribution comprised of intragranular microporosity (within the grains) and larger intergranular macropores (between the grains). In order to alter the degree of pore coupling between samples, we sorbed varied amounts of paramagnetic iron $\left(\mathrm{Fe}^{3+}\right)$, to the surfaces of the gels, thus changing the surface relaxivity of the pores. NMR measurements were first collected on fully saturated samples and then again after the samples had been centrifuged to remove water from the large intergranular pores; thus, we were able to obtain independent measurements of the intragranular porosity.

We hypothesized that for samples with low iron concentrations and a low surface relaxivity, relaxation would occur slowly and pore coupling would be strong. Upon sorbing increasing concentrations of $\mathrm{Fe}^{3+}$ to the gel surfaces, we expected relaxation to occur more quickly, reducing the ability of protons to diffuse between pore types during the measurement, and so reducing the degree of pore coupling. NMR measurements on this range of samples thus provided an opportunity to directly explore the effect of interpore diffusion over a range of coupling strengths.

Relaxation time distributions for each of the four samples are shown in Figure 1 with iron concentrations increasing from left to right; the top panels show results for measurements at full saturation, while the lower panels show results after centrifuging. 

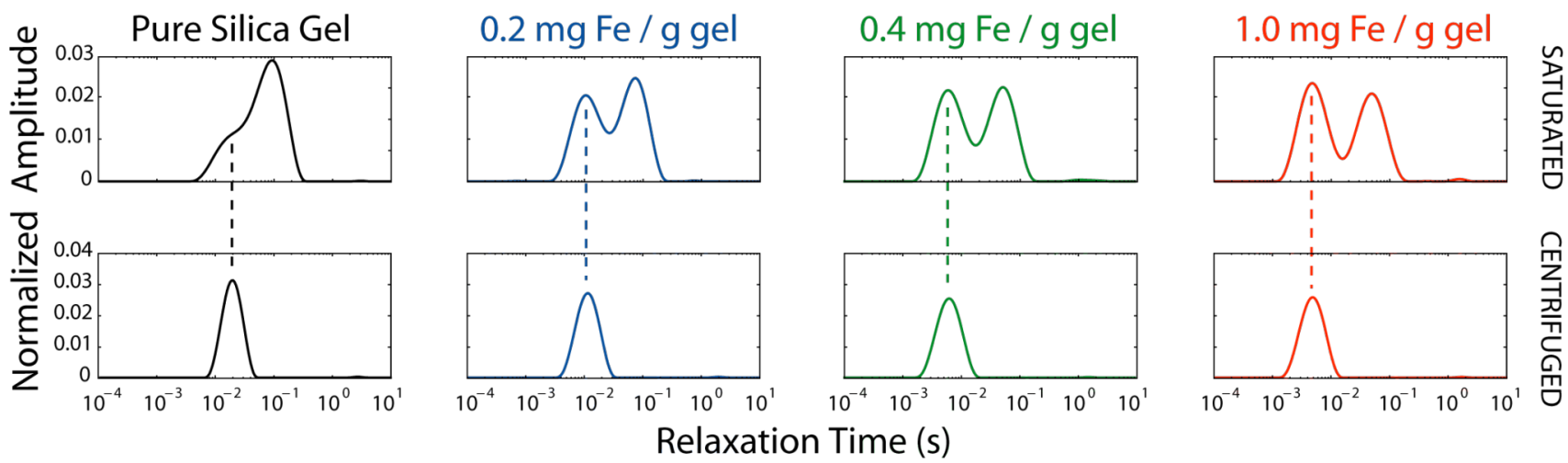

Figure 1. NMR relaxation time distributions for the four silica gel samples. Iron concentrations increase from left to right with warmer colors. Top panels are for saturated samples and bottom panels are for centrifuged samples. Dashed lines illustrate that short relaxation times correspond exactly to the intragranular microporosity.

As expected, we observe reduced pore coupling and increased separation of the two relaxation time peaks for samples with higher iron concentrations. We note that the short-time relaxation peaks correspond exactly to the relaxation time associated with the intragranular microporosity as evidenced by independent measurements on the centrifuged samples.

Therefore, the location of the shortest relaxation time peak is independent of the degree of pore coupling. The long-time relaxation time peaks, however, become more closely merged with the short-time peaks for samples with stronger pore coupling. Additionally, we observe that the ratio of total microporosity to macroporosity volume $(\sim 1.2)$ represented by the relative size of the two peaks is underestimated in samples with stronger pore coupling.

These observations are supported by a conceptual model in which relaxing protons move freely between the two pore types before relaxing. The leftward merging of the long relaxation time peak for samples with low iron concentrations occurs because protons which originate in the macropores have time to diffuse into the intragranular micropores before relaxing. Therefore, these protons exhibit an averaged relaxation time which is shorter than if the pores were isolated. Likewise, when relaxation occurs slowly, some protons are able to leak out of the smaller intragranular pores before relaxing and so also relax at an averaged rate. For the most heavily iron-treated samples, relaxation occurs rapidly enough that the majority of the protons originating in the intragranular pore relax before they can leak outward. Therefore, the relaxation-time distribution more closely represents the true pore-size distribution for the samples with a higher surface relaxivity value. These results demonstrate that pore coupling can dramatically skew the interpretation of NMR measurements and that the degree of pore coupling may be influenced by the pore surface geochemistry. Our findings from this study have been presented at the following geophysics conferences and are currently being prepared for submission to Geophysical Research Letters:

\section{Conference Presentations}

Grunewald, E. and R. Knight, (2007) A laboratory study of NMR relaxation times and pore coupling in heterogeneous porous media, Eos Trans. AGU, 88(52), Fall Meet. Suppl., Abstract NS31B-0378.

Grunewald, E. and R. Knight, (2008) The link between NMR relaxation times and pore size in heterogeneous porous media: the effect of interpore diffusion, Schlumberger Water Services Meeting, Stanford University. 


\begin{abstract}
NMR Relaxation Measurements
to Quantify Immiscible Organic Contaminants in Sediments
\end{abstract}

Traci R. Bryar and Rosemary J. Knight

Department of Geophysics, Stanford University, Stanford CA

\begin{abstract}
An edited version of this paper was published by AGU. Copyright 2008 American Geophysical Union.
\end{abstract}

The full citation is given below:

Bryar, T.R. and Knight, R.J., NMR Relaxation Measurements to Quantify Immiscible Organic Contaminants in Sediments, Water Resour. Res., 44, W02401, doi:10.1029/2006WR005635, 2008.

To view the published open abstract, go to http://dx.doi.org and enter the DOI. 


\begin{abstract}
Laboratory $\mathrm{NMR}_{2}$ relaxation measurements at $2 \mathrm{MHz}$ were used to quantify the amount of toluene and bis(2-ethylhexyl) phthalate (BEHP) in the presence of water in sand-clay mixtures. NMR measurements were made on water, toluene or BEHP in each sand-clay mixture to understand the role of clay and iron content on the $\mathrm{T}_{2}$ of each fluid; we then made NMR measurements on mixtures of water and the contaminants in each sand-clay mixture. Our ability to resolve the NMR response of the contaminant from that of water, and to quantify the amount of the contaminant varied significantly with the clay and iron content of the sand-clay mixtures. Detection limits as low as $3 \%$ contaminant (i.e., percent of the pore fluid, by weight) were observed in some sand-clay mixtures. Our results suggest that NMR measurements could quantify toluene if some clay is present, but are unlikely to be useful for quantifying BEHP.
\end{abstract}

Index terms: 1831, 1895, 3929

Keywords: NMR, BTEX, LNAPL, DNAPL, contaminant 


\section{INTRODUCTION}

There is growing interest in the use of non-invasive geophysical methods as a means of detecting subsurface organic contaminants. Proton nuclear magnetic resonance (NMR) is one method that has potential to detect non-aqueous phase, liquid organic contaminants due to its sensitivity to the presence of any hydrogen-bearing fluid.

Borehole NMR relaxation measurements have been made in the petroleum industry since the early 60's to detect hydrocarbons and estimate reservoir porosity [Brown and Gamson, 1960]. Field measurements obtained with a surface loop NMR instrument have been used to estimate water content and the average grain size distribution with depth in groundwater aquifers [Schirov et al., 1991; Legchenko et al., 1997].

Previous studies have shown that laboratory NMR measurements can identify the presence of hydrocarbons when both water and a hydrocarbon are present; e.g., gasoline and water in sandstones [Hedberg et al., 1993], and kerosene and water in sandstones [Straley et al., 1995]. In earlier work we established that toluene could be quantified in some sediments by NMR relaxation measurements; however, sediment lithology and iron content affected the ability to separate the toluene signal from that of water [Bryar et al., 2001]. More recently, NMR diffusion measurements using field gradients have reliably discriminated oil from water [Flaum et al., 2005].

Our interest in this study is to assess the use of NMR relaxation measurements to quantify immiscible, organic liquids in sand-clay mixtures in the presence of water. Although this is a laboratory study we collect data of the same signal-to-noise ratio attainable with borehole and surface NMR instruments. We use a model system to assess the potential of these field instruments to quantify immiscible organic fluids at 
contaminated sites. We selected toluene and bis(2-ethylhexyl) phthalate (BEHP) as the contaminants. Toluene represents the BETX family of ground water contaminants (benzene, ethylbenzene, toluene, and xylenes) found in association with leaking underground gasoline and diesel fuel storage tanks [Swobada-Colberg, 1995]. BEHP, one of the 10 most commonly occurring ground water contaminants, is listed as a priority one hazardous substance [Lucius et al., 1992], and has been deemed undetectable by noninvasive geophysical methods [Olhoeft, 1992]. We focus on the effects of clay content and concentration of iron on mineral surfaces on our ability to accurately quantify these two contaminants.

\section{NMR RELAXATION}

Proton NMR detects the $\mathrm{H}$ atoms of liquids [e.g., Abragam, 1961]. When the liquid experiences a static magnetic field $\left(\mathbf{B}_{\mathbf{0}}\right)$, the magnetic moment of each ${ }^{1} \mathrm{H}$ nucleus orients with respect to the field, producing a net nuclear magnetization (M). In the NMR experiment a short burst, or pulse, of radio-frequency (rf) power is applied at a discrete frequency (Larmor frequency, $\omega_{0}$ ) to excite the magnetic moments away from their equilibrium orientations. Once the rf power is turned off the magnetization, $\mathbf{M}$, returns (or relaxes) back to its equilibrium position. The signal measured during the relaxation is $\mathbf{M}_{\perp}$, the component of $\mathbf{M}$ perpendicular to $\mathbf{B}_{\mathbf{0}}$. The change in net magnetization is detected by induction in the $\mathrm{rf}$ coil; the measured signal intensity $(I)$ is proportional to the magnitude of $\mathbf{M}_{\perp}$.

For bulk liquids the relaxation follows exponential decay: 


$$
I(t)=I(0) e^{-t / T_{2 b}},
$$

where the inverse of the rate constant is referred to as the bulk fluid relaxation time $\left(T_{2 \mathrm{~b}}\right)$. The initial signal intensity, $I(0)$, is proportional to the amount of liquid; more specifically, $I(0)$ is proportional to the total moles of $\mathrm{H}$ atoms in the liquid sample. The magnitude of $T_{2 \mathrm{~b}}$ for a liquid, determined by dipole-dipole molecular interactions, decreases with increasing fluid viscosity or increasing concentration of paramagnetic species such as dissolved oxygen or $\mathrm{Mn}^{2+}$ ions [Bloembergen et al., 1948].

When a liquid is confined in a pore, the relaxation time is usually less than $T_{2 \mathrm{~b}}$. This reduction in relaxation time has been attributed to the effect of the surface of the solid on $\mathbf{M}$ of the liquid's $\mathrm{H}$ nuclei, due either to a change in the properties of the liquid adjacent to the solid surface [Torrey, 1956; Korringa, 1956], or to the presence of relaxation sites on the surface [Senturia and Robinson, 1970; Brownstein and Tarr, 1979]. The relaxation time associated with this mechanism is referred to as the surface relaxation time $T_{2 S}$. In the case referred to as fast diffusion [Brownstein and Tarr, 1979], the surface relaxation rate constant $1 / T_{2 \mathrm{~S}}$ is given by:

$$
\frac{1}{T_{2 S}}=\rho\left(\frac{S}{V_{p}}\right),
$$

where $S / V_{p}$ is the surface-area-to-volume ratio of the pore space and $\rho(\mu \mathrm{m} / \mathrm{s})$, the surface relaxivity, can be interpreted as the ability of the pore surface to enhance 
relaxation. Fast diffusion implies that the rate of surface relaxation is less than the rate of diffusive mixing within the pore [Banavar and Schwartz, 1987]. The primary control on $\rho$ in geological materials is known to be the concentration of paramagnetic species such as $\mathrm{Fe}(\mathrm{III})$ and $\mathrm{Mn}(\mathrm{II})$, with $\mathrm{Fe}(\mathrm{III})$ being the most common paramagnetic species contained in minerals in the near-surface region of the earth. The magnitude of $\rho$ has been found to increase with iron and manganese content [Foley at al., 1996; Bryar et al., 2000] and is also expected to be higher in systems where the liquid wets the solid surface [e.g., Brown and Fatt, 1956].

For a liquid in a porous geologic material, with a range of pore sizes, multiexponential relaxation is observed:

$$
I(t)=\sum_{i} A_{i} \exp \left(-t / T_{i}\right)
$$

where $\sum A_{i}$ is proportional to the total moles of $\mathrm{H}$ atoms in all pores in the sample. This results in a distribution of relaxation times, assumed to represent the distribution of pore sizes in the material [Kenyon, 1992]. The parameter most commonly reported, and used in this study, to compare the NMR response of different materials is the logarithmic mean relaxation time $T_{2}$ ML. $T_{2}$ ML is the weighted mean of the log of the $T_{i}$ values (weighted by $\left.A_{i}\right)$. For symmetric peaks, $T_{2}$ ML will indicate the center of the peak.

The following expression describes $T_{2 \mathrm{ML}}$ of a liquid in a porous material in terms of the bulk fluid and surface relaxation times constants, 


$$
\frac{1}{T_{2 M L}}=\frac{1}{T_{2 b}}+\frac{1}{T_{2 S}}
$$

where $T_{2 \mathrm{~S}}$ is an averaged value for the porous medium equated to $S / V_{p} / \rho$ with $S / V_{p}$ and $\rho$ also now taken to be averaged values. This expression can include a third term, to account for the effect of internal field gradients $(G)$ on the diffusing hydrogen nuclei [Brown and Fantazinni, 1993]. However, in this study we found experimentally that $G$ was negligible, so (4) describes the NMR response of our samples.

We are interested in the case where both water and a liquid contaminant co-exist in the pore space, so both H-containing liquids contribute to the recorded signal. The signal intensity as a function of time becomes:

$$
I(t)=\sum\left(A_{i} e^{-t / T_{2_{i}}}\right)_{\text {organic conta min ant } H}+\sum\left(A_{i} e^{-t / T_{2_{i}}}\right)_{\text {water } H}
$$

where $\sum A_{i}$ for the contaminant is proportional to the moles of contaminant $\mathrm{H}$, and $\sum A_{i}$ for water is proportional to the moles of water H. Each fluid will have a distribution of relaxation times, characterized by a mean relaxation time, $T_{2} \mathrm{ML}$. The $T_{2 \mathrm{ML}}$ value for each fluid is described by (4), so depends on the properties of the pore fluid and the region of the porous medium occupied by the fluid.

The challenge we face, in using NMR for contaminant detection, is being able to distinguish between contaminant $\mathrm{H}$ and water $\mathrm{H}$ in a geologic material. In order to do this, the mean relaxation times for the two immiscible liquids, when contained in the 
porous medium, must be sufficiently different. This requires differences in one or more of the parameters in (4) that determine $T_{2 \mathrm{ML}}$. Exploring these differences and, therefore, our ability to use NMR as a means of detecting and quantifying organic contaminants is the focus of this laboratory study. We limit our study to toluene and BEHP in sand and clay samples with varying clay and iron content.

\section{DESCRIPTION OF SAMPLES}

The baseline sand used in this study was a fine-grained foundry-grade sand (obtained from Wedron Silica Co., IL) with $0.1 \%$ clay content. The median grain size is $0.13 \mathrm{~mm}$ [Chan and Knight, 2001]. The grain density of the sand is $2.67 \pm 0.02 \mathrm{~g} / \mathrm{cm}^{3}$, as determined by helium pycnometer. The baseline clay used was a low defect kaolinite, (referred to as KGa-1b) acquired from the Source Clays Repository (Department of Geological Sciences, University of Missouri, Columbia, MO). The grain density is 2.62

$\mathrm{g} / \mathrm{cm}^{3}$ [Li et al., 2001]. We refer to these materials as the "untreated" sand and clay. Additional sands and clays with iron oxyhydroxide $(\mathrm{FeOOH})$ coatings were prepared from the untreated sand and clay using the method of Grantham et al. [1997]. A mixture of sand or clay and aqueous $\mathrm{FeSO}_{4}$ was oxidized with $30 \% \mathrm{H}_{2} \mathrm{O}_{2}$, left standing overnight, rinsed with de-ionized water $(18 \mathrm{M} \Omega-\mathrm{cm})$, then oven-dried at $100{ }^{\circ} \mathrm{C}$. The dried $\mathrm{FeOOH}$-coated clays were placed in a plastic bag and gently tapped with a rubber mallet to separate the individual particles. The prepared sands and clays were exposed to the ambient lab humidity to return to the same water-wet state as the untreated sand and clay. Although contact angles were not measured, observations of the behavior of a drop 
of each fluid on the solids clearly showed the surfaces were water wet; that is, water spread out on the surfaces whereas toluene and BEHP beaded. These treatments produced three batches of sand (sand A, sand B, sand C), and three of clay (clay A, clay $\mathrm{B}$, clay $\mathrm{C}$ ), coated with varying amounts of iron; the variation in iron was obtained by using different concentrations of aqueous $\mathrm{FeSO}_{4}$. The treatments of the sands and clays produced no measurable difference in grain densities.

Grain densities, iron contents and specific surface areas $\left(S_{s}, \mathrm{~m}^{2} / \mathrm{g}\right)$ of all the sands and clays are listed in Table 1. The iron contents were determined by ICP analysis of aqua regia leachate (ASL Chemex, Reno, NV). Specific surface areas were determined, using the Brunauer-Emmett-Teller (BET) equation [Brunauer et al., 1938], from krypton gas isotherms for the sands and from nitrogen gas isotherms for the clays. (While nitrogen is typically used for surface area measurements, the use of krypton is recommended to improve the measurement accuracy for low surface area materials.)

Using the pure sands and clays, four types of mixed sand-clay samples were made where clay content varied from 0 to $100 \%$ of the solid phase by weight. The four types of samples included mixtures of the untreated sand and clay to form samples referred to as the untreated sand-clay mixtures; mixtures of sand A with clay A, to form the A sandclay mixtures; mixtures of sand B with clay B, to form the B sand-clay mixtures; and mixtures of sand $\mathrm{C}$ with clay $\mathrm{C}$, to form the $\mathrm{C}$ sand-clay mixtures. To ensure that the clay fraction and the porosity were known exactly, each NMR sample was prepared from the individual single-component sands and clays; thus at any one time, only enough sample was mixed to fill one NMR sample cell. 


\section{NMR EXPERIMENTAL DETAILS}

In order to make relaxation measurements in a way that allowed us to accurately determine sample porosity and saturation, we machined thick-walled cylindrical Teflon cells, with a cap capable of compressing the sample to $\sim 18 \mathrm{~cm}^{3}$ (approximately one half of the cell's total volume) as it is screwed shut. The volume of each cell was determined by weighing the cell (with cap fully shut) empty and filled with water. These cells, which held the sample, were placed in the bottom of the standard NMR $40 \mathrm{cc}$ test tube for measurement.

$T_{2}$ relaxation data were collected at $25.0^{\circ} \mathrm{C}$ using a $2 \mathrm{MHz}$ proton Maran Ultra NMR spectrometer (Resonance Instruments). The standard Carr-Purcell-Meiboom-Gill (CPMG) pulse sequence was used with an echo time $\left(t_{E}\right)$ of $200 \mu$ s and a recovery time of $5 \mathrm{~s} ; 32,000$ linearly-spaced data points were collected for each experiment. A short echo time was used to increase data density at the beginning of the relaxation curve and to ensure that $\mathrm{G}$ has no effect on equation 4. For each sample, 100 equivalent scans were stacked and the data were phase corrected. The noise was determined from the channel with zero signal. For these experiments the signal-to-noise ratio was between 100 and 170. Note that $T_{2}$ measurements using $t_{\mathrm{E}}$ of 500 and $800 \mu \mathrm{s}$ were completed for three samples. For untreated sand and clay, G was approximately zero. For samples with iron the relaxation time changed very little (i.e., only slightly more than experimental error), indicating that $\mathrm{G}$ was small.

All NMR measurements of the liquids in the porous samples yielded a multiexponential decay described by (5). To decrease the processing time of the NMR data, 
the measured decay curves were divided into 400 exponentially-spaced time bins and the points within each bin were averaged [Whittall, 1994]; error for each of the 400 averaged data points was calculated from the noise of the raw data and the square root of the number of points averaged. In order to extract a distribution of relaxation times, this averaged magnetization decay curve was fit to a distribution of $160 T_{2}$ values (ranging from $1 \mathrm{~ms}$ to $10 \mathrm{~s}$ ) using Tikhonov inversion as implemented by Whittall and Mackay [1989]. The regularizing parameter was selected such that the data were fit to approximately one standard deviation.

\section{SAMPLES CONTAINING ONE FLUID}

\section{$T_{2}$ Measurements on Bulk Fluids}

We first measured the bulk relaxation times of the water, toluene, and BEHP by filling the Teflon NMR cells with $\sim 18 \mathrm{cc}$ of liquid. Triplicate measurements were made on three samples of each liquid, yielding an estimate of $T_{2 \mathrm{~b}}$ measurement uncertainty of 3$4 \%$. The bulk relaxation time measured for bulk water was $2.40 \pm 0.09 \mathrm{~s}$, for toluene was $1.37 \pm 0.04$ and for bulk BEHP was found to be $0.076 \pm 0.003 \mathrm{~s}$. The fluids are in equilibrium with air and the bulk values reflect the effect of dissolved oxygen. In an anaerobic environment, the $T_{2 \mathrm{~b}}$ values would be slightly higher. The very low value of $T_{2 \mathrm{~b}}$ for BEHP is due to the high viscosity of the liquid (i.e., 80 times that of water). 


\section{$\mathbf{T}_{2}$ Measurements on Individual Fluids in Sand-Clay Mixtures}

This set of experiments was designed to develop an understanding of the differences between the NMR responses of the two contaminants and water, when each was present as a single, saturating phase in sand-clay mixtures. If a contaminant has the same NMR response as water, it would be unlikely that we would be able to use NMR to discriminate between the contaminant and water in a system containing both fluids.

To prepare a sample for NMR measurement, a measured amount of the pore fluid (water, toluene or BEHP) was first added to an NMR cell, followed by the solid phase; the sample was compressed and the lid screwed shut. The porosity of each prepared NMR sample was calculated from the mass of the solid components (measured before addition to the NMR cell), using the volume of the cell and the density of the sand and clay. Working with water and toluene as the pore fluids, we were able to obtain fully saturated samples. However, the design of our NMR cells made it extremely difficult to fully saturate the samples with BEHP without damaging the cell, due to the high viscosity of the fluid. The level of saturation with BEHP ( $\mathrm{S}_{\mathrm{BEHP}}$ ) is used to refer to the volume fraction of the pore space occupied by the fluid, and was determined knowing the initial volume of the fluid added to the NMR cell and the measured porosity. For samples

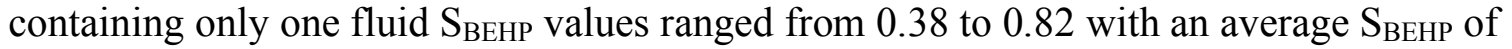
0.57. We prepared two samples with the same sand-clay proportions and made triplicate measurements on each sample. This yielded an estimate of $T_{2}$ measurement uncertainty of $\sim 7 \%$.

To illustrate the form of the data, we show in Figure 1 the distribution of relaxation times obtained for the untreated sand-clay sample with $25 \%$ clay, saturated 
with water. Note that the relaxation time distribution shows a relatively narrow range of pore sizes, indicating that the sample of unconsolidated sand and clay is well mixed. In Figure 2 (a to d) we present the $T_{2} \mathrm{ML}$ values, as a function of clay content (\% by weight), determined from measurements on the four sets of samples (the untreated, A, B, and C sand-clay mixtures) containing only water, toluene or BEHP.

Let us first describe the trends in the data, which provide insight into the observed differences in the $T_{2 \mathrm{ML}}$ values for water, toluene and BEHP. In all types of mixtures, there is a general decrease in $T_{2} \mathrm{ML}$ water and $T_{2 \mathrm{ML}}$ toluene as clay content increases. This is due to the contribution from surface relaxation, which has a relaxation time constant that decreases with increasing $S / V_{p}$ or clay content. (Given the negligible surface area of the sand, compared to that of the clay, $S / V_{p}$ reflects the amount of clay in a sample.) There is also an overall decrease in $T_{2} \mathrm{ML}$ water and $T_{2 \mathrm{ML}}$ toluene as the iron content of the clay in the samples increases in going from the untreated mixtures, to the A, B, then C mixtures. This, again, is due to the contribution from the surface relaxation with a time constant that is known to decrease with an increase in iron content. Given the small number of data points, we cannot make any conclusive observations about trends in $T_{2}$ ML BEHP with iron content; but the data do suggest the same decrease with increasing clay content as seen in the water and toluene data.

Let us now consider the relative magnitudes of the $T_{2 \mathrm{ML}}$ values. The trends in the data indicate that surface relaxation has a significant effect on the relative magnitude of $T_{2 \mathrm{ML}}$ for the two contaminants and water. However, Figure 2 shows that the changes in $T_{2 \mathrm{ML}}$, due to changes in clay and iron content, were much greater for water than the organic fluids. The strength of the surface relaxation, and corresponding magnitude of 
the time constant $T_{2 \mathrm{~S}}$, depends on both the composition of the solid and the solid-fluid interaction. The fact that water is much more strongly affected by surface relaxation than toluene affirms the water-wet nature of the solid surfaces. In the samples containing only toluene or BEHP there is still some interaction with the iron on the surface of the grains but the water-wet nature of the surfaces inhibits the interaction [Bryar and Knight, 2003 a]. Because water has a greater affinity for the sand and clay surfaces than either toluene or BEHP, $T_{2 \mathrm{~S}}$ for water was much less than that for toluene or BEHP.

In all samples with low iron content and low clay content, $T_{2}$ ML of BEHP is less than that of water, due primarily to its very small bulk relaxation time $T_{2 \mathrm{~b}}$. But as the surface relaxation of the water increases due to increasing clay content and/or increasing iron content $T_{2}$ ML of water decreases significantly so that it approaches and, in some cases, becomes less than that of BEHP. In contrast, $T_{2 \mathrm{ML}}$ of toluene is always greater than that of water. While $T_{2 \mathrm{~b}}$ of toluene is less than that of water, the surface relaxation is much weaker in a sand-clay mixture containing toluene than in the same material containing water.

The question that we now address is whether the differences between the response of organic contaminants and that of water are sufficient to allow us to quantify the contaminants in the presence of water. It is important to note that we have seen the difference in the response of the three liquids to be affected by the composition of the material. We thus expect both clay content and iron content to be important factors in determining our ability to use NMR for contaminant quantification. 


\section{SAMPLES CONTAINING TWO FLUIDS}

In each of the experiments on single fluids in the sand-clay samples, a $T_{2}$ distribution was obtained of the form shown in Figure 1, and described in terms of a $T_{2 \mathrm{ML}}$ value. In order to be able to use an NMR relaxation measurement to quantify an immiscible H-containing contaminant co-existing with water in the pore space of a geological material, the NMR inversion process must be able to resolve two distinct $T_{2}$ distributions, one corresponding to water with a $T_{2 \mathrm{ML}}$ value expected to be close to that of water when present as a single fluid, and one corresponding to the contaminant with a $T_{2 \mathrm{ML}}$ value expected to be close to that of the contaminant when present in the material as a single fluid. If two peaks are not seen (there is a single rather than bimodal $T_{2}$ distribution) it means that the $T_{2 \mathrm{ML}}$ values for the fluids are so close that it will not be possible to resolve the NMR contributions from the two fluids and the contaminant cannot be quantified.

In our earlier experiments with simulated NMR relaxation data [Bryar and Knight, 2003 b] we found that the optimal situation for resolving two separate peaks in the $T_{2}$ distribution is one where the water and contaminant contribute equally to the signal intensity; i.e., the sample contains equal moles of water and contaminant $\mathrm{H}$ nuclei. Therefore, our strategy with these laboratory NMR experiments was to start with samples containing roughly equal amounts of water and the contaminant in each sand-clay mixture. If we found that the NMR inversion process was able to recover a bimodal $T_{2}$ distribution, with peaks corresponding to the $T_{2}$ ML values of the single-fluid NMR data, 
we proceeded with further experiments to assess the accuracy with which we could quantify the amount of contaminant.

To prepare a sample for the NMR experiment, accurately weighed amounts of the immiscible pore fluids (water and toluene, or water and BEHP) were first added to a Teflon NMR cell, followed by the solid phase; the sample was compressed and the lid screwed shut. The porosity of each prepared NMR sample was calculated in the same manner as with the single-fluid samples. The total saturation of the sample, which ranged from $0.45-0.89$, and the level of saturation with water $\left(S_{\text {water }}\right)$, toluene $\left(S_{\text {toluene }}\right)$ and BEHP $\left(\mathrm{S}_{\mathrm{BEHP}}\right)$ were calculated from the densities of each fluid and the porosity. With the method of sample preparation, there is no control over the pore-scale location of the fluids. However, we expect the solid surfaces to remain water-wet.

\section{Can We Resolve Water and Toluene in Sand-Clay Mixtures?}

Relaxation time distributions were obtained for each sand-clay mixture containing approximately equal weights of water and toluene. The first question we addressed: Can we resolve the individual NMR response of water and toluene from the NMR data? In most cases the answer to this was, 'Yes.' Nineteen of the 24 relaxation time distributions were bimodal, with the distribution centered at shorter times interpreted as corresponding primarily to the presence of water, and the one at longer times interpreted as corresponding primarily to the presence of toluene. This interpretation is based on our observations in the single-fluid systems where $T_{2} \mathrm{ML}$ toluene was always greater than $T_{2} \mathrm{ML}$ water (see Figure 2) due to the enhanced surface relaxation in the systems containing water. Given the natural water-wet nature of sands and clays, there is no reason to expect that $T_{2}$ 
ML water would ever be greater than $T_{2}$ ML toluene, when these two fluids co-exist in a sample. We expect the water to preferentially occupy the higher $S / V_{p}$ regions of the pore space, either filling pores or coating the solid surfaces. This would shift $T_{2 \mathrm{ML}}$ water to shorter times than observed when the sample was fully saturated with water and $T_{2 \mathrm{ML}}$ toluene to longer times than observed when the sample was fully saturated with toluene.

As an example, the distribution for water and toluene in an untreated sand-clay mixture with $25 \%$ clay is shown in Figure 3. The total saturation of this sample is 0.85 $\left(S_{\text {water }}=0.36, S_{\text {toluene }}=0.49\right)$. The peaks are broad enough that they overlap, but the peak maxima are well separated with $T_{2 \mathrm{ML}}$ values of $0.038 \mathrm{~s}$ (interpreted as water) and $0.62 \mathrm{~s}$ (interpreted as toluene). For comparison, the data for the single fluids in this same material yielded $T_{2 \mathrm{ML} \text { water }}=0.079 \mathrm{~s}$ and $T_{2 \mathrm{ML} \text { toluene }}=0.35$. The changes in the relaxation times of the fluids are consistent with our expected changes in the pore-scale locations of the fluids that would occur in going from a sample fully saturated with one fluid, to the same sample containing two fluids. Some of the change in the $T_{2 \mathrm{ML}}$ values of water and toluene could be a result of bias in the inversion process that seeks to separate the two peaks. This effect was observed with simulated NMR data with known $T_{2}$ values [Bryar and Knight, 2003 b].

While we were able to resolve two distributions in most of the samples, there were some samples where we obtained a single distribution representing the combined signal from water and toluene. The ability to resolve an NMR signal for each of the two fluids, co-existing in a porous medium, clearly depends upon the difference in the $T_{2}$ distributions for the fluids. We expressed this difference as the ratio $T_{2 \mathrm{ML}}$ toluene $/ T_{2 \mathrm{ML}}$ water; all values are presented in Table 2 for samples saturated with water and toluene 
when present as single fluids and as a two-fluid mixture. In the case of water and toluene, we found that a good predictor of the ability-to-resolve a bimodal $T_{2}$ distribution for any given two-fluid sand-clay sample is the magnitude of $T_{2} \mathrm{ML}$ toluene $/ T_{2} \mathrm{ML}$ water for the single-fluid samples. As shown in Table 2, when $T_{2}$ ML toluene $/ T_{2}$ ML water was greater than $\sim 4$, it was always possible to obtain a bimodal distribution, with $T_{2}$ ML values close to those expected for toluene and water. When $T_{2 \mathrm{ML}}$ toluene $/ T_{2} \mathrm{ML}$ water for the single fluids was less than $\sim 3$, a bimodal distribution could not be resolved, denoted by "NR" in the table.

The ratios $T_{2} \mathrm{ML}$ toluene $/ T_{2} \mathrm{ML}$ water when the pore fluid was a two-fluid mixture of toluene and water were calculated assuming that the peak at earlier times corresponds to the toluene signal and the peak at later times corresponds to the water signal. The twofluid ratios $T_{2}$ ML toluene $/ T_{2}$ ML water of the bimodal distributions range from 10 to 97 , indicating that the peaks in the $T_{2}$ distributions were well separated, by approximately one to two decades on the logarithmic scale.

\section{Can We Resolve Water and BEHP in Sand-Clay Mixtures?}

The $T_{2}$ relaxation time distributions were obtained for all sand-clay mixtures containing approximately equal weights of water and BEHP. The question we addressed: Can we resolve the individual NMR response of water and BEHP from the NMR data? In most cases the answer to this was, 'No'. For example, the distribution for water and BEHP in an untreated sand-clay mixture with $25 \%$ clay is shown in Figure 4 . The total saturation of the sample was 0.74 with $\mathrm{S}_{\mathrm{water}}=0.36$ and $\mathrm{S}_{\mathrm{BEHP}}=0.38$. Even though two 
immiscible fluids were present, only one peak is visible. The BEHP signal cannot be resolved from that of water in this medium.

There were some sand-clay mixtures in which BEHP and water could be resolved. In those cases, the peaks were assigned as BEHP or water by comparing the relaxation times with those observed for the single fluids in the same type of sample. As an example, the distribution for water and BEHP in $100 \%$ clay B is shown in Figure 5. The total saturation of this sample is $0.45\left(\mathrm{~S}_{\mathrm{water}}=0.21, \mathrm{~S}_{\mathrm{BEHP}}=0.24\right)$. The peaks overlap slightly, but the peak maxima are well separated with $T_{2} \mathrm{ML}$ values of $0.0063 \mathrm{~s}$ (interpreted as water) and $0.062 \mathrm{~s}$ (interpreted as BEHP). For comparison, the data for the single fluids in this same material yielded $T_{2 \mathrm{ML} \text { water }}=0.0055 \mathrm{~s}$ and $T_{2 \mathrm{MLBEHP}}=0.033$ s. As with the toluene-water results, some of the change in the $T_{2 \mathrm{ML}}$ values of BEHP and water is likely a result of bias in the inversion process [Bryar and Knight, 2003 b]; but a change in pore-scale location of the fluids could also affect the $T_{2}$ ML values.

In Table 3 are given the ratios of the $T_{2}$ ML values for BEHP and water. Unlike the toluene data where, for all samples (single or mixed fluids), $T_{2} \mathrm{ML}$ toluene $>T_{2} \mathrm{ML}$ water the single-fluid NMR data for BEHP yielded $T_{2} \mathrm{ML} \mathrm{BEHP}<T_{2}$ ML water in all untreated samples and all sands and $T_{2 \mathrm{MLBEHP}}>T_{2 \mathrm{ML}}$ water in samples of clay A, clay B and clay C (where the surface relaxation of the water was strong enough to compensate for the very low value of $T_{2 \mathrm{~b}}$ for BEHP). The single-fluid ratios in Table 3 were defined to be greater than

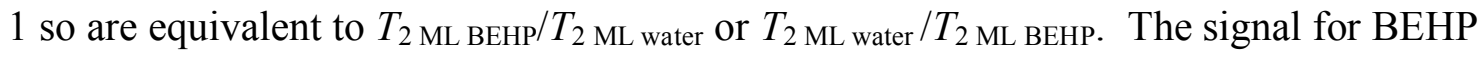
was resolved from that of water for 9 of the 24 sand-clay mixtures; in those cases, the single-fluid $T_{2}$ ratio was 3.4 or greater. In cases when the signal for BEHP was not resolved from that of water, the single-fluid $T_{2}$ ratio was 6.1 or lower. Therefore, single- 
fluid $T_{2 \mathrm{ML}}$ ratios between $\sim 3$ and 7 can not be used to predict whether a separate peak for BEHP will be observed in the $T_{2}$ distribution. Also given in Table 3 are the associated two-fluid ratios obtained from the bimodal relaxation distributions for approximately equal amounts of water and BEHP in the same material. Only three of the samples had a two-fluid $T_{2 \mathrm{ML}}$ ratio greater than 10, indicating that there was considerable overlap of water and BEHP peaks: much more overlap than was typically observed for the samples with toluene and water.

\section{Quantification of Immiscible Toluene and BEHP in the Presence of Water}

The final stage of our study was designed to address the key question that motivated this research: If we can resolve the NMR signal from a contaminant, can we then accurately quantify the amount of contaminant that is present? For any sample where we were able to resolve a bimodal distribution, with two peaks attributable to contaminant and water, it should be possible to calculate the quantity of immiscible contaminant from the amplitude of the $T_{2}$ distribution assigned to the $\operatorname{contaminant}\left(\sum A_{i}\right)_{\text {fluid }}$ :

$$
\left.\operatorname{mass}_{\text {fluid }}=1 / \mathrm{k}^{*}\left(\sum A_{i}\right)_{\text {flud }}\right) / \mathrm{H} \text { density, }
$$

where the $\mathrm{H}$ density is $111.0 \mathrm{mmol} \mathrm{H/g}$ for water, $86.8 \mathrm{mmol} \mathrm{H} / \mathrm{g}$ for toluene, and 97.3 mmol H/g for BEHP; $k$, the amplitude per mmol $\mathrm{H}$ was $260.3 \pm 1.2$ for our NMR instrument with the parameters used for these experiments. For relaxation time distributions with no overlap between the contaminant and water peaks it was 
straightforward to obtain $\left(\sum A_{i}\right)_{\text {fluid }}$ for both contaminant and water. In cases when the peaks overlapped we assumed that the minimum amplitude between the two peaks represented the division between the contaminant and water contributions.

Figure 6 shows the percent difference between the amount of contaminant fluid quantified by NMR using (7) and the amount actually present in the sample. The absolute value of the percent difference is plotted against the two-fluid $T_{2}$ ML ratio obtained from the associated bimodal $T_{2}$ distribution. Overall, there is a reduction in the percent difference (i.e., an improvement in accuracy) with increasing $T_{2} \mathrm{ML}$ ratio. At low values of the $T_{2}$ ML ratio, where the contaminant and water peaks overlap the most, the difference ranges from $\sim 5$ to $\sim 50 \%$. For high values of the $T_{2}$ ML ratio, where the contaminant and water peaks were well-separated, the difference is $1-10 \%$.

For each sand-clay mixture which showed resolved peaks for water and toluene in Table 2 and for water and BEHP in Table 3, additional NMR samples were prepared with smaller amounts of the contaminant. Successive samples were prepared with decreasing amounts of contaminant until the signal from the contaminant could no longer be resolved from the water signal and only one peak remained in the $T_{2}$ distribution. For each sample, the amount of contaminant was quantified from the peak amplitude using (7). Figure 7 displays the amount quantified with NMR versus the amount present. The method appears to have an equal probability of overestimating and underestimating the quantity of contaminant. We found that the accuracy of our estimate did not depend on the amount of contaminant present, provided that two peaks were still visible in the $T_{2}$ distribution. The only factor which affected accuracy (other than the signal-to-noise ratio 
of the raw data) was the extent of overlap between the water and contaminant peaks (as was illustrated in Figure 6).

We assigned the smallest amount of contaminant that we were able to quantify in each sand-clay mixture as the detection limit. Tables 4 and 5 list the detection limits for toluene and BEHP respectively. The detection limits are reported as the weight percent of the pore fluid (water-contaminant mixture) that is toluene or BEHP. The results for toluene are encouraging. The detection limits were under $15 \%$ toluene for 18 of the sandclay mixtures and less than 5\% toluene in 13 sand-clay mixtures. The highest (i.e., worst) detection limit observed was $18 \%$ toluene. The lowest detection limit observed was $3 \%$; this is equivalent to $S_{\text {toluene }}=0.035$ for a fully saturated medium. The detection limits for BEHP were not as good. In three of the sand-clay mixtures, the detection limits were $35 \%$ and $50 \%$. Only 6 of the 24 sand-clay mixtures had detection limits under $15 \%$ BEHP. The detection limit was approximately $5 \%$, equivalent to $\mathrm{S}_{\mathrm{BEHP}}=0.051$ for a fully saturated medium, in 5 of those cases. To achieve the same detection limits in the field would require acquisition of data with signal-to-noise ratio greater than 100 .

\section{CONCLUSIONS}

These results are encouraging for those considering the acquisition of NMR relaxation data for quantifying immiscible organic fluids in groundwater at contaminated sites. Although the sand-clay mixtures used in this study do not represent all possible sediments, both clay- and iron-content were varied to cover a wide range of NMR relaxation behavior, with $T_{2} \mathrm{ML}$ water values from 0.68 to $0.0068 \mathrm{~s}$. Our results should be directly applicable to many near-surface environments. 
Our results suggest that it should be possible to quantify toluene in the presence of water in most sediments, provided some clay is present. Furthermore, the detection limits for toluene are low enough to be useful provided the contaminant is present at more than trace quantities. Detection limits could be improved beyond the 3-18\% levels observed in this study by increasing the signal-to-noise ratio of the data significantly, but the additional time required for data collection might be impractical. Since BEHP could only be distinguished from water in untreated sand and in high-iron clay-rich mixtures, it is unlikely that NMR relaxation measurements of this type would be useful for quantifying BEHP in the presence of water in most contaminated environments.

The results for toluene are applicable to other immiscible contaminants that are relatively non-polar organic liquids with wetting characteristics and viscosities similar to that of toluene. As long as the fluid with those characteristics exhibited a $T_{2} \mathrm{ML}$ value higher than water in the same sediment its NMR signal would likely be resolvable from water to the same extent as toluene. However, there will be a difference in detection limit of the method for other contaminants depending on their $\mathrm{H}$ density (mol H/g contaminant). For example, the results of our study predict that if the detection limit for toluene in a sediment was $5 \%$, benzene and dichloromethane would have detection limits of approximately $6 \%$ and $18 \%$ respectively in the same sediment. Unfortunately, the $\mathrm{H}$ density of highly chlorinated contaminants such as trichloroethene (TCE) are so low that quantifying them with NMR in the presence of water is unlikely unless data with significantly higher signal to noise ratios were acquired.

The main weakness of this method, other than its inability to detect trace levels of contaminants, is that the resolution of the contaminant signal and the accuracy of 
contaminant quantification are both highly dependent on soil lithology and iron content. This suggests that before using NMR at a contaminated site, a series of laboratory NMR measurements should be made using uncontaminated sediments collected at the site to determine if the NMR signal for the contaminant could be resolved from that of water.

The great strength in using NMR for the characterization and remediation of contaminated sites, is its ability to quantify rather than simply detect the presence of immiscible organic contaminants. In this laboratory study we explored the factors controlling the use of NMR to quantify toluene and BEHP in a range of sand-clay mixtures. We are confident that further laboratory studies will show that NMR could be used in a wide range of geologic settings to detect and quantify other organic contaminants. What is now needed is the research and development required to improve NMR field systems so that the successful quantification of contaminants in the laboratory can be duplicated at field sites.

Acknowledgements. This work was initiated with funding to R. Knight under Grant No. DE-FG07-96ER14711 and DE-FG07-00ER15118-A000 Environmental Management Science Program (ESMP), Office of Environment Management, United States Department of Energy (DOE). The work was continued and concluded with funding to R. Knight under Grant DE-FG02-03ER15382-A0003 from the United States Department of Energy. However, any opinions, findings, conclusions or recommendations expressed herein are those of the authors and do not necessarily reflect views of DOE.

\section{REFERENCES}


Abragam, A. (1961), Principles of Nuclear Magnetism, Oxford University Press.

Banavar, J.R. and L.M. Schwartz (1987), Magnetic Resonance as a Probe of Permeability in Porous Media, Phys. Rev. Lett., 58(14), 1411-1414.

Bloembergen, N., E.M. Percell and R.V. Pound (1948), Relaxation effects in nuclear magnetic resonance absorption, Phys. Rev., 3, 679-712.

Brown, R.J.S. and I. Fatt (1956), Measurements of fractional wettability of oilfield rocks by the nuclear magnetic relaxation method, Trans. Am. Inst. Min. Metall. Pet. Eng., 207, 262-264.

Brown, R.J.S. and P. Fantazinni (1993), Conditions for initial quasilinear $\mathrm{T}_{2}^{-1}$ versus $\tau$ for Carr-Purcell-Meiboom-Gill NMR with diffusion and susceptibility differences in porous media and tissues, Phys. Rev. B, 47, 14823-14828.

Brown, R.J.S. and B.W. Gamson (1960), Nuclear magnetism logging, Trans. Am. Inst. Min. Metall. Pet. Eng., 219, 201-219.

Brownstein, K.R. and C.E. Tarr (1979), Importance of classical diffusion in NMR studies of water in biological cells, Phys. Rev., 19, 2446-2453.

Brunauer, S., Emmett, P. H. and E. Teller (1938), Adsorption of gases in multimolecular layers, American Chemical Society Journal, 60, 309-319.

Bryar, T.R., C.J. Daughney and R. J. Knight (2000), Paramagnetic effects of iron(III) species on nuclear magnetic relaxation of fluid protons in porous media, J. Magn. Reson., 142, 74-85. 
Bryar, T.R., R.J. Knight and T.P. Nielsen (2001), Laboratory studies to assess the potential for light non-aqueous liquid detection in contaminated soil with proton nuclear magnetic resonance, EOS Trans. AGU, 82(47), Fall Meet. Suppl.

Bryar, T.R. and R.J Knight (2003 a), Laboratory studies of the detection of sorbed oil with proton NMR, Geophysics, 68, 942-948.

Bryar, T.R. and R.J. Knight (2003 b), Detection limits for immiscible liquid organic contaminants using proton nuclear magnetic resonance, Proc. Symp. Appl. Geophys. Eng. Environ. Problems (SAGEEP), April 6-10, 2003, San Antonio, TX.

Chan, C.Y. and R.J. Knight (1999), Determining water content and saturation from dielectric measurements in layered materials, Water Resour. Res., 35, 85-93.

Flaum, M., J. Chen and G.J. Hirasaki (2005), NMR diffusion-editing for DT2 maps: Application to recognition of wettability change, Petrophysics, 46(2), 113-118.

Foley, I., S.A. Farooqui and R.L. Kleinberg (1996), Effect of paramagnetic ions on NMR relaxation of fluids at solid surfaces, J. Magn. Reson., 123, 95-104.

Grantham, M.C., P.M. Dove and T.J. DiChristina (1997), Microbially catalyzed dissolution of iron and aluminum oxyhyroxide mineral surface coatings, Geochim. Cosmochim. Acta, 61, 4467-4477.

Hedberg, S.A., R.J. Knight, A.L. MacKay and K.P. Whittall (1993), The use of nuclear magnetic resonance for studying and detecting hydrocarbon contaminants in porous rocks, Water Resour. Res., 29, 1163-1170.

Kenyon, W.E. (1992), Nuclear magnetic resonance as a petrophysical measurement, Nucl. Geophys., 6, 153-171. 
Korringa, J. (1956), Interpretation of data on nuclear spin relaxation of liquids for large surface-to-volume ratios, Bull. Am. Phys. Soc., Ser. II, 1, 216.

Legchenko, A., A. Beauce, A. Guillen, P. Valla and J. Bernard (1997), Natural variations in the magnetic resonance signal used in PMR groundwater prospecting from the surface, J. Environ. Eng. Geophys., 2, 173-190.

Li, C., P. Tercier and R. Knight (2001), The effect of sorbed oil on the dielectric properties of sand and clay, Water Resour. Res., 37, 1783-1793.

Lucius, J.E., G.R. Olhoeft, P.L. Hill and S.K. Duke (1992), Properties and Hazards of 108 Selected Substances, U.S. Geological Survey, Open File Report 92-527.

Olhoeft, G.R. (1992), Geophysical detection of hydrocarbon and organic chemical contamination, Proc. Symp. Appl. Geophys. Eng. Environ. Problems (SAGEEP), 2, $587-595$.

Schirov, M., A. Legchenko and G. Creer (1991), New direct non-invasive ground water detection technology for Australia, Expl. Geophys., 22, 333-338.

Senturia, S.D. and J.D. Robinson (1970), Nuclear spin-lattice relaxation of liquids confined in porous solids, Soc. Petrol. Eng. J., 10, 237-244.

Straley, C., C.E. Morriss, W.E. Kenyon and J.J. Howard (1995), NMR in partially saturated rocks: laboratory insights on free fluid index and comparison with borehole logs, Log Analyst, 36, 40-65.

Swoboda-Colberg, N.G. (1995), Chemical contamination of the environment: sources, types and fate of synthetic organic chemicals, in Microbial transformations and degradation of toxic organic chemicals, edited by L.Y. Young and C.E. Cerniglia, pp.27-74, Wiley-Liss, Inc., New York, N.Y. 
Torrey, H.C. (1956), Theory of nuclear spin relaxation of liquids for large surface-tovolume ratios, Bull. Am. Phys. Soc., Ser. II, 1, 216.

Whittall, K.P. and A.L. MacKay (1989), Quantitative interpretation of NMR relaxation data, J. Magn. Reson., 84, 134-152.

Whittall, K.P., (1994) Analysis of large one-dimensional and two-dimensional relaxation data sets, J. Magn. Reson., A110, 214-218. 
Table 1. Measured properties of sands and clays.

\begin{tabular}{|c|c|c|c|c|c|c|c|c|}
\hline \multirow[b]{2}{*}{ Material properties } & \multicolumn{2}{|c|}{$\begin{array}{l}\text { Untreated } \\
\text { Materials }\end{array}$} & \multicolumn{3}{|c|}{ FeOOH Coated Sands } & \multicolumn{3}{|c|}{ FeOOH Coated Clays } \\
\hline & Sand & Clay & Sand A & Sand B & Sand C & Clay A & Clay B & Clay C \\
\hline Grain Density, g/cc & 2.67 & 2.62 & 2.67 & 2.67 & 2.67 & 2.62 & 2.62 & 2.62 \\
\hline Iron Content, $\% \mathrm{Fe}$ & 0.34 & 0.1 & 0.4 & 0.35 & 0.94 & 0.2 & 0.31 & 0.44 \\
\hline Specific Surface Area, $\mathrm{m}^{2} / \mathrm{g}$ & 0.032 & 12.44 & 0.034 & 0.026 & 0.038 & 10.5 & 9.9 & 9.35 \\
\hline
\end{tabular}


Table 2. Ratios of the $T_{2}$ mL values for toluene and water in each sand-clay mixture. "Single" refers to the ratios obtained when the samples contained a single fluid. "Mixture" refers to the ratio of the $T_{2}$ ML values for each peak in the bimodal $T_{2}$ distribution obtained from a $\sim 50 / 50$ (by weight) mixture of toluene and water; NR indicates that a bimodal distribution was not resolved.

\begin{tabular}{|c|c|c|c|c|c|c|c|c|}
\hline \multirow[b]{2}{*}{$\begin{array}{l}\text { Clay Content } \\
\text { (\% by weight) }\end{array}$} & \multicolumn{2}{|c|}{$\begin{array}{c}\text { Untreated } \\
\text { Sand-Clay Mixtures }\end{array}$} & \multicolumn{2}{|c|}{$\begin{array}{c}\text { A } \\
\text { Sand-Clay Mixtures }\end{array}$} & \multicolumn{2}{|c|}{$\begin{array}{c}\text { B } \\
\text { Sand-Clay Mixtures }\end{array}$} & \multicolumn{2}{|c|}{$\begin{array}{c}\mathrm{C} \\
\text { Sand-Clay Mixtures }\end{array}$} \\
\hline & Single & Mixture & Single & Mixture & Single & Mixture & Single & Mixture \\
\hline 0 & 1.3 & NR & 2.6 & NR & 2.9 & NR & 7.9 & 17 \\
\hline 5 & 1.9 & NR & 5.0 & 23 & 6.4 & 27 & 22 & 57 \\
\hline 10 & 2.4 & NR & 6.3 & 27 & 13 & 43 & 37 & 97 \\
\hline 25 & 4.4 & 16 & 17 & 29 & 19 & 54 & 59 & 95 \\
\hline 50 & 7.0 & 10 & 16 & 38 & 26 & 71 & 60 & 96 \\
\hline 100 & 6.4 & 17 & 20 & 37 & 31 & 43 & 63 & 95 \\
\hline
\end{tabular}


Table 3. Ratios of the $T_{2 \mathrm{ML}}$ values for BEHP and water in each sand-clay mixture. "Single" refers to the ratios obtained when the samples contained a single fluid. "Mixture" refers to the ratio of the $T_{2} \mathrm{ML}$ values for each peak in the bimodal $T_{2}$ distribution obtained from a $~ 50 / 50$ (by weight) mixture of BEHP and water; NR indicates that a bimodal distribution was not resolved. Empty cells are those for which single-fluid measurements with BEHP were not made.

\begin{tabular}{|c|c|c|c|c|c|c|c|c|}
\hline \multirow[b]{2}{*}{$\begin{array}{l}\text { Clay Content } \\
\text { (\% by weight) }\end{array}$} & \multicolumn{2}{|c|}{$\begin{array}{c}\text { Untreated } \\
\text { Sand-Clay Mixtures }\end{array}$} & \multicolumn{2}{|c|}{$\begin{array}{c}\text { A } \\
\text { Sand-Clay Mixtures }\end{array}$} & \multicolumn{2}{|c|}{$\begin{array}{c}\text { B } \\
\text { Sand-Clay Mixtures }\end{array}$} & \multicolumn{2}{|c|}{$\begin{array}{c}\text { C } \\
\text { Sand-Clay Mixtures }\end{array}$} \\
\hline & Single & Mixture & Single & Mixture & Single & Mixture & Single & Mixture \\
\hline 0 & 10 & 13 & 4.4 & NR & 4.7 & 7.7 & 1.7 & NR \\
\hline 5 & 6.1 & NR & -- & NR & -- & NR & -- & NR \\
\hline 10 & 3.0 & NR & -- & NR & -- & NR & -- & 6.3 \\
\hline 25 & 1.6 & NR & -- & NR & -- & NR & -- & 8.5 \\
\hline 50 & 1.6 & NR & -- & NR & -- & 7.7 & -- & 13 \\
\hline 100 & 1.5 & NR & 3.4 & 8.6 & 6.0 & 9.7 & 13 & 20 \\
\hline
\end{tabular}


Table 4. The detection limit for toluene is listed as the weight percent of the pore fluid (watertoluene mixture) that is toluene. No detection limit is reported if the contaminant signal was not resolved from that of water.

\begin{tabular}{ccccc}
\hline $\begin{array}{c}\text { Clay Content } \\
\text { \% by weight) }\end{array}$ & $\begin{array}{c}\text { Untreated } \\
\text { Sand-Clay } \\
\text { Mixtures }\end{array}$ & $\begin{array}{c}\text { A } \\
\text { Sand-Clay } \\
\text { Mixtures }\end{array}$ & $\begin{array}{c}\text { B } \\
\text { Sand-Clay } \\
\text { Mixtures }\end{array}$ & $\begin{array}{c}\text { C } \\
\text { Sand-Clay } \\
\text { Mixtures }\end{array}$ \\
\hline 0 & ----- & ----- & ----- & 9.9 \\
5 & ----- & 14 & 18 & 3.0 \\
10 & ---- & 10 & 8.8 & 3.1 \\
25 & 15 & 3.9 & 3.2 & 3.0 \\
50 & 4.3 & 3.0 & 3.4 & 3.0 \\
100 & 3.0 & 3.0 & 3.0 & 3.1 \\
\hline
\end{tabular}


Table 5. The detection limit for BEHP is listed as the weight percent of the pore fluid (water-BEHP mixture) that is BEHP. No detection limit is reported if the contaminant signal was not resolved from that of water.

\begin{tabular}{ccccc}
\hline $\begin{array}{c}\text { Clay Content } \\
\text { (\% by weight) }\end{array}$ & $\begin{array}{c}\text { Untreated } \\
\text { Sand-Clay } \\
\text { Mixtures }\end{array}$ & $\begin{array}{c}\text { A } \\
\text { Sand-Clay } \\
\text { Mixtures }\end{array}$ & $\begin{array}{c}\text { B } \\
\text { Sand-Clay } \\
\text { Mixtures }\end{array}$ & $\begin{array}{c}\text { C } \\
\text { Sand-Clay } \\
\text { Mixtures }\end{array}$ \\
\hline 0 & 4.2 & ----- & 50 & ----- \\
5 & ---- & ---- & ---- & ---- \\
10 & ---- & ---- & ---- & 35 \\
25 & ---- & ---- & --- & 5.1 \\
50 & ---- & --- & 35 & 5.1 \\
100 & ---- & 9.8 & 5.1 & 4.7 \\
\hline
\end{tabular}




\section{FIGURES}

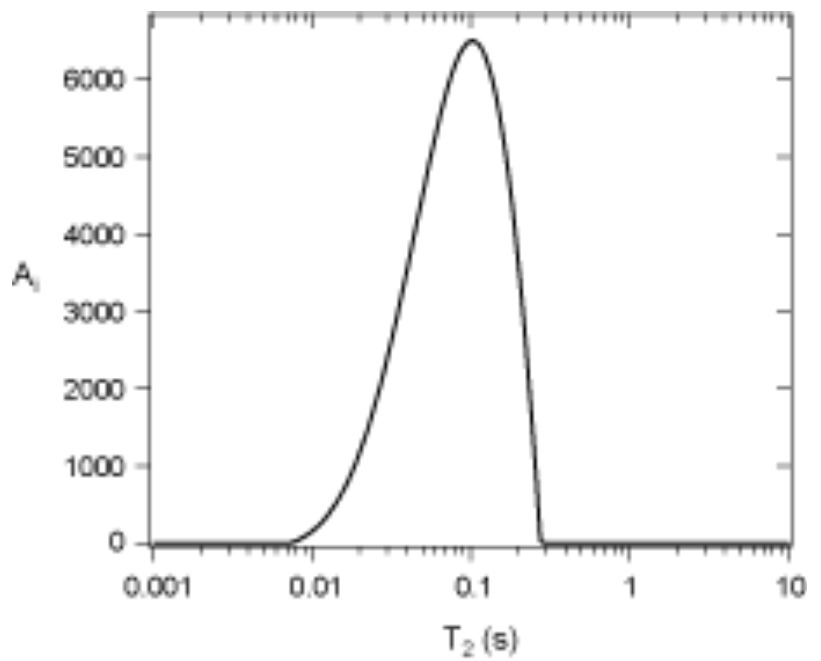

Figure 1. $T_{2}$ relaxation time distribution obtained for the untreated sand-clay sample with $25 \%$ clay, saturated with water. 

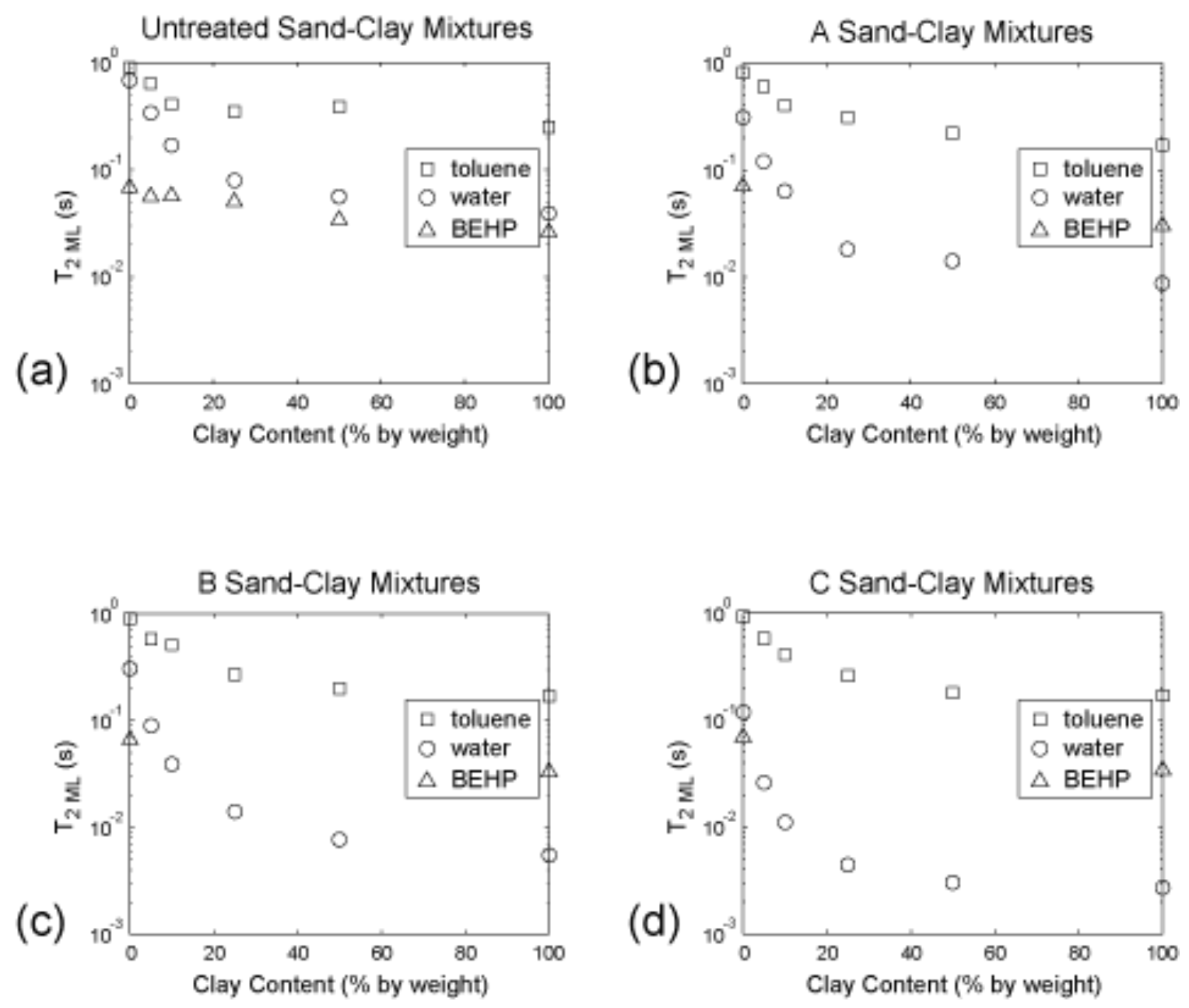

Figure 2. $T_{2 \mathrm{ML}}$ values, as a function of clay content ( $\%$ by weight), determined from measurements on the four sets of samples (the untreated, A, B, and C sand-clay mixtures) containing only water, toluene or BEHP. 


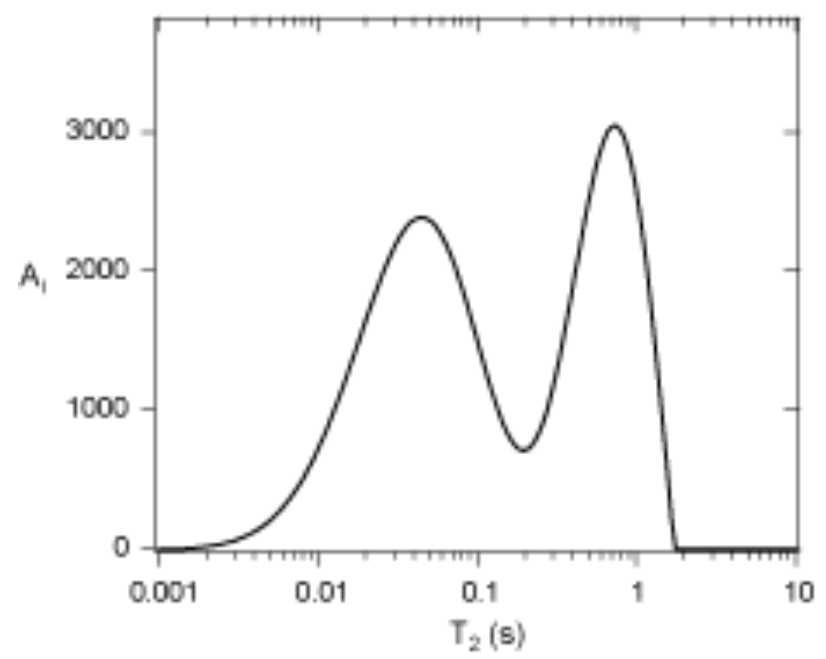

Figure 3. $T_{2}$ relaxation time distribution obtained for water and toluene $\left(\mathrm{S}_{\mathrm{water}}=0.36\right.$, $\left.S_{\text {toluene }}=0.49\right)$ in an untreated sand-clay mixture with $25 \%$ clay.

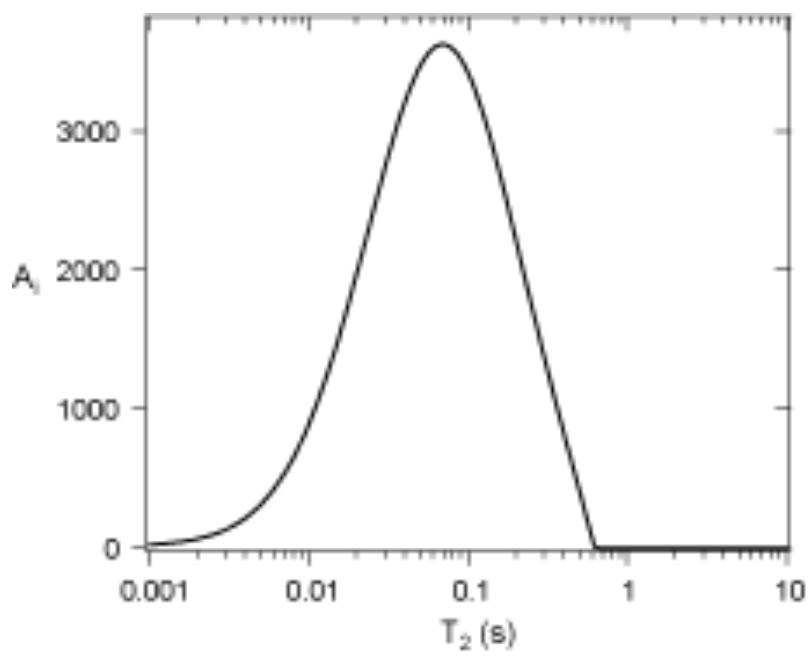

Figure 4. $T_{2}$ relaxation time distribution obtained for water and $\operatorname{BEHP}\left(\mathrm{S}_{\text {water }}=0.36, \mathrm{~S}_{\mathrm{BEHP}}\right.$ $=0.38$ ) in an untreated sand-clay mixture with $25 \%$ clay. 


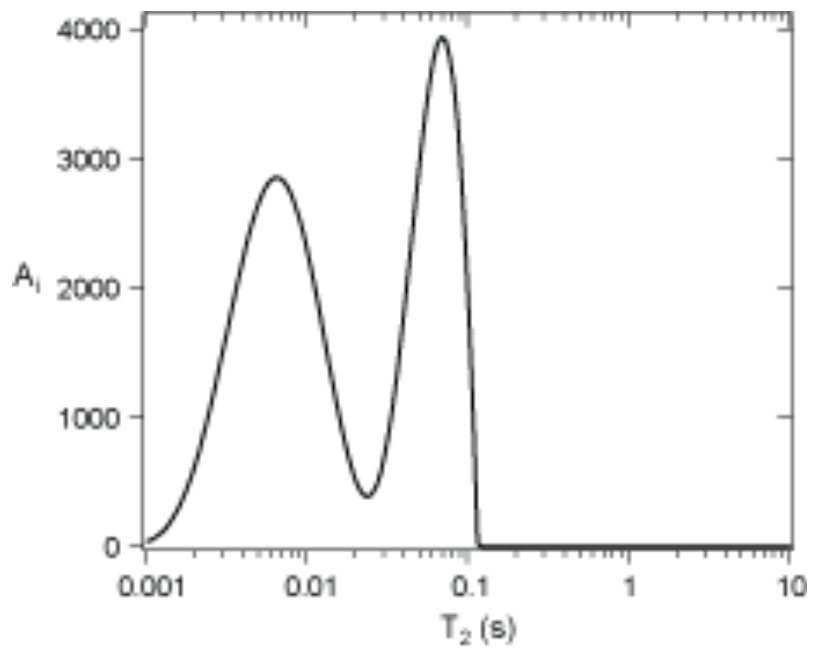

Figure 5. $T_{2}$ relaxation time distribution obtained for water and BEHP $\left(\mathrm{S}_{\text {water }}=0.21, \mathrm{~S}_{\mathrm{BEHP}}\right.$ $=0.24)$ in $100 \%$ clay $\mathrm{B}$.

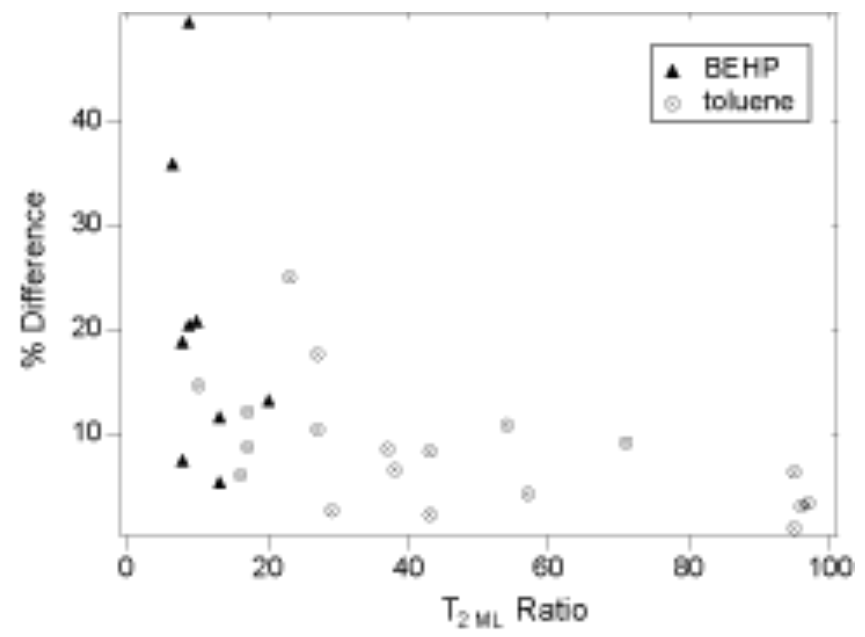

Figure 6. Absolute value of the percent difference between the amount of contaminant detected by NMR and the amount present, plotted against the two-fluid $T_{2 \mathrm{ML}}$ ratio for every sand-clay mixture which had a bimodal $T_{2}$ distribution. All samples contained roughly equal quantities of water and contaminant. 


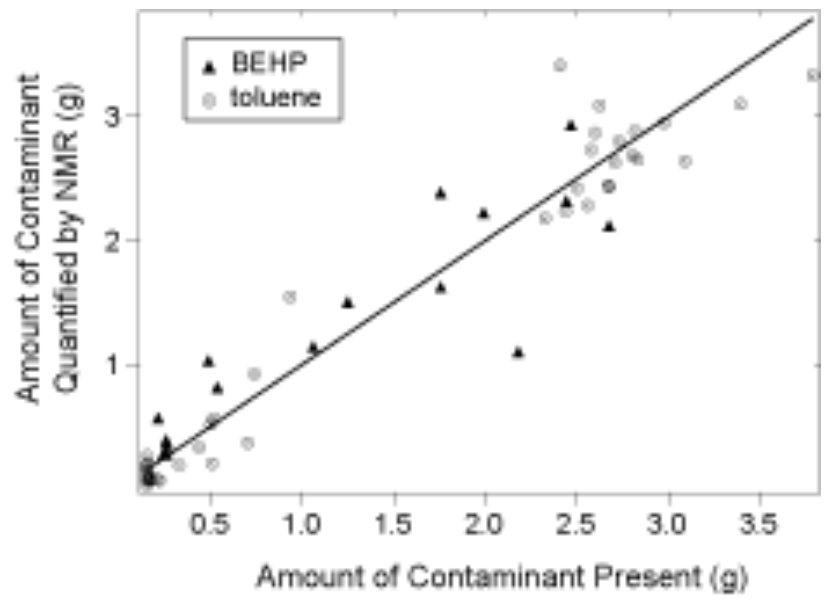

Figure 7. Mass of contaminant quantified by NMR as a function of mass of contaminant present, for all samples which had a bimodal $T_{2}$ distribution. 


\title{
A laboratory study to determine the effect of iron oxides on proton NMR measurements
}

\author{
Kristina Keating ${ }^{1}$ and Rosemary Knight ${ }^{1}$
}

\begin{abstract}
Using laboratory methods, we investigate the effect of the presence and mineralogic form of iron on measured proton nuclear magnetic resonance (NMR) relaxation rates. Five samples of quartz sand were coated with ferrihydrite, goethite, hematite, lepidocrocite, and magnetite. The relaxation rates for these iron-oxide-coated sands saturated with water were measured and compared to the relaxation rate of quartz sand saturated with water. We found that the presence of the iron oxides led to increases in the relaxation rates by increasing the surface relaxation rate. The magnitude of the surface relaxation rate was different for the various iron-oxide minerals because of changes in both the surface-area-to-volume ratio of the pore space, and the surface relaxivity. The relaxation rate of the magnetite-coated sand was further increased because of internal magnetic field gradients caused by the presence of magnetite. We conclude that both the concentration and mineralogical form of iron can have a significant impact on NMR relaxation behavior.
\end{abstract}

\section{INTRODUCTION}

Proton nuclear magnetic resonance (NMR) has been widely used in the geophysical and medical communities to detect the presence of hydrogen nuclei $\left({ }^{1} \mathrm{H}\right)$ and determine their physiochemical environment. NMR measurements in the earth sciences can be made in the laboratory, or in the field using a well-logging device or a system deployed at the earth's surface. NMR logging has been employed in the oil industry to quantify water and hydrocarbon content and to estimate pore size and permeability (e.g., Seevers, 1966; Timur, 1969; Korb et al., 2003). The surface-based system, referred to as MRS (magnetic resonance sounding), has been used to estimate the water content and the permeability of the top $100 \mathrm{~m}$ of earth (e.g., Shusha- kov, 1996; Legchenko et al., 2002). The use of NMR to determine the permeability of water-saturated geologic materials is the application of interest in our research.

If we consider a water-saturated geologic material, the NMR experiment (in the laboratory or in the field) consists of measuring the rate at which the bulk nuclear magnetization of the water within the sampled volume of material returns (relaxes) to equilibrium after being perturbed by a radio-frequency pulse. The measured relaxation rate is related to the surface-area-to-volume ratio of the water-filled pore space; this is the link that allows the use of NMR data to estimate pore sizes (e.g., Timur, 1969; Yaramanci et al., 2002) and permeability (e.g., Vogeley and Moses, 1992; Legchenko et al., 2002).

A complicating factor in the interpretation of NMR relaxation rates is the effect of $\mathrm{Fe}(\mathrm{III})$, a paramagnetic species commonly found in geologic material. Previous laboratory studies (Foley et al., 1996; Bryar et al., 2000) have shown conclusively that an increase in the concentration of $\mathrm{Fe}(\mathrm{III})$, in the solid phase of a geologic material, will cause an increase in the NMR relaxation rate. What has not been investigated, however, is the role of the mineralogic form of the $\mathrm{Fe}(\mathrm{III})$. That is, is it simply the concentration of Fe(III) that determines the effect of $\mathrm{Fe}$ (III) on NMR data, or must the mineralogic form of $\mathrm{Fe}$ (III) also be considered?

Publications in the medical literature clearly show that the chemical form of iron affects proton NMR relaxation rates (Yilmaz et al., 1990; Babes et al., 1998; Gossuin et al., 2002). It has been suggested, based on theoretical modeling, that differences in relaxation rates resulting from changes in the form of the iron can be attributed to the size of the particles containing the paramagnetic species, the distance of the paramagnetic species from the relaxing protons, and the distribution and concentration of the paramagnetic species (Gillis and Koenig, 1987). These results and discussion in the medical literature led us to hypothesize that the mineralogic form of Fe(III) would affect the NMR relaxation rates of water-saturated geologic materials.

Although iron can be found in many forms, we limited this study to iron-oxide minerals. We compared NMR relaxation rates for water in sands coated with known concentrations of $\mathrm{Fe}(\mathrm{III})$ in the form

Manuscript received by the Editor March 23, 2006; revised manuscript received September 2, 2006; published online December 29, 2006.

${ }^{1}$ Stanford University, Department of Geophysics, Mitchell Building, Stanford, California 94304. E-mail: kkeat@pangea.stanford.edu; rknight@ pangea.stanford.edu.

(C) 2007 Society of Exploration Geophysicists. All rights reserved. 
of ferrihydrite $\left[\mathrm{Fe}(\mathrm{OH})_{3} \cdot \mathrm{nH}_{2} \mathrm{O}\right]$, goethite $(\alpha-\mathrm{FeOOH})$, hematite $\left(\alpha-\mathrm{Fe}_{2} \mathrm{O}_{3}\right)$, lepidocrocite $(\gamma-\mathrm{FeOOH})$, and magnetite $\left(\mathrm{Fe}_{3} \mathrm{O}_{4}\right)$. These iron oxides were chosen to represent a variety of $\mathrm{Fe}(\mathrm{III})$-bearing, naturally occurring iron minerals. The selected iron oxides are pure $\mathrm{Fe}(\mathrm{III})$ minerals, with the exception of magnetite which contains both $\mathrm{Fe}(\mathrm{III})$ and $\mathrm{Fe}(\mathrm{II})$. Understanding the effect of these iron minerals is essential if we are to use NMR data, in the laboratory or the field, to obtain accurate information about the permeability of geologic materials.

\section{NMR RELAXATION THEORY}

All atoms with an odd number of protons or neutrons possess a nuclear spin angular momentum. In many geologic applications, the hydrogen atom $\left({ }^{1} \mathrm{H}\right)$ with a single proton is of interest because of its presence in water. In a static magnetic field $\left(B_{0}\right)$, the nuclear spins in the water align with the field, resulting in a net magnetization $\left(M_{0}\right)$ which is proportional to the number of ${ }^{1} \mathrm{H}$ in the sample. $M_{0}$ processes at the Larmor frequency $f_{0}$, which is related to $B_{0}$ by

$$
f_{0}=\frac{1}{2 \pi} \gamma\left|B_{0}\right|
$$

where $\gamma$ is the gyromagnetic ratio for hydrogen protons in water molecules $[\gamma=0.267 \mathrm{rad} /(\mathrm{nT} \cdot \mathrm{s})]$. For MRS instruments, $f_{0}$ ranges from 0.8 to $2.8 \mathrm{kHz}$; for NMR well-logging instruments, $f_{0}$ ranges from 0.5 to $2 \mathrm{MHz}$; and for laboratory instruments, $f_{0}$ ranges from 0.01 to $900 \mathrm{MHz}$. If a magnetic field oscillating at $f_{0}$ is applied for a short time, the nuclear spins move away from, and then relax to, their equilibrium position. This results in a measurable signal from the bulk nuclear magnetization $(M)$, which can be described in terms of a transverse magnetization $M_{x y}$, and a longitudinal magnetization $M_{z}$. Parameters that describe the observed relaxation of $M_{x y}$ are denoted with the subscript 2 , and those describing the observed relaxation of $M_{z}$ are denoted with the subscript 1 . In this study we measured $M_{x y}$, which is the parameter detected by most well-logging and surface-based NMR instruments.

For bulk fluids, the return or relaxation to equilibrium over time $(t)$ behaves as an exponential decay:

$$
M_{x y}(t)=M_{0}\left(e^{-t / T_{2 B}}\right),
$$

where $T_{2 B}$ is the bulk fluid relaxation time; the inverse, $T_{2 B}^{-1}$, is referred to as the bulk fluid relaxation rate and results from dipole-dipole molecular interactions. The magnitude of $T_{2 B}^{-1}$ for a fluid depends on the viscosity via the reduction of rotational mobility, the concentration of dissolved paramagnetic species such as dissolved oxygen, $\mathrm{Mn}^{2+}$ ions, or $\mathrm{Fe}^{3+}$ ions, and $\mathrm{pH}$ (Bloembergen et al., 1948; Bryar et al., 2000).

The relaxation rate of water in a porous material is generally found to be greater than $T_{2 B}^{-1}$ because of two other mechanisms that can enhance relaxation. The relaxation rate of water in a pore $T_{2}^{-1}$ (where $T_{2}$ is the relaxation time), is described as a sum of three relaxation rates (Brownstein and Tarr, 1979):

$$
T_{2}^{-1}=T_{2 B}^{-1}+T_{2 s}^{-1}+T_{2 D}^{-1},
$$

where $T_{2 S}^{-1}$ is the surface relaxation rate and $T_{2 D}^{-1}$ is the diffusion relaxation rate $\left(T_{2 S}\right.$ and $T_{2 D}$ are the surface and diffusion relaxation times.) The surface relaxation rate is determined by the interaction that occurs between the water protons and paramagnetic sites on the solid surface of the pore space. In the case of fast diffusion, which as- sumes that all protons travel to and relax at the solid surface in the time interval of the NMR experiment, the surface relaxation rate is given by (Senturia and Robinson, 1970; Brownstein and Tarr, 1979)

$$
T_{2 s}^{-1}=\rho_{2} \frac{S}{V},
$$

where $S / V$ is the surface-area-to-volume ratio of the water-filled pore and $\rho_{2}$ is the surface relaxivity. The diffusion relaxation rate is determined by the effect of the magnetic properties of the solid on the diffusing water molecules. The diffusion relaxation rate $T_{2 D}^{-1}$ is related to the average internal gradient of the magnetic field $(G)$ and the diffusion coefficient of water $(D)$ by

$$
T_{2 D}^{-1}=\frac{D}{12}\left(\gamma G t_{E}\right)^{2},
$$

where $\gamma$ is the gyromagnetic ratio, and $t_{E}$ is the echo time-a rephasing parameter used during the Carr-Purcell-Meiboom-Gill (CPMG) pulse sequence. The CPMG pulse sequence (Carr and Purcell, 1954; Meiboom and Gill, 1958) was developed to rephase proton spins in a solid in the presence of nonuniform magnetic fields. The average internal gradient of the magnetic field is caused by the magnetic susceptibility of each phase in the geologic material, and by the differences in susceptibility between the phases. Ferrihydrite, goethite, hematite, and lepidocrocite are antiferromagnetic minerals with magnetic susceptibilities ranging from $0.5 \times 10^{-3}$ to $40 \times 10^{-3} \mathrm{SI}$. Magnetate is a ferrimagnetic mineral with a magnetic susceptibility ranging from $1000 \times 10^{-3}$ to $5700 \times 10^{-3}$ SI. Quartz and water are both diamagnetic, with the magnetic susceptibility of quartz ranging from $-13 \times 10^{-6}$ to $-17 \times 10^{-6} \mathrm{SI}$, and the magnetic susceptibility of water equal to $-9 \times 10^{-6}$ SI (Hunt et al., 1995; Cornell and Schwertmann, 2003)

When water saturates a geological material with a range of pore sizes, a multiexponential decay is observed,

$$
M(t)=\sum_{i} m_{i} e^{-t / T_{2 i}},
$$

where $m_{i}$ is proportional to the number of moles of ${ }^{1} \mathrm{H}$ relaxing with rate $T_{2 i}^{-1}$ and $M(0)$ is proportional to the total moles of ${ }^{1} \mathrm{H}$. The values of $m_{i}$ versus $T_{2 i}$ are often plotted to show the distribution of relaxation times. In studies of the NMR response of porous materials, the arithmetic mean of $\log T_{2}\left(T_{2 M L}\right)$ is typically calculated from the distribution of relaxation times and used to represent the relaxation behavior. Equation 3 then becomes

$$
T_{2 M L}^{-1}=T_{2 B}^{-1}+T_{2 s}^{-1}+T_{2 D}^{-1},
$$

where $T_{2 B}^{-1}, T_{2 s}^{-1}, T_{2 D}^{-1}$ are now taken to be average values for the entire pore space of the sample material instead of a single pore. $T_{2 S}^{-1}$ is still described by equation 4 , but $\rho_{2}$ and $S / V$ are average values. Current NMR theory associates the average $\rho_{2}$ with the paramagnetic species (i.e., unpaired electrons) on the surface of the pore space within the sampled material (Brownstein and Tarr, 1979; Godefroy et al., 2001).

To investigate the effect of the mineralogic form of $\mathrm{Fe}(\mathrm{III})$ on the NMR response of a geologic material, we conducted a laboratory study to compare the magnitude of the five averaged parameters $T_{2 M L}^{-1}, T_{2 B}^{-1}, T_{2 s}^{-1}, T_{2 D}^{-1}$, and $\rho_{2}$, for water-saturated samples containing pure quartz and five different iron oxides, with known concentrations of $\mathrm{Fe}(\mathrm{III})$. This allowed us to quantify changes in $T_{2}^{-1} M L$ with 
changes in mineralogy and determine which relaxation mechanism (bulk fluid, surface, or diffusion) was responsible for any observed change in $T_{2 M L}^{-1}$.

\section{METHODS AND MATERIALS}

\section{Materials and NMR sample preparation}

In order to determine the NMR response of the selected iron oxides, we prepared samples of quartz sand coated with the iron oxides. This allowed us to control the concentration of iron in the samples and prevented the iron oxides from forming aggregates. Quartz sand [99.995\%,>40 mesh, silicon (IV) dioxide, Alfa Aesar] was used in this study as an analog for a naturally occurring mineral surface. The quartz sand was rinsed with $10 \% \mathrm{HCl}$ deionized water $(18 \mathrm{M} \Omega \cdot \mathrm{cm})$ to remove paramagnetic species, then coated with the iron oxides: ferrihydrite, goethite, hematite, lepidocrocite, and magnetite.

The same general procedure was used to prepare quartz sand coated with ferrihydrite, goethite, hematite and lepidocrocite. First, the iron oxide was synthesized. Ferrihydrite was synthesized by the titration of $\mathrm{Fe}\left(\mathrm{NO}_{3}\right)_{3} \cdot 9 \mathrm{H}_{2} \mathrm{O}(0.4 \mathrm{~mol} / \mathrm{L})$ with $1 \mathrm{~mol} / \mathrm{L} \mathrm{NaOH}$ to $\mathrm{pH} 7$ (Hansel et al., 2003); goethite was synthesized by the slow oxidation of $\mathrm{FeCl}_{2} \cdot 4 \mathrm{H}_{2} \mathrm{O}$ solution (Schwertmann and Cornell, 2000); hematite was synthesized by the forced hydrolysis of $\mathrm{Fe}\left(\mathrm{NO}_{3}\right)_{3} \cdot 9 \mathrm{H}_{2} \mathrm{O}$ (0.4 mol/L) solution (Schwertmann and Cornell, 2000); and lepidocrocite was synthesized by the hydrolysis of $\mathrm{FeCl}_{2} \cdot 4 \mathrm{H}_{2} \mathrm{O}$ $(0.2 \mathrm{~mol} / \mathrm{L})$ kept at ph 6.7, using $1 \mathrm{~mol} / \mathrm{L} \mathrm{NaOH}$ (Schwertmann and Cornell, 2000). The synthesized iron oxides were then washed with deionized water to remove excess salts. Slurries composed of the synthesized iron oxides and deionized water were mixed with sand to obtain a mixture containing $1 \%$ iron by weight (calculated stoichiometrically); this mixture was allowed to dry. The iron-coated sands were subsequently washed three times with deionized water. Once coated, the mineral compositions of the synthesized iron oxides were confirmed using X-ray diffraction spectrometry.

Powdered, synthetic magnetite, in the form of synthetic iron oxide $\left(100 \% \mathrm{Fe}_{3} \mathrm{O}_{4}\right.$, Fisher Scientific), was chosen as an analog for naturally occurring magnetite. Then we followed the same procedure used with the other iron oxides to obtain quartz sands coated with magnetite. Initially, the magnetite and sand were mixed to contain $1 \%$ iron by weight; however, the relaxation time of the $1 \%$ mixture was found to be too short to measure. A mixture of magnetite and sand containing $0.65 \%$ iron by weight was prepared and used in this study.

\section{NMR measurement procedures}

Two samples of each type of sand (pure quartz and iron-coated) were packed into cylindrical Teflon sample holders of interior diameter $2.1 \mathrm{~cm}$ and height $6 \mathrm{~cm}$. The porosity of each sample, packed in the container, was measured with a Coberly-Steven Helium Pycnometer. Once the porosity had been measured, the sample was saturated under pressure with deionized water; this process took approximately 30 minutes. NMR measurements were made approximately 1 hour after the saturation of the samples.

NMR relaxation data were collected using a 2.2 MHz Maran Ultra NMR Core Analyzer (Resonance Instruments) using a CPMG pulse sequence. A single data point was obtained at each echo in the CPMG pulse sequence; 32,000 echoes were used. Data were collected at four echo times, $t_{E}=300,400,600$, and $800 \mu \mathrm{s}$, resulting in total times for each pulse sequence of 9.6, 12.8, 19.2, and 25.6 s. The data were stacked 100 times to improve the signal-to-noise ratio. The delay time between each pulse sequence was $10 \mathrm{~s}$ to ensure that the sample had returned to thermal equilibrium prior to the start of the pulse sequence. Measurements were consistently made at $30^{\circ} \mathrm{C}$.

Once the NMR measurements had been completed on the saturated samples, the pore water was removed from each sample by centrifuging and used to measure $T_{2 B}^{-1}$. It was only necessary to measure $T_{2 B}^{-1}$ at one value of $t_{E}$ because no internal magnetic field gradient is present in fluids; we chose $t_{E}=300 \mu \mathrm{s}$ as a representative echo spacing. Then, the NMR samples were dried overnight.

Three subsamples of each type of iron-coated sand were taken for surface area analysis; two of the subsamples from one NMR sample and one subsample from the other. Surface area measurements were also made on three samples of the quartz sand. The specific surface area $\left(S_{s}\right)$ of each sand, defined as the surface area normalized by the mass of the sample, was measured using a Beckman-Coulter SA3100 Analyzer, which produces accurate results for samples with a total surface area of $3 \mathrm{~m}^{2}$ or higher. For samples where $S_{s}$ was less then $0.2 \mathrm{~m}^{2} / \mathrm{g}$, the $S_{s}$ measurement was repeated using a Micromeritics ASAP 2020 Accelerated Surface Area and Porosimetry System, which produces accurate results for samples with a total surface area as low as $1 \mathrm{~m}^{2}$. All samples were measured using the Brunauer-Emmett-Teller (BET) adsorption method with $\mathrm{N}_{2}(\mathrm{~g})$ as the adsorbate.

\section{DATA ANALYSIS AND RESULTS}

\section{Porosity and surface area}

The measured porosity and specific surface area for each sample are given in Table 1 . The porosity ranged from 0.46 for the hematitecoated sand to 0.50 for the goethite-coated sand. These values are the same, within experimental error, as the porosity of the quartz sand sample (0.48) indicating that the addition of the iron coatings did not significantly change the porosity. For the sands coated with ferrihydrite, goethite, hematite, and lepidocrocite, the surface area increased significantly with the addition of the iron coating. The ferrihydrite-coated sand has a much higher surface area than the other iron-coated sands because of its amorphous crystal structure. The specific surface area of the quartz sand was not changed significantly by the addition of magnetite. $S / V$, also in Table 1 , was calculated from

Table 1. Physical property measurements porosity $(\phi)$, specific surface area $\left(S_{s}\right)$, and the calculated surfacearea-to-volume ratio $(S / V)$.

\begin{tabular}{lccc}
\hline Material & $\phi$ & $\begin{array}{c}S_{s} \\
\left(\mathrm{~m}^{2} / \mathrm{g}\right)\end{array}$ & $\begin{array}{c}S / V \\
(1 / \mu \mathrm{m})\end{array}$ \\
\hline Quartz sand & $0.48 \pm 0.02$ & $0.15 \pm 0.02$ & $0.48 \pm 0.06$ \\
Goethite-coated sand & $0.50 \pm 0.02$ & $0.58 \pm 0.09$ & $1.6 \pm 0.2$ \\
Lepidocrocite-coated & $0.49 \pm 0.02$ & $0.27 \pm 0.03$ & $0.75 \pm 0.08$ \\
sand & & & \\
Ferrihydrite-coated sand & $0.49 \pm 0.02$ & $2.5 \pm 0.5$ & $7.3 \pm 0.5$ \\
Hematite-coated sand & $0.46 \pm 0.02$ & $0.35 \pm 0.03$ & $1.09 \pm 0.09$ \\
Magnetite-coated sand & $0.48 \pm 0.02$ & $0.19 \pm 0.02$ & $0.48 \pm 0.02$
\end{tabular}




$$
\frac{S}{V}=m_{s} S_{s}\left(\frac{1}{V_{p}}\right),
$$

where $m_{s}$ is the total mass of the solid component, and $V_{p}$ is the volume of the pore space. $V_{p}$ was obtained from gravimetric measurements of the sample prior to and following saturation. Errors in the surface areas were calculated from repeated measurements and are attributed to the variability between samples of the same material.

\section{Sand sample relaxation rates, $T_{2 M L}^{-1}$}

Each NMR data set, from all the sand samples, displayed a multiexponential decay of magnetization (as described by equation 6). The data were fit to a distribution of 200 exponentially spaced $T_{2}$ values ranging from $1 \mathrm{~ms}$ to $10 \mathrm{~s}$ using the regularized nonnegative least-squares inversion routine developed by Whittall et al. (1991). This approach gives a less biased interpretation of the data than other commonly used fits (e.g., single-exponential, stretched-exponential, or double-exponential) because it does not specify the number of relaxation times, but instead allows any number of relaxation times between 0 and 200. The residual of each fit was examined to ensure that the noise was Gaussian and the data had not been overfit.

The $T_{2}$ distributions for the quartz sand and the iron-coated sands are shown in Figure 1 for $t_{e}=300 \mu \mathrm{s}$. The quartz sand $T_{2}$ distribution is a single, narrow peak; the $T_{2}$ distributions for the iron-coated sands are broader. The distributions for goethite-, lepidocrocite-, and ferrihydrite-coated sand are very similar in form with one dominant peak and other smaller peaks. The distribution of the hematite-coated sand is similar in form, but the peaks are not well resolved, and the amplitude of the dominant peak is lower than seen for the other three sands. The magnetite-coated sand has multiple unresolved peaks spread over several decades but does not contain a clear dominant peak.

The relaxation time distributions were used to determine $T_{2 M L}^{-1}$ for the samples. These values for $t_{E}=300 \mu$ s are given in Table 2. As expected, the presence of iron in a sample always results in a value for $T_{2 M L}^{-1}$ greater than $T_{2 M L}^{-1}$ for the pure quartz sand. However, as seen in Table 2, it is not simply the amount of iron present that can explain the magnitude of $T_{2 M L}^{-1}$. The ferrihydrite-, goethite-, hematite-, and lepidocrocite-coated sands all contained the same amount of iron, but have markedly different values for $T_{2 M L}^{-1}$. And the magnetite-coated sand, which contained the least amount of iron, has the greatest relaxation rate. In the following sections, we compare the averaged values $T_{2 B}^{-1}, T_{2 D}^{-1}$, and $T_{2 S}^{-1}$ to determine the mechanism (bulk fluid, surface, or diffusion) by which the change in the mineralogy of the samples has affected $T_{2 M L}^{-1}$.

\section{Bulk fluid relaxation rates, $T_{2 B}^{-1}$}

The NMR relaxation rates for the bulk fluids, extracted from the samples, are given in Table 2. To obtain these values of $T_{2 B}^{-1}$, the NMR relaxation data for the extracted bulk fluids were fit to a singleexponential decay using a least-squares algorithm. Analysis of the residuals indicated that a single-exponential decay was a valid assumption. The relaxation rate for deionized water was found to be $0.328 \mathrm{~s}^{-1}$. The values of $T_{2 B}^{-1}$ for the fluids from the quartz sand, the goethite-coated sand, and the hematite-coated sand were the same, within experimental error, as $T_{2 B}^{-1}$ of deionized water. The $T_{2 B}^{-1}$ values for the fluids from the ferrihydrite-coated sand, the lepidocrocitecoated sand, and the magnetite sand were $0.411 \mathrm{~s}^{-1}, 0.344 \mathrm{~s}^{-1}$, and $0.90 \mathrm{~s}^{-1}$, respectively. The increase in $T_{2 B}^{-1}$ from that of deionized water is most likely because of the presence of dissolved paramagnetic species or suspended mineral particles.
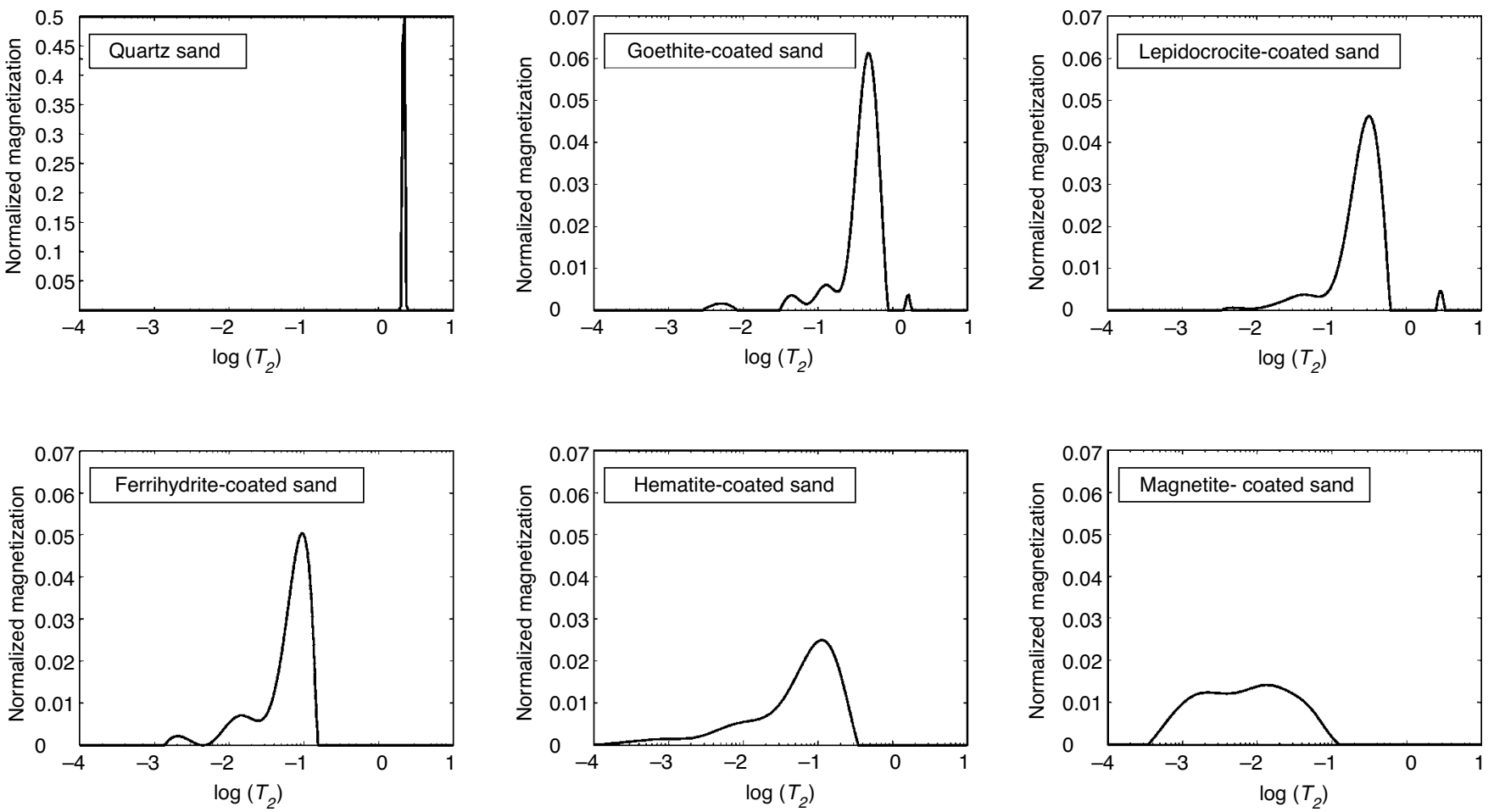

Figure 1. Normalized relaxation time distributions for quartz sand, ferrihydrite-coated sand, goethite-coated sand, hematite-coated sand, lepidocrocite-coated sand, and magnetite-coated sand. 


\section{Diffusion relaxation rates, $T_{2 D}^{-1}$}

The magnitude of the diffusion relaxation term, $T \frac{-1}{2 D}$, in the NMR response of a sample can be determined by measuring the dependence of $T_{2 M L}^{-1}$ on echo time $t_{E}$. As can be seen from equations 5 and 7, a plot of $T_{2 M L}^{-1}$ versus the square of the echo time $\left(t_{E}^{2}\right)$ will yield a straight line with slope equal to $D(\gamma G)^{2} / 12$. Figure 2 shows this plot for one sample of each of the iron-coated sands. The value of $T_{2 M L}^{-1}$ for the sands coated with ferrihydrite, goethite, hematite, and lepidocrocite shows negligible dependence on $t_{E}^{2}$, indicating that $D(\gamma G)^{2} / 12$ from equation 5 is zero, within experimental error. Given that $D$ and $\gamma$ are nonzero, this means that $G=0$ (i.e., there are no internal gradients in the magnetic field). The value of $T_{2 D}^{-1}$ for these samples, as given in Table 2, is zero.

$T_{2 M L}^{-1}$ for sand coated with magnetite shows a significant dependence on $t_{E}^{2}$. This indicates that the presence of magnetite causes internal gradients in the magnetic field $(G \neq 0)$, as expected given its high magnetic susceptibility. The value of $D(\gamma G)^{2} / 12$, obtained from the least squares fit of $T_{2 M L}^{-1}$ versus $t_{E}^{2}$, was used to calculate $T_{2 D}^{-1}$ according to equation 5 . At $t_{E}=300 \mu \mathrm{s}$, the value of $T_{2 D}^{-1}$ was $17 \mathrm{~s}^{-1}$ as shown in Table 2. It is clear from this that the presence of magnetite will result in a significant contribution to the measured relaxation rate from the diffusion relaxation mechanism.

\section{Surface relaxation rates, $T_{2 s}^{-1}$}

The final parameter to be assessed is the surface relaxation rate. The values of $T_{2 s}^{-1}$ determined using equation 7 for all samples are shown in Table 2. For the ferrihydrite-, goethite-, hematite-, and lepidocrocite-coated sands, and the quartz sand, $T_{2 s}^{-1}$ was calculated from equation 7 with $T_{2 D}^{-1}=0$. For the magnetite-coated sand, the relaxation rate extrapolated to $t_{E}^{2}=0$ (where $T_{2 M L}^{-1}=T_{2 B}^{-1}+T_{2 s}^{-1}$ ) was used to calculate $T_{2 s}^{-1}$. Values of $T_{2 s}^{-1}$ for the samples range from $0.16 \mathrm{~s}^{-1}$ for quartz sand to $125 \mathrm{~s}^{-1}$ for the magnetite-coated sand. As can be seen by reviewing these values in Table 2 , it is the variation in this term that is responsible for the variation seen in the NMR relaxation rates for the samples. In fact, the magnitude of this term is very close to the magnitude of $T_{2 M L}^{-1}$ for the ferrihydrite-, goethite-, hematite-, and lepidocrocite-coated sands.

The value of $T_{2 s}^{-1}$ for a material is determined by both $S / V$ and the surface relaxivity $\rho_{2}$. The values of $S / V$ are given in Table 1 , and the values of $\rho_{2}$, computed from $T{ }_{2 s}^{-1}$ using equation 4 , are given in Table 2. The values of $\rho_{2}$ range from $0.31 \mu \mathrm{m} / \mathrm{s}$ for quartz sand to $327 \mu \mathrm{m} / \mathrm{s}$ for magnetite-coated sand. The small $\rho_{2}$ for the quartz sand indicates that there are little to no paramagnetic species in this sand. Of specific interest is the observed variation in $\rho_{2}$ for the ironcoated sands. In this study, the variations in $\rho_{2}$ cannot be attributed to variation in the concentration of iron. The ferrihydrite-, goethite-, hematite-, and lepidocrocite-coated sands all contained an iron con-
Table 2. NMR relaxation rates and calculated relaxation parameters. Both $T_{2 M L}^{-1}$ and $T_{2 D}^{-1}$ for magnetite were calculated at $t_{E}=300 \mu \mathrm{s}$.

\begin{tabular}{lccccc} 
Material & $\begin{array}{c}T_{2 M L}^{-1} \\
\left(\mathrm{~s}^{-1}\right)\end{array}$ & $\begin{array}{c}T_{2 B}^{-1} \\
\left(\mathrm{~s}^{-1}\right)\end{array}$ & $\begin{array}{c}T_{2 S}^{-1} \\
\left(\mathrm{~s}^{-1}\right)\end{array}$ & $\begin{array}{c}T_{2 D}^{-1} \\
\left(\mathrm{~s}^{-1}\right)\end{array}$ & $\rho_{2}(\mu \mathrm{m} / \mathrm{s})$ \\
\hline $\begin{array}{l}\text { Quartz sand } \\
\begin{array}{l}\text { Goethite- } \\
\text { coated sand }\end{array}\end{array}$ & $0.49 \pm 0.02$ & $0.328 \pm 0.005$ & $0.16 \pm 0.02$ & 0 & $0.31 \pm 0.04$ \\
$\begin{array}{l}\text { Lepidocrocite- } \\
\text { coated sand }\end{array}$ & $4.4 \pm 0.1$ & $0.344 \pm 0.006$ & $4.1 \pm 0.2$ & 0 & $5.4 \pm 0.2$ \\
$\begin{array}{l}\text { Ferrihydrite- } \\
\text { coated sand }\end{array}$ & $16 \pm 1$ & $0.411 \pm 0.008$ & $16 \pm 2$ & 0 & $2.2 \pm 0.3$ \\
$\begin{array}{l}\text { Hematite- } \\
\text { coated sand }\end{array}$ & $20 \pm 2$ & $0.329 \pm 0.006$ & $19 \pm 2$ & 0 & $17.9 \pm 0.5$ \\
$\begin{array}{l}\text { Magnetite- } \\
\text { coated sand }\end{array}$ & $143 \pm 19$ & $0.90 \pm 0.04$ & $125 \pm 15$ & $17 \pm 2$ & $292 \pm 5$ \\
\hline
\end{tabular}

Figure 2. Plot of the transverse relaxation rate $\left(T_{2 M L}^{-1}\right)$ as a function of the square of the echo-time $\left(t_{E}^{2}\right)$ for ferrihydrite-coated sand, goethite-coated sand, lepidocrocite-coated sand, hematite-coated sand, and magnetite-coated sand.

centration of $1 \%$. The magnetite-coated sand contained $0.65 \%$ magnetite and, despite its lower iron content, has a very large $\rho_{2}$ value. The variation in $\rho_{2}$ most likely reflects a difference in parameters such as the density and distribution of paramagnetic species on the surface of the pores, the distance between the relaxing protons and the paramagnetic species, and the spin quantum number associated with the paramagnetic species. These parameters have been previously shown to have an effect on $\rho_{2}$ (Godefroy et al., 2001). Identifying and quantifying the parameters that are the fundamental cause of these variations is a topic of ongoing research, and requires characterizing the hydrated surface structure of each iron-oxide mineral.

\section{CONCLUSIONS}

The measurements from this study show conclusively that the NMR relaxation rate of a water-saturated sand is affected by both the presence and the mineralogical form of iron. The dominant effect of ferrihydrite, goethite, hematite, lepidocrocite, and magnetite was to increase $T_{2 S}^{-1}$, the magnitude of which is determined by $S / V$ and $\rho_{2}$. 
We found that the value of $\rho_{2}$ was very different for the different iron oxides; with additional research needed to determine the fundamental cause of these observed differences. The addition of magnetite to the quartz sand resulted in internal gradients and a contribution to $T_{2 M L}^{-1}$ from the diffusion relaxation mechanism

In order to use NMR relaxation rates to obtain accurate estimates of permeability in geologic materials, we need to be able to account for the effects of iron-oxide minerals. Although this laboratory study has been a start, we believe that it is necessary to explore a larger range of iron minerals and to further investigate the mechanisms controlling the changes in relaxation behavior that we observed. Quantifying the physical properties of minerals that lead to variations in relaxation rates will enhance our fundamental understanding of NMR relaxation and ultimately provide improved interpretation of NMR data.

\section{ACKNOWLEDGMENTS}

We would like to thank Jim Leckie and Gordon Brown for the use of their surface area analyzers, and Kate Tufano and Matt GinderVogel of the Stanford Environmental Soil and Biogeochemistry group for providing XRD measurements. We would also like to thank the associate editor for his/her handling of this paper, and the reviewers for their helpful comments. This work was supported in full by funding to Rosemary Knight under grant DE-FG0203ER15382-A0003 from the U.S. Department of Energy.

\section{REFERENCES}

Babes, L., B. Denizot, G. Tanguy, J. J. Le Jeune, and P. Jallet, 1998, Synthesis of iron oxide nanoparticles used as MRI contrast agents: A parametric study: Journal of Colloid and Interface Science, 212, 474-482.

Bloembergen, N., E. M. Purcell, and R. V. Pound, 1948, Relaxation effects in nuclear magnetic resonance absorption: Physical Review, 73, 679-715.

Brownstein, K. R., and C. E. Tarr, 1979, Importance of classical diffusion in NMR studies of water in biological cells: Physical Review A, 19, 24462453.

Bryar, T. R., C. J. Daughney, and R. J. Knight, 2000, Paramagnetic effects of iron(III) species on nuclear magnetic relaxation of fluid protons in porous media: Journal of Magnetic Resonance, 142, 74-85.

Carr, H. Y., and E. M. Purcell, 1954, Effects of diffusion on free precession in nuclear magnetic resonance experiments: Physical Review, 94, 630-638.

Cornell, R. M., and U. Schwertmann, 2003, The iron oxides: Properties, reactions, occurrences and uses: Wiley-VCH.
Foley, I., S. A. Farooqui, and R. L. Kleinberg, 1996, Effect of paramagnetic ions on NMR relaxation of fluids at solid surfaces: Journal of Magnetic Resonance, 123, 95-104.

Gillis, P., and S. H. Koenig, 1987, Transverse relaxation of solvent protons induced by magnetized spheres: Application to ferritin, erythrocytes and magnetite: Magnetic Resonance in Medicine, 5, 323-345.

Godefroy, S., J. P. Korb, M. Fleury, and R. G. Bryant, 2001, Surface nuclear magnetic relaxation and dynamics of water and oil in macroporous media: Physical Review E, 64, 21605-1-13.

Gossuin, Y., A. Roch, R. N. Muller, P. Gillis, and F. Lo Bue, 2002, Anomalous nuclear magnetic relaxation of aqueous solutions of ferritin: An unprecedented first-order mechanism: Magnetic Resonance in Medicine, 48 959-964.

Hansel, C. M., S. G. Benner, J. Neiss, A. Dohnalkova, R. K. Kukadapu, and S. Fendorf, 2003, Secondary mineralization pathways induced by dissimilatory iron reduction of ferrihydrite under advective flow: Geochimica et Cosmochimica Acta, 67, 2977-2992.

Hunt, C. P., B. M. Moskowitz, and S. K. Banerjee, 1995, Magnetic properties of rocks and minerals, in T. J. Ahrens, ed., Rock physics and phase relations: A handbook of physical constants: AGU Books Board, 189-204.

Korb, J. P., S. Godefroy, and M. Fleury, 2003, Surface nuclear magnetic relaxation and dynamics of water and oil in granular packings and rocks: Magnetic Resonance Imaging, 21, 193-199.

Legchenko, A., J. M. Baltassat, A. Beauce, and J. Bernard, 2002, Nuclear magnetic resonance as a geophysical tool for hydrogeologists: Journal of Applied Geophysics, 50, 21-46.

Meiboom, S., and D. Gill, 1958, Modified spin-echo method for measuring nuclear relaxation times: Review of Scientific Instruments, 29, 688-691.

Schwertmann, U., and R. M. Cornell, 2000, Iron oxides in the laboratory: Preparation and characterization: Wiley-VCH.

Seevers, D. O., 1966, A nuclear magnetic method for determining the permeability of sandstones: Transactions of the SPWLA 7th Annual Logging Symposium, Paper L.

Senturia, S. D., and J. D. Robinson, 1970, Nuclear spin-lattice relaxation of liquids confined in porous solids: Society of Petroleum Engineers Journal, 10, 237-244.

Shushakov, O. A., 1996, Groundwater NMR in conductive water: Geophysics, 61, 998-1006.

Timur, A., 1969, Pulsed nuclear magnetic resonance studies of porosity, movable fluid, and permeability of sandstone: Journal of Petroleum Technology, 21, 755-786.

Vogeley, J. R., and C. O. Moses, 1992, ${ }^{1} \mathrm{H}$ NMR relaxation and rock permeability: Geochimica et Cosmochimica Acta, 56, 2947-2953.

Whittall, K. P., M. J. Bronskill, and R. M. Henkelman, 1991, Investigation of analysis techniques for complicated NMR relaxation data: Journal of Magnetic Resonance, 95, 221-234.

Yaramanci, U., G. Lange, and M. Hertrich, 2002, Aquifer characterization using surface NMR jointly with other geophysical techniques at the Nauen/Berlin test site: Journal of Applied Geophysics, 50, 47-65.

Yilmaz, A., M. Bucciolini, G. Longo, F. Franciolini, L. Ciraolo, and R. Renzi, 1990, Determination of dependence of spin-lattice relaxation rate in serum upon concentration of added Fe by MRI: Clinical Physics and Physiology Measurements, 11, 343-439. 


\title{
A laboratory study of the effect of magnetite on NMR relaxation rates
}

\author{
Kristina Keating *, Rosemary Knight \\ Department of Geophysics, Stanford University, Mitchell Building, Stanford, CA, 94305, United States
}

Received 6 February 2007; accepted 8 September 2007

\begin{abstract}
We conducted a laboratory study to measure the effect of magnetite concentration and grain size on proton nuclear magnetic resonance (NMR) relaxation rates of sand mixtures and to determine the dominant mechanism by which relaxation occurs. We measured mixtures of quartz and three different forms of magnetite: a powdered synthetic magnetite; a small-grained, natural magnetite; and a large-grained, natural magnetite. The powdered synthetic magnetite was mixed with quartz in five concentrations ranging from 0.14 to $1.4 \%$ magnetite by weight; both sizes of natural magnetite were mixed with quartz in concentrations of 1 and $2 \%$ magnetite by weight. The NMR response of the water-saturated samples was measured and used to calculate four averaged relaxation rates for each magnetite concentration: the total mean log, bulk fluid, surface, and diffusion relaxation rates. The results of this study show that: 1) surface relaxation was the dominant relaxation mechanism for all samples except the powdered synthetic magnetite sample containing $1.4 \%$ magnetite; 2 ) the surface relaxivity is a function of the fraction of the surface area in the sample composed of magnetite; 3) there is no clear dependence of the diffusion relaxation rate on the concentration of magnetite.
\end{abstract}

(C) 2007 Elsevier B.V. All rights reserved.

Keywords: NMR; Magnetite; Transverse relaxation; Grain size

\section{Introduction}

Proton nuclear magnetic resonance (NMR) is a technique that can be used to detect the presence of hydrogen nuclei and to obtain information about their physiochemical environments. In a water-saturated geologic material the NMR relaxation measurement involves monitoring the relaxation, or return to equilibrium, of the bulk nuclear magnetization of the hydrogen nuclei in the pore water after the sample has been perturbed with a radiofrequency pulse. NMR relaxation measurements can be made in the laboratory or in the field using a well-logging device or a system deployed at Earth's surface. The well-logging system has been used for a number of years in the petroleum industry to determine reservoir permeability (e.g. Seevers, 1966; Timur, 1969; Korb et al., 2003) and has recently been used for hydrogeophysical applications (Clayton, 2006). The surfacebased system, referred to as MRS (magnetic resonance sounding),

\footnotetext{
* Corresponding author. Tel.: +1 650724 9939; fax: +1 6507257344 .

E-mail addresses: kkeat@stanford.edu (K. Keating),rknight@stanford.edu (R. Knight).
}

has been used to estimate hydraulic conductivity in the top $\sim 100$ m of Earth (e.g. Shushakov, 1996; Legchenko et al., 2002).

Of interest in our research is the effect of magnetite $\left(\mathrm{Fe}_{3} \mathrm{O}_{4}\right)$, a commonly occurring oxide, on the NMR relaxation measurement for geological materials. It is well known that the NMR relaxation rate of a material can be affected by the presence of paramagnetic ions such as $\mathrm{Fe}(\mathrm{III})$, and by contrasts in magnetic susceptibility between the pore water and the solid phase. Magnetite contains Fe (III) and has a large magnetic susceptibility, so it is not surprising that, in a study by Keating and Knight (2007), the addition of magnetite to quartz sand was found to have a significant effect on the relaxation rate. This previous study compared the relaxation behavior of five iron-oxide minerals including magnetite and found that the presence of magnetite had a much stronger effect on the relaxation rate than the presence of the other iron-oxides. The objective of this study was to extend the previous by determining the specific mechanism/s by which magnetite impacts the NMR relaxation rate.

We conducted laboratory experiments using quartz sand mixed with known concentrations of magnetite. We limited our measurements to magnetite concentrations of less than $2 \%$ 
which is representative of the magnetite concentrations found in natural environments (Thompson and Oldfield, 1986). Because magnetite can be present in soils as small crystals formed by biogeochemical processes (Oldfield, 1999; Evans and Heller, 2003) or as large grains from anthropogenic sources or physical weathering, we tested three grain sizes by using a powdered synthetic magnetite, a small-grained natural magnetite and a large-grained natural magnetite. These measurements advanced our understanding of the effect of magnetite on NMR relaxation data; knowledge that is essential if we are to obtain accurate information from NMR data in many geologic environments.

\section{Background and theory}

\subsection{NMR relaxation theory}

Proton NMR detects the presence of hydrogen nuclei; for hydrogeophysical applications the ability to detect hydrogen nuclei is of interest due to the presence of hydrogen in water. Each hydrogen nucleus, composed of a single proton, possesses a nuclear spin angular momentum. When in a static magnetic field, $B_{0}$, the nuclear spins in water precess about the static field. The nuclear spins precess at the Larmor frequency, $f_{0}$, which is related to $B_{0}$ by

$f_{0}=\gamma\left|B_{0}\right| /(2 \pi)$

where $\gamma$ is the gyromagnetic ratio for hydrogen protons in water molecules $(\gamma=0.267 \mathrm{rad} /[\mathrm{nT} \cdot \mathrm{s}])$. For MRS instruments $f_{0}$ ranges from 0.8 to $2.8 \mathrm{kHz}$; for NMR well-logging instruments $f_{0}$ ranges from 0.5 to $2 \mathrm{MHz}$; for most laboratory instruments $f_{0}$ ranges from 0.01 to $900 \mathrm{MHz}$. If a weak magnetic field oscillating at $f_{0}$ is applied the nuclear spins will tilt away from their equilibrium position. Once the oscillating field is removed the nuclear spins will return, or relax, to their equilibrium position. The return to equilibrium results in a measurable signal from the bulk nuclear magnetization, which can be described in terms of the transverse magnetization, $M_{x y}$.

For a bulk fluid the return to equilibrium behaves as an exponential decay:

$M_{\mathrm{xy}}(t)=M_{0} \exp \left(-t / T_{2 B}\right)$

where $M_{0}$ is the initial magnetization, $t$ is time and $T_{2 B}$ is the bulk fluid relaxation time; the inverse, $T_{2 B}^{-1}$, is referred to as the bulk fluid relaxation rate. $M_{0}$ is proportional to the total number of hydrogen nuclei in the bulk water. The magnitude of $T_{2 B}^{-1}$ for a fluid is controlled by dipole-dipole molecular interactions and depends on the viscosity of the fluid, the concentration of dissolved paramagnetic species (such as dissolved oxygen, $\mathrm{Mn}$ (II) ions or Fe(III) ions) and pH (Bloembergen et al., 1948).

For water confined in a pore, the measured relaxation rate is generally found to be greater than the bulk fluid relaxation rate due to two mechanisms that can enhance relaxation: surface relaxation and diffusion relaxation. In a single pore, the relaxation rate of water, $T_{2}^{-1}$, is described as a sum of relaxation rates (Brownstein and Tarr, 1979):

$T_{2}^{-1}=T_{2 B}^{-1}+T_{2 S}^{-1}+T_{2 D}^{-1}$ where $T_{2 S}^{-1}$ represents the surface relaxation rate and $T_{2 D}^{-1}$ represents the diffusion relaxation rate. Eq. (3) is valid for samples in the fast diffusion regime, which assumes that all protons travel to and interact with the solid surface within the time interval of the NMR experiment. For water in a porous geological material with a range of pore sizes, a multiexponential decay is observed,

$M_{\mathrm{xy}}(t)=\sum_{i} m_{i} \exp \left(-t / T_{2 i}\right)$.

Here $m_{i}$ is proportional to the number of moles of hydrogen relaxing with rate $T_{2 i}^{-1}$. The total number of moles of hydrogen is proportional to $M_{x y}(0)=\Sigma m_{i}$. The values of $m_{i}$ versus $T_{2 i}$ are often plotted to show the distribution of relaxation times. In studies of the NMR response of porous materials, the arithmetic mean of $\log T_{2 i}, T_{2 M L}$, is typically calculated from the distribution of relaxation times and used to represent the relaxation behavior. Eq. (3) then becomes:

$T_{2 M L}^{-1}=T_{2 B}^{-1}+T_{2 S}^{-1}+T_{2 D}^{-1}$

where rates are now taken to be average values for the entire pore space of the sample material instead of a single pore. While the magnitude of $T_{2 B}^{-1}$ is determined by the properties of the pore fluid, the magnitudes of $T_{2 S}^{-1}$ and $T_{2 D}^{-1}$ are strongly affected by the properties of the solid phase and controlled by very different relaxation mechanisms, referred to as the surface and diffusion relaxation mechanisms. Central to our research is the question of how the presence of magnetite influences these mechanisms. In the next sections we briefly review the way in which the properties of a geological material determine surface and diffusion relaxation rates.

\subsection{Surface relaxation}

The surface relaxation rate is determined by interactions that occur between the hydrogen nuclei in water and the solid surface of the geologic material. In the case of fast diffusion the surface relaxation rate is given by (Senturia and Robinson, 1970; Brownstein and Tarr, 1979),

$T_{2 S}^{-1}=\rho_{2} S / V$

where $S / V$ is the surface-area-to-volume ratio of the water-filled pore-space and $\rho_{2}$ is the surface relaxivity. For the fast diffusion assumption to be valid, the following relationship must be satisfied: $\rho_{2} V / S \ll D$, where $D$ is the self-diffusion coefficient of water $\left(D=2.5 \times 10^{-5}\right.$ for water at $\left.30^{\circ} \mathrm{C}\right)$. The relationship between the surface relaxation time and the surface-area-to-volume ratio shown in Eq. (6) is the basis for the use of NMR relaxation times to estimate permeability (e.g. Seevers, 1966; Timur, 1969) and hydraulic conductivity (e.g. Legchenko et al., 2002).

Current NMR theory associates $\rho_{2}$ with the presence of paramagnetic species (i.e. unpaired electrons) on the surfaces of the pore-space (Brownstein and Tarr, 1979; Godefroy et al., 2001). Laboratory studies of samples with known concentrations of paramagnetic ions, both in solid grains and adsorbed to 
the surface of solid grains, have shown that the surface relaxivity is proportional to the surface concentration of paramagnetic ions (Foley et al., 1996). Due to the presence of the paramagnetic species $\mathrm{Fe}(\mathrm{III})$ in magnetite we would expect $\rho_{2}$, and thus $T_{2 S}^{-1}$, of a sample to change as the concentration of magnetite in the sample changes.

\subsection{Diffusion Relaxation}

The diffusion relaxation rate is determined by the effect of the magnetic properties of the solid phase on the diffusing water molecules and is related to the average internal gradient of the magnetic field, $G$, by,

$T_{2 D}^{-1}=D\left(\gamma G t_{E}\right)^{2} / 12$

where $t_{E}$ is the echo-time, a rephasing parameter used during the Carr-Purcell-Meiboom-Gill (CPMG) pulse sequence (Carr and Purcell, 1954; Meiboom and Gill, 1958). (This pulse sequence was developed to rephase proton spins in a solid in the presence of non-uniform magnetic fields). Internal gradients are caused by a magnetic susceptibility contrast between the pore water and the solid phase. Because of the large magnetic susceptibility of magnetite even a small concentration of magnetite could result in the presence of internal gradients.

The presence of magnetic domains in magnetite causes the magnetic susceptibility to be grain size dependent. The magnetic domains are classified as superparamagnetic, single domain and multidomain. Although it is difficult to define the exact grain size separating the different domain states, in general, nano-size particles are superparamagnetic, single crystals with diameters ranging from 0.07 to $0.7 \mu \mathrm{m}$ are single domain, and massive grains with diameters greater than $10 \mu \mathrm{m}$ are multidomain (Hunt et al., 1995; Smith, 1999). Using these definitions the powdered synthetic magnetite samples used in this study were single domain grains; both grain sizes of the natural magnetite samples used in this study were multidomain grains.

\section{Methods and materials}

The objective of this study was to determine the relaxation mechanism/s responsible for the large effect of magnetite on the NMR relaxation rate of a geological material. That is, is the dramatically enhanced relaxation rate in the presence of magnetite due primarily to surface relaxation, diffusion relaxation, or both. To assess the contributions from the two relaxation mechanisms we conducted a laboratory experiment and compared the magnitude of the averaged relaxation rates, $T_{2 M L}^{-1}, T_{2 B}^{-1}, T_{2 S}^{-1}$, and $T_{2 D}^{-1}$ for water saturated samples containing known concentrations of magnetite. In addition to the NMR measurements, we also measured the surface area and magnetic susceptibility of the samples, two key parameters affecting $T_{2 S}^{-1}$ and $T_{2 D}^{-1}$.

\subsection{Preparation of magnetite and quartz mixtures}

The laboratory samples used in this study were prepared from mixtures of quartz sand and magnetite. Quartz sand
(99.995\% $\mathrm{SiO}_{2},>40$ mesh, silicon (IV) dioxide, Alfa Aesar) was used as an analog for a naturally occurring mineral surface. This quartz sand has previously been used for measurements in our laboratory and we have fully characterized its NMR response (Bryar and Knight, 2003; Keating and Knight, 2007). The quartz sand was rinsed with a weak acid solution $(10 \% \mathrm{HCl}$ and deionized water) to remove paramagnetic species then mixed with magnetite. A measurement on this quartz sand from the previous study by Keating and Knight (2007) was used in this study.

One type of magnetite used in this study was powdered synthetic magnetite, obtained from Fisher Scientific. The powdered synthetic magnetite was mixed with quartz sand to obtain samples containing $0.14,0.42,0.71,0.97$ and $1.4 \%$ magnetite by weight.

The other type of magnetite used in this study was in the form of grains derived from natural magnetite; these grains were prepared by Christina Trotter for use in a Master's thesis (Trotter, 2001). Two types of mixtures were prepared using the natural magnetite: small-grained natural magnetite and largegrained natural magnetite. The natural magnetite was initially in the form of large pieces of magnetite obtained from Pacific Mineral Museum (Vancouver, Canada). This magnetite was from a deposit in Texada, Gilles Bay, British Columbia, Canada. The magnetite was broken into cubic centimeter-sized pieces using a rock hammer then reduced in size using a stainless steel rock grinder. The magnetite was sieved for $30 \mathrm{~min}$ in copper sieves to isolate grain diameters of $110 \mu \mathrm{m}$ to $360 \mu \mathrm{m}$. A portion of these grains were used to create the largegrained natural magnetite mixtures. The remaining magnetite grains were further reduced in size using an alumina ceramic rock grinder. The magnetite was then sieved to isolate grain diameters of less than $45 \mu \mathrm{m}$; these grains were used to create the small-grained natural magnetite mixtures. Both sizes of natural magnetite grains were mixed with quartz sand to create samples containing 1 and $2 \%$ magnetite by weight. It was difficult to create samples with precisely 1 and $2 \%$ magnetite for the large-grained natural magnetite due to the large grain size and small concentration of this magnetite; the error on the magnetite concentration of these samples is higher than the error on the magnetite concentration of the samples containing small-grained natural magnetite and powdered synthetic magnetite.

\subsection{NMR measurement procedures}

Two NMR samples were prepared from each magnetite mixture by packing a weighed amount of the mixture into cylindrical Teflon sample holders of interior diameter $2.1 \mathrm{~cm}$ and height $6 \mathrm{~cm}$. The mass of each NMR sample ranged from 28.82 to $30.93 \mathrm{~g}$. Each sample was saturated with deionized water. The saturation process involved submerging the sample (in the sample holder) in a beaker of deionized water, placing the beaker in a vacuum chamber and reducing the pressure in the chamber to $75 \mathrm{mmHg}$ for $30 \mathrm{~min}$. This saturation process was repeated twice. NMR measurements were made one hour following saturation. 
NMR relaxation data were collected using a 2.2MHz Maran Ultra NMR Core Analyzer (Resonance Instruments) using a CPMG pulse sequence. The CPMG pulse sequence consists of applying a $90^{\circ}$ pulse followed by a series of $180^{\circ}$ pulses separated by $t_{E}$. A single data point was obtained at each echo in the CPMG pulse sequence; 32,000 echoes were used. Data were collected at six echo-times, $t_{E}=300,400,500,600,700$ and $800 \mu \mathrm{s}$, resulting in pulse sequence durations ranging from 9.6 to 25.6s. The data were stacked 100 times to improve the signal to noise ratio. A 10 s delay time between each pulse sequence was used to ensure that the sample had returned to thermal equilibrium prior to the start of the next pulse sequence. All NMR measurements were made at $30^{\circ} \mathrm{C}$.

Once the NMR measurements had been completed on the saturated samples the pore water was removed from each sample by centrifuging and the extracted water was used to measure $T_{2 B}^{-1}$. Data were collected at four echo times, $t_{E}=300$, 400,600 , and $800 \mu \mathrm{s}$. The NMR samples were then dried overnight.

\section{Sample characterization and material analysis}

\subsection{Porosity and surface area}

The measured porosity for each sample is given in Table 1. The porosity for each sample was calculated by

$\phi=V_{p} / V_{s}$

where $V_{p}$ is the volume of the pore space and $V_{s}$ is the known volume of the sample holder. $V_{p}$ was obtained from gravimetric measurements of the sample prior to and following saturation. The porosities ranged from 0.45 to 0.52 for both the synthetic and natural magnetite mixtures.

One subsample, approximately $33 \%$ by weight of the total sample, was taken from each NMR sample for surface area analysis. Surface area measurements were also made on two samples of both the synthetic and natural magnetite. The specific surface area, $S_{s}$, defined as the surface area normalized by the mass of the sample, was measured using a Micromeritics ASAP 2020 Accelerated Surface Area and Porosimetry System which produces accurate results for samples with a total surface area as low as $1 \mathrm{~m}^{2}$. All samples were measured using the Brunauer-Emmett-Teller (BET) adsorption method with $\mathrm{N}_{2}(\mathrm{~g})$ as the adsorbate.

The specific surface areas of the magnetite and quartz mixtures and the pure quartz sample are given in Table 1 . The specific surface area of the pure quartz sample is $0.15 \mathrm{~m}^{2} / \mathrm{g}$. The samples containing powdered synthetic magnetite have specific surface areas ranging from 0.18 to $0.25 \mathrm{~m}^{2} / \mathrm{g}$ where increases in $S_{\mathrm{s}}$ roughly correspond to increases in magnetite concentration. The specific surface areas of the samples containing smallgrained natural magnetite are $0.15 \mathrm{~m}^{2} / \mathrm{g}$ for the sample containing $1 \%$ magnetite and $0.18 \mathrm{~m}^{2} / \mathrm{g}$ for the sample containing $2 \%$ magnetite. The specific surface areas of the samples containing large-grained natural magnetite are $0.18 \mathrm{~m}^{2} / \mathrm{g}$ for the sample containing $1 \%$ magnetite and $0.19 \mathrm{~m}^{2} / \mathrm{g}$ for the sample containing $2 \%$ magnetite.

The $S_{S}$ measurements on the pure magnetite samples revealed that the $S_{S}$ values of the powdered synthetic magnetite were larger than the $S_{s}$ values of both sizes of the natural magnetite. The specific surface area of the synthetic magnetite was $6.6 \mathrm{~m}^{2} / \mathrm{g}$, the specific surface area of the small-grained natural magnetite was $1.1 \mathrm{~m}^{2} / \mathrm{g}$, and the specific surface area of the large-grained natural magnetite was $0.12 \mathrm{~m}^{2} / \mathrm{g}$.

The measured specific surface area was used to calculate $S / V$, also in Table 1, from

$S / V=m_{s} S_{S} / V_{p}$

where $m_{s}$ is the total mass of the solid component.

\subsection{Magnetic susceptibility measurements}

Three subsamples of each mixture were taken for magnetic susceptibility measurements: one subsample from each NMR sample and one sample from the remaining mixture. Each sample was packed into a box with a volume of $6 \mathrm{~cm}^{3}$. The mass specific magnetic susceptibility, $\chi_{m}$, was measured using a Sapphire SI-2 Susceptibility Instrument at the United States Geological Survey in Menlo Park, CA. This instrument produces a peak field of $1.0 \times$ $10^{-4} \mathrm{~T}$ at a frequency of $800 \mathrm{~Hz}$ and measures susceptibilities ranging from $10^{-6}$ to 1 in cgs units. Three measurements made on each sample were averaged to find $\chi_{m}$; prior to each measurement the baseline magnetic field was measured. An empty container was also measured to obtain a calibration factor which was used to correct for the geometry of the sample holder.

The measurements of mass specific magnetic susceptibility versus the magnetite concentration are shown in Fig. 1. The susceptibility meter returned $\chi_{m}$ in units of cgs per gram; the output $\chi_{m}$ was multiplied by $4 \pi \times 10^{-3}$ to obtain $\chi_{m}$ in $\mathrm{m}^{3} / \mathrm{kg}$. For all the magnetite mixtures, $\chi_{m}$ increases with increasing magnetite concentration and is in the range of $0.70 \times 10^{-6}$ to $15.0 \times 10^{-6} \mathrm{~m}^{3} / \mathrm{kg}$. For the powdered synthetic magnetite mixtures there is a distinct linear relationship between $\chi_{m}$ and magnetite concentration; this is predicted from mixture theories (Berryman, 1995; Crook et al., 2002). The error in the magnetic susceptibility measurements is below the size of the data point.

Table 1

Specific surface area $\left(S_{\mathrm{s}}\right)$, porosity $(\phi)$, and surface-area-to-volume ratio $(S / V)$ for the quartz and magnetite mixtures

\begin{tabular}{lllllllllll}
\hline & Quartz & \multicolumn{2}{l}{ Powdered, synthetic magnetite } & & \multicolumn{4}{c}{ Small-grained magnetite } & Large-grained magnetite \\
\hline Weight \% magnetite & 0 & 0.14 & 0.42 & 0.71 & 0.97 & 1.4 & 1 & 2 & 1 \\
$S_{S}\left(\mathrm{~m}^{2} / \mathrm{g}\right)$ & $0.15 \pm 0.02^{\text {a }}$ & $0.18 \pm 0.02$ & $0.18 \pm 0.02$ & $0.20 \pm 0.02$ & $0.24 \pm 0.02$ & $0.25 \pm 0.01$ & $0.15 \pm 0.01$ & $0.18 \pm 0.01$ & $0.18 \pm 0.02$ & $0.19 \pm 0.01$ \\
$\phi$ & $0.48 \pm 0.02^{\text {a }}$ & $0.45 \pm 0.02$ & $0.46 \pm 0.01$ & $0.52 \pm 0.02$ & $0.46 \pm 0.01$ & $0.46 \pm 0.03$ & $0.45 \pm 0.01$ & $0.46 \pm 0.01$ & $0.48 \pm 0.02$ & $0.48 \pm 0.01$ \\
$S / V\left(\mu \mathrm{m}^{-1}\right)$ & $0.48 \pm 0.06^{\mathrm{a}}$ & $0.58 \pm 0.04$ & $0.56 \pm 0.03$ & $0.54 \pm 0.02$ & $0.75 \pm 0.02$ & $0.79 \pm 0.02$ & $0.46 \pm 0.01$ & $0.56 \pm 0.02$ & $0.53 \pm 0.02$ & $0.57 \pm 0.02$ \\
\hline
\end{tabular}

${ }^{a}$ Data previously reported in Keating and Knight (2007). 


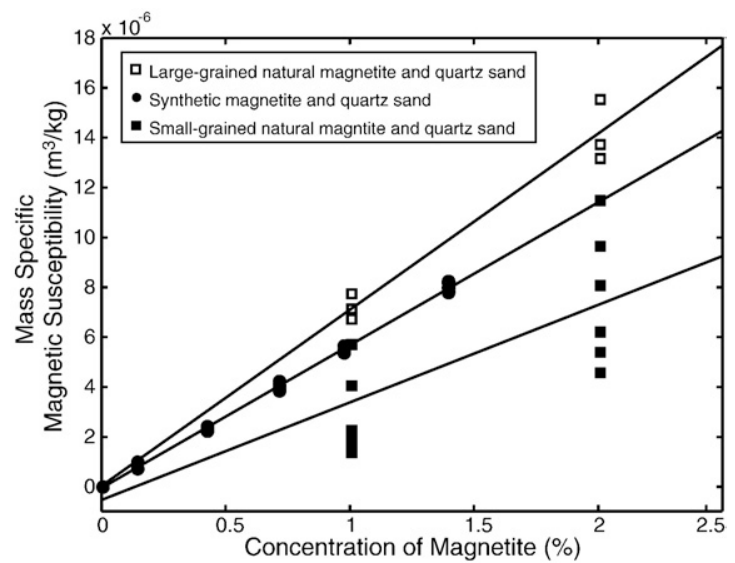

Fig. 1. Magnetic susceptibility versus the concentration of magnetite for the magnetite and quartz mixtures. The errorbars are within the size of the data point.

However, there is a large variability in $\chi_{m}$ for the small-grained natural samples consequently, $\chi_{m}$ for three additional samples of each concentration were measured. The large variability in these samples is attributed to the tendency of the small-grained natural magnetite particles to aggregate; this results in high levels of inaccuracy in the reported magnetite concentration. There is also variability in $\chi_{m}$ for the large-grained natural samples; this variability is due to the difficulty in preparing samples with precisely 1 and $2 \%$ magnetite for the large-grain natural magnetite due to the large grain size and small concentration of this magnetite. The differences between $\chi_{m}$ for the powdered synthetic magnetite and both sizes of the natural magnetite are likely due to differences in grain size, crystal shape, and impurities in the natural magnetite (e.g. titanium or aluminum substitution) (Dearing, 1999).

\section{NMR results}

Each NMR dataset from the water-saturated samples was fit to a distribution of 200 exponentially spaced $T_{2}$ values ranging from $1 \mathrm{~ms}$ to $10 \mathrm{~s}$ using the regularized non-negative leastsquares inversion routine developed by Whittall et al. (1991). This approach does not enforce a specific number of relaxation times but instead allows any number of relaxation times between 0 and 200. The relaxation time distributions measured at $t_{E}=300 \mu \mathrm{s}$ for all sample types as well as the relaxation time distribution for quartz sand measured in Keating and Knight (2007) are shown in Fig. 2. The relaxation time distributions for all the magnetite and quartz mixtures are broader than the relaxation time distribution for quartz sand. For both the powdered synthetic magnetite mixtures and the small-grained natural magnetite mixtures, the relaxation time distributions are broad, indicating that they consist of multiple super-imposed peaks. The relaxation time distributions for the large-grained natural magnetite mixtures are much narrower and have two super-imposed peaks separated by approximately one decade. The $T_{2 M L}^{-1}$ values for the samples were determined from the relaxation time distributions; we use these values to assess trends in the behavior of each sample.
The NMR datasets from the extracted fluids were fit using the regularized non-negative least-squares algorithm. The relaxation time distributions were found to either consist of a single relaxation time or to be narrow peaks. The $T_{2 B}^{-1}$ values, determined from the mean log average of the distributions, showed no dependence on echo time.

The $T_{2 M L}^{-1}$ and $T_{2 B}^{-1}$ values for the samples were used to calculate the $T_{2 S}^{-1}$ and $T_{2 D}^{-1}$ values. The magnitude of the $T_{2 S}^{-1}$ and $T_{2 D}^{-1}$ values were calculated by measuring the dependence of $T_{2 M L}^{-1}$ on echo time $t_{E}$ for each sample. As can be seen from Eqs. (5) and (7), a plot of $T_{2 M L}^{-1}$ versus the square of the echo-time, $t_{E}^{2}$, will yield a straight line with a slope equal to $D(\gamma G)^{2} / 12$ and an intercept equal to $T_{2 S}^{-1}+T_{2 B}^{-1}$. The $T_{2 M L}^{-1}$ values for all the mixtures show a significant dependence on $t_{E}^{2}$. This indicates that the presence of magnetite causes internal gradients in the magnetic field $(G \neq 0)$, as expected given its high magnetic susceptibility. The intercept obtained $\left(T_{2 S}^{-1}+T_{2 B}^{-1}\right)$ from the least squares fit of $T_{2 M L}^{-1}$ versus $t_{E}^{2}$ was used to calculate $T_{2 S}^{-1}$. The slope, $D(\gamma G)^{2} /$ 12 , of $T_{2 M L}^{-1}$ versus $t_{E}^{2}$, was used in Eq. (5) to calculate $T_{2 D}^{-1}$ at $t_{E}=300 \mu \mathrm{s}$.

The relaxation rates, $T_{2 M L}^{-1}, T_{2 B}^{-1}, T_{2 S}^{-1}$, and $T_{2 D}^{-1}$, are shown versus the magnetite concentration in Fig. 3 for the powdered synthetic magnetite mixtures and in Figs. 4 and 5 for the smalland large-grained natural magnetite mixtures respectively. Both the $T_{2 M L}^{-1}$ and the $T_{2 D}^{-1}$ values shown are at $t_{E}=300 \mu \mathrm{s}$. The error on each relaxation rate is the standard deviation calculated from repeated measurements; errors not shown are smaller than the data point. For all the magnetite mixtures, $T_{2 M L}^{-1}$ increases as the concentration of magnetite increases. The $T_{2 M L}^{-1}$ values also decrease with increasing grain size; the $T_{2 M L}^{-1}$ for the largegrained natural magnetite mixtures are an order of magnitude smaller than the $T_{2 M L}^{-1}$ values for the small-grained natural samples and two orders of magnitude smaller than the $T_{2 M L}^{-1}$ values for the powdered synthetic samples. In the following sections we will compare the $T_{2 B}^{-1}, T_{2 S}^{-1}$, and $T_{2 D}^{-1}$, values for the samples to determine the dominant relaxation mechanism/s and to further explore the differences between magnetite grain sizes.

Let us first consider the ways in which the relaxation rates, $T_{2 B}^{-1}, T_{2 S}^{-1}$ and $T_{2 D}^{-1}$, of the powdered synthetic magnetite mixtures, measured at $t_{E}=300 \mu$ s (shown in Fig. 3), vary with magnetite concentration. We make the following observations: 1) $T_{2 B}^{-1}$ does not show a dependence on magnetite concentration and accounts for less than $1 \%$ of the total relaxation rate; 2) $T_{2 S}^{-1}$ shows an overall increase with increasing magnetite concentration; 3) $T_{2 D}^{-1}$ shows little change with magnetite concentration for concentrations less than $1.4 \%$ and a large increase at a concentration of $1.4 \%$. When we consider the relative magnitude of $T_{2 S}^{-1}$ and $T_{2 D}^{-1}$, we find that for all mixtures the $T_{2 S}^{-1}$ values are larger than both the $T_{2 D}^{-1}$ and $T_{2 B}^{-1}$ values; $T_{2 S}^{-1}$ accounts for over $85 \%$ of the total relaxation rate for the mixtures containing less than $1.4 \%$ magnetite, and accounts for $67 \%$ of the total relaxation rate for the mixture containing $1.4 \%$ magnetite. As would be expected from Eq. (7), $T_{2 D}^{-1}$ increases as the echo time increases. At the largest echo time used in our measurements, $t_{E}=800 \mu \mathrm{s}$, the $T_{2 S}^{-1}$ values remain larger than both the $T_{2 D}^{-1}$ and $T_{2 B}^{-1}$ values and account for over $65 \%$ of the total relaxation rates for the mixtures containing less than $1.4 \%$ powdered synthetic 

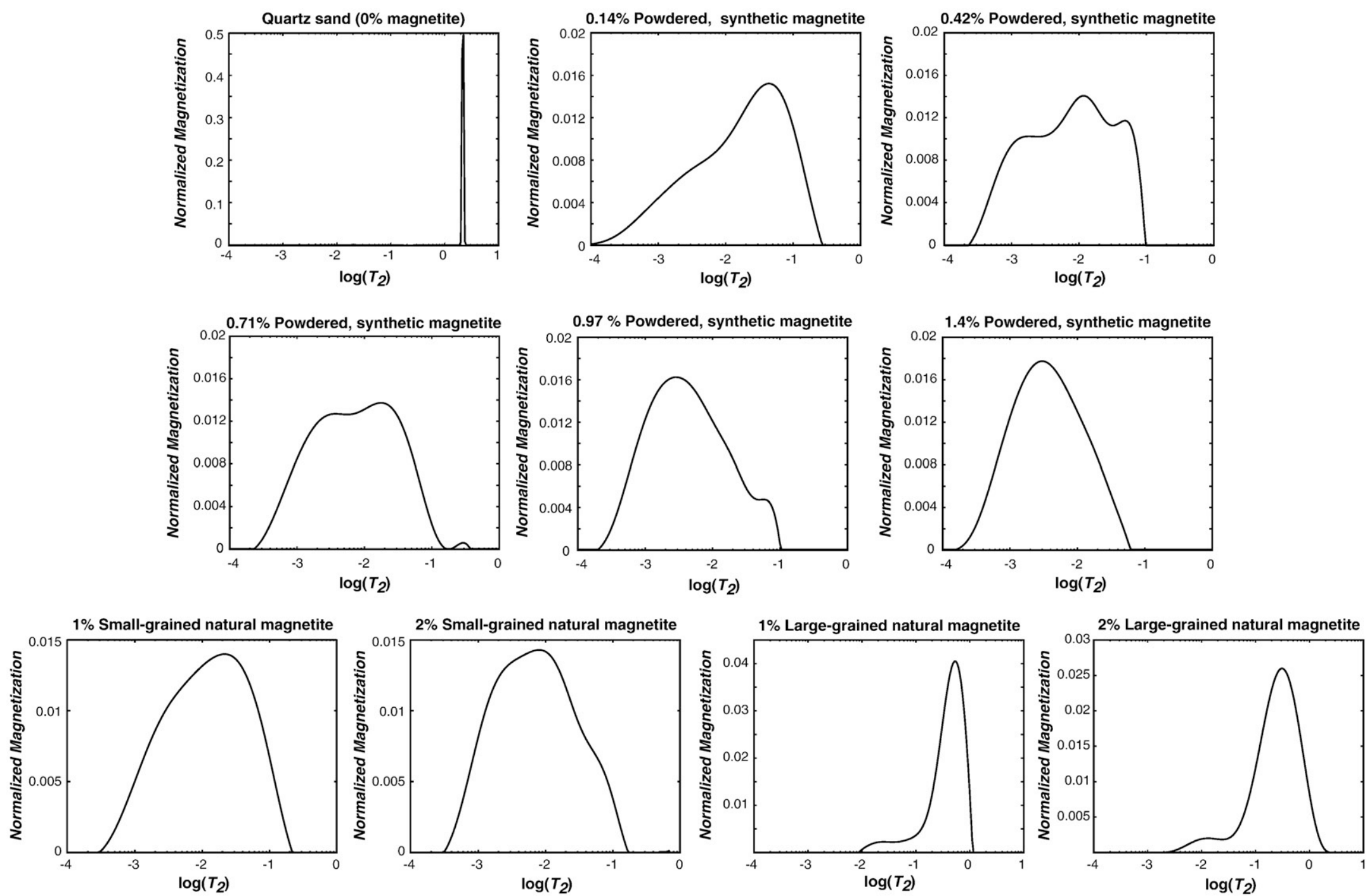

Fig. 2. Relaxation time distributions for the magnetite and quartz mixtures. The relaxation time distributions shown were measured at $t_{E}=300 \mu$ s. The quartz mixture shown was measured in Keating and Knight (2007). 


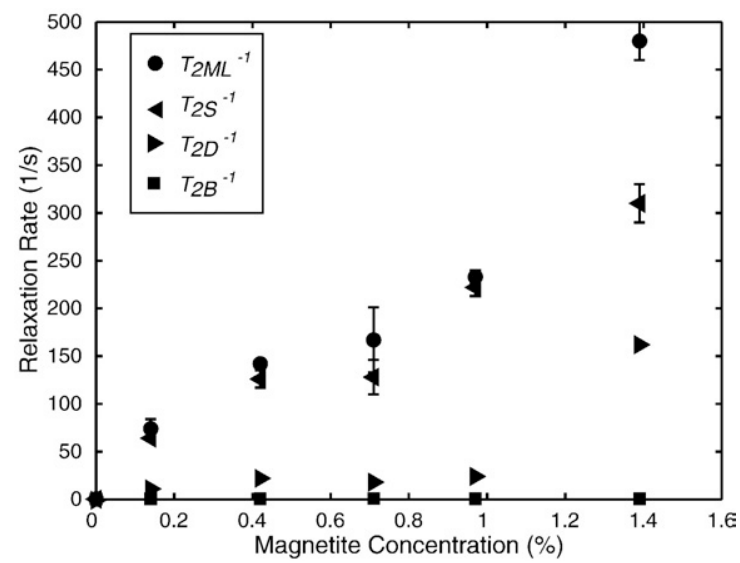

Fig. 3. Mean log average, surface, diffusion and bulk fluid relaxation rates versus magnetite concentration for water-saturated quartz mixed with powdered synthetic magnetite. Errorbars not shown are smaller than the size of the data point.

magnetite. For the mixture containing $1.4 \%$ powdered synthetic magnetite at $t_{E}=800 \mu \mathrm{s}, T_{2 S}^{-1}$ only accounts for $22 \%$ of the total relaxation time whereas $T_{2 D}^{-1}$ accounts for $78 \%$ of the total relaxation rate.

Let us next consider the ways in which the relaxation rates, $T_{2 B}^{-1}, T_{2 S}^{-1}$ and $T_{2 D}^{-1}$, of the small-grained natural magnetite mixtures, measured at $t_{E}=300 \mu \mathrm{s}$ (shown in Fig. 4), vary with magnetite concentration. We make the following observations: 1) $T_{2 B}^{-1}$ does not show a dependence on magnetite concentration and accounts for less than $1 \%$ of the total relaxation rate; 2) $T_{2 S}^{-1}$ increases with increasing magnetite concentration; 3) $T_{2 D}^{-1}$ decreases with increasing magnetite concentration. We find, as with the powdered synthetic magnetite mixtures, that the $T_{2 S}^{-1}$ values are consistently larger than the $T_{2 D}^{-1}$ and $T_{2 B}^{-1}$ values. At $t_{E}=300 \mu \mathrm{s} T_{2 S}^{-1}$ accounts for over $90 \%$ of each of the total relaxation rates. At an echo time of $800 \mu \mathrm{s}$, the contribution from the $T_{2 S}^{-1}$ values decreases but still accounts for over $50 \%$ of the

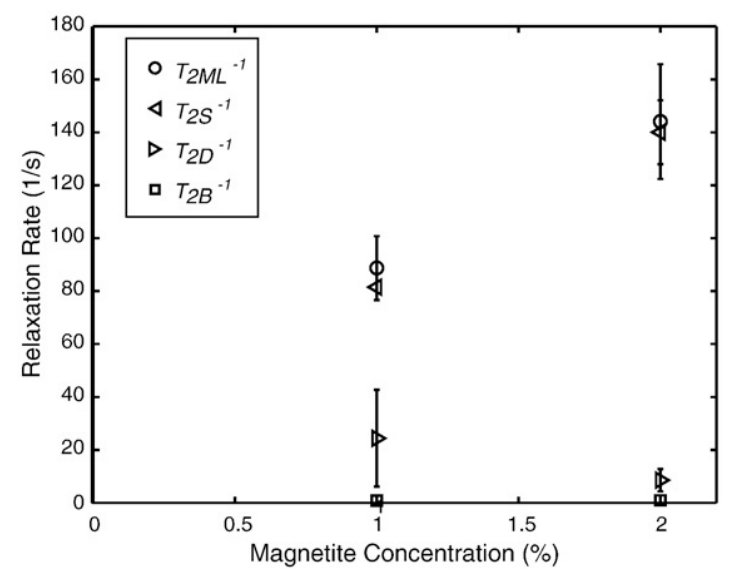

Fig. 4. Mean log average, surface, diffusion and bulk fluid relaxation rates versus magnetite concentration for water-saturated quartz sand mixed with small-grained natural magnetite. Errorbars not shown are smaller than the size of the data point. total relaxation rate and the $T_{2 D}^{-1}$ values increase to account for the remainder of the total relaxation time.

Finally, let us next consider the ways in which the relaxation rates, $T_{2 B}^{-1}, T_{2 S}^{-1}$ and $T_{2 D}^{-1}$, of the large-grained natural magnetite mixtures, measured at $t_{E}=300 \mu$ s (shown in Fig. 5), vary with magnetite concentration. 1) $T_{2 B}^{-1}$ shows no dependence on magnetite concentration within error of the measurement, and, in this case, $T_{2 B}^{-1}$ accounts for over $20 \%$, of the total relaxation rate; 2) $T_{2 S}^{-1}$ increases with increasing magnetite concentration; 3) $T_{2 D}^{-1}$ shows a small increases with increasing magnetite concentration. As seen for the other mixtures, $T_{2 S}^{-1}$ values are larger than the $T_{2 D}^{-1}$ and $T_{2 B}^{-1}$ values, accounting for over $70 \%$ of the total relaxation rates. At an echo time of $800 \mu \mathrm{s}$, the contribution from the $T_{2 S}^{-1}$ values does not decrease by much and still accounts for over $69 \%$ of the total relaxation rate. The $T_{2 D}^{-1}$ values only account for 0.5 to $2 \%$ of the total relaxation rate for $t_{E}=300$ to $800 \mu \mathrm{s}$.

To understand the effect of grain size on the relaxation rates we compare the values of $T_{2 B}^{-1}, T_{2 S}^{-1}$, and $T_{2 D}^{-1}$ for all the magnetite mixtures at a concentration of $1 \%$ for the natural magnetite mixtures and a concentration of $0.97 \%$ for the powdered synthetic magnetite mixtures we make the following observation: 1) $T_{2 B}^{-1}$ for all the mixtures is within 0.50 to $1.90 \mathrm{~s}^{-1}$ and does not show a dependence on grain size; 2) $T_{2 S}^{-1}$ shows a dependence on grain size increasing from $2 \mathrm{~s}^{-1}$ for the largegrained natural magnetite mixtures to $219 \mathrm{~s}^{-1}$ for the powdered synthetic magnetite mixtures; and 3) $T_{2 D}^{-1}$ does not show a strong correlation with grain size. The $T_{2 D}^{-1}$ values of the small-grained natural magnetite and the powdered synthetic magnetite mixtures are the same within error but are two orders of magnitude larger than the $T_{2 D}^{-1}$ values for the large-grained natural magnetite.

The large differences in $T_{2 S}^{-1}$ for the samples of different grain sizes does not correspond to changes in the surface-areato-volume ratios (Table 1) and so, as predicted in Eq. (6), must correspond to changes in $\rho_{2}$. The value of $\rho_{2}$ is proportional to the surface concentration of paramagnetic ions $\left(C_{p}\right)$ (Foley

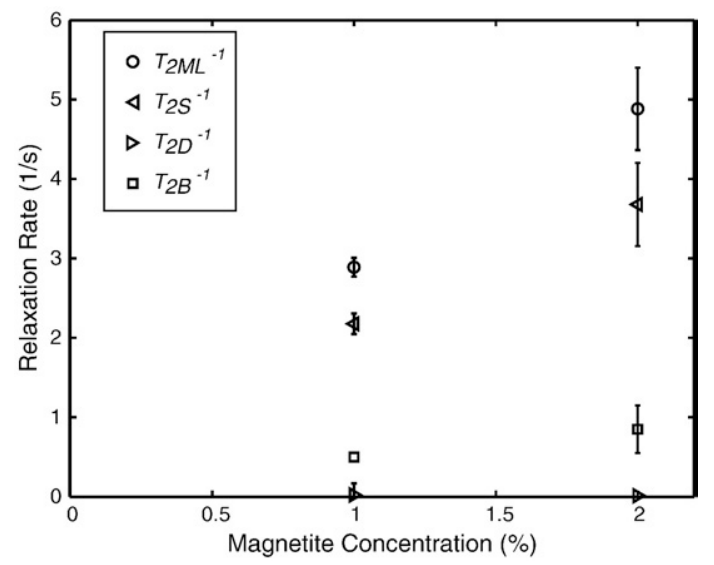

Fig. 5. Mean log average, surface, diffusion and bulk fluid relaxation rates versus magnetite concentration for water-saturated quartz mixed with largegrained natural magnetite. Errorbars not shown are smaller than the size of the data point. 


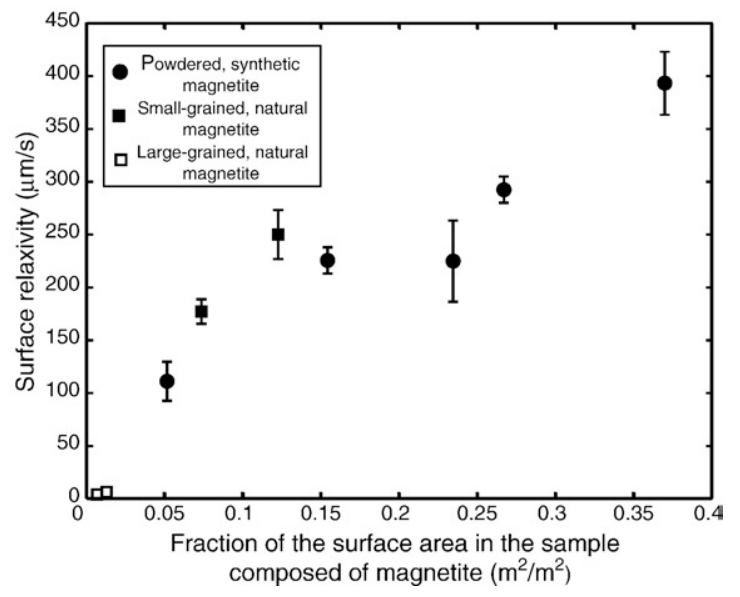

Fig. 6. Surface relaxivity versus the fraction of the surface area in the sample composed of magnetite for all the magnetite and quartz sand mixtures. Errorbars not shown are smaller than the size of the data point.

et al., 1996). This surface concentration can be represented as the fraction of the surface area containing paramagnetic ions. We can define $C_{p}$ as:

$C_{p}=\frac{S_{p}}{S_{T}}$

where $S_{p}$ is the surface area of the sample containing paramagnetic ions and $S_{T}$ is the total surface area of the sample. In our study $S_{p}$ can be assumed to be equivalent to the total surface area of magnetite present in the sample $\left(S_{M}\right)$ resulting in the following definition for $C_{p}$ :

$C_{p}=\frac{S_{M}}{S_{T}}$

The graph of $\rho_{2}$, calculated from Eq. (6), versus $C_{P}$ (Fig. 6) shows that $\rho_{2}$ increases with an increase in the fraction of the surface area in the sample composed of magnetite.

The $T_{2 D}^{-1}$ values show no trend with increasing magnetic susceptibility, as would be expected due to the relationship between $T_{2 D}^{-1}$ and $\chi_{m}$. We suspect that $T_{2 D}^{-1}$ is a function of the complex local magnetic field distribution within the pore space of a sample but leave it to another study to explore this relationship.

\section{Conclusion}

Using NMR relaxation rate measurements completed in this study we have determined the dominant relaxation mechanism for water-saturated sands in the presence of magnetite. We conclude that for all but one sample surface relaxation is the dominant relaxation mechanism. The exception is the powdered synthetic magnetite sample containing $1.4 \%$ magnetite; in this sample, at large echo times, the dominant relaxation mechanism is diffusion relaxation. Additionally, these results show that the surface relaxivity increases with an increase in total surface area of magnetite present in the sample per total surface area of the sample. Finally, these results did not find a strong correlation between diffusion relaxation rate and magnetite concentration or grain size.
While the data presented in this study have allowed us to recognize the link between the surface relaxivity and the fraction of the surface area in the sample composed of magnetite, further research is needed to understand, in more detail, the effect of magnetite on the surface and diffusion relaxation rates. One issue that needs to be addressed is the way in which the pore-scale distribution of the magnetite and its local effect on surface relaxivity affects the averaged surface relaxation rate and relaxivity calculated from the NMR relaxation time distributions. Another issue that remains to be addressed is the quantitative relationship between the local magnetic fields produced by the magnetite grains and the averaged diffusion relaxation rate calculated from the NMR relaxation time distributions.

Our research is focused on laboratory measurements designed to improve the understanding and interpretation of field measurements of NMR relaxation rates for environmental applications. We recognize that the precision of field measurements of NMR relaxation rates is currently limited due to technical issues associated with the available instruments. We are confident, however, that ongoing research to improve the field instrumentation will address these issues and lead to increased use of NMR as a valuable geophysical field method.

\section{Acknowledgments}

We would like to thank Christina Trotter for preparation of the natural magnetite samples, Gordon Brown for the use of his surface area analyzer, John Hillhouse for the use of the magnetic susceptibility analyzer, and the anonymous reviewers for their insightful comments. This work was supported in full by funding to Rosemary Knight under Grant DE-FG02-03ER15382-A0003 from the United States Department of Energy.

\section{References}

Berryman, J.G., 1995. Mixture theories for rock properties. In: Ahrens, T.J. (Ed.), Rock Physics and Phase Relations: A Handbook of Physical Constants. American Geophysical Union.

Bloembergen, N., Purcell, E.M., Pound, R.V., 1948. Relaxation effects in nuclear magnetic resonance absorption. Physical Review 73 (7), 679-715.

Brownstein, K.R., Tarr, C.E., 1979. Importance of classical diffusion in NMR studies of water in biological cells. Physical Review A 19 (6), 2446-2453.

Bryar, T.R., Knight, R.J., 2003. Laboratory studies of the effect of sorbed oil on proton nuclear magnetic resonance. Geophysical Research Letters 68 (3), 942-948.

Carr, H.Y., Purcell, E.M., 1954. Effects of diffusion on free precession in nuclear magnetic resonance experiments. Physical Review 94 (1), 630-638.

Clayton, N., 2006. Meeting oilfield developed geophysical logging in cased and uncased wells for high-resolution characterization of an alluvium ASR system in the Central Valley, California. Society of Exploration Geophysicists Hydrogeophysics Workshop. Vancouver.

Crook, N.P., Hoon, S.R., Taylor, K.G., Perry, C.T., 2002. Electron spin resonance as a high sensitivity technique for environmental magnetism: determination of contamination in carbonate sediments. Geophysical Journal International 149 (2), 328-337.

Dearing, J.A., 1999. Magnetic susceptibility. In: Walden, J., Oldfield, F., Smith, J. (Eds.), Environmental Magnetism: A Practical Guide. Technical Guide, 6. Quaternary Research Association, London, pp. 35-88. 
Evans, M.E., Heller, F., 2003. Environmental Magnetism: Principles and Applications of Enviromagnetics. Elsevier Science.

Foley, I., Farooqui, S.A., Kleinberg, R.L., 1996. Effect of paramagnetic ions on NMR relaxation of fluids at solid surfaces. Journal of Magnetic Resonance 123, 95-104.

Godefroy, S., Korb, J.-P., Fleury, M., Bryant, R.G., 2001. Surface nuclear magnetic relaxation and dynamics of water and oil in macroporous media. Physical Review E 64, 21605-1-21605-13.

Hunt, C., Moskowitz, B.M., Banerjee, S.K., 1995. Magnetic properties of rocks and minerals. In: Ahrens, T.J. (Ed.), Rock Physics and Phase Relations: A Handbook of Physical Constants. American Geophysical Union.

Keating, K., Knight, R., 2007. A laboratory study to determine the effect of ironoxides on proton NMR measurements. Geophysics 72 (1), E27-E32.

Korb, J.-P., Godefroy, S., Fleury, M., 2003. Surface nuclear magnetic relaxation and dynamics of water and oil in granular packings and rocks. Magnetic Resonance Imaging 21, 193-199.

Legchenko, A., Baltassat, J.-M., Beauce, A., Bernard, R., 2002. Nuclear magnetic resonance as a geophysical tool for hydrogeologists. Journal of Applied Geophysics 50, 21-46.

Meiboom, S., Gill, D., 1958. Modified spin-echo method for measuring nuclear relaxation times. Review of Scientific Instruments 29 (8), 688-691.

Oldfield, F., 1999. Environmental magnetism; the range of applications. In: Walden, J., Oldfield, F., Smith, J. (Eds.), Environmental Magnetism: A Practical Guide. Technical Guide, 6. Quaternary Research Association.
Seevers, D.O., 1966. A nuclear magnetic method for determining the permeability of sandstones. 7th SPWLA Transactions. Paper L.

Senturia, S.D., Robinson, J.D., 1970. Nuclear spin-lattice relaxation of liquids confined in porous solids. Society of Petroleum Engineers Journal 10, 237-244.

Shushakov, O.A., 1996. Groundwater NMR in conductive water. Geophysics 61 (4), 998-1006.

Smith, J., 1999. An introduction to the magnetic properties of natural materials. In: Walden, J., Oldfield, F., Smith, J. (Eds.), From: Environmental Magnetism: A Practical Guide. Technical Guide, 6. Quaternary Research Association.

Thompson, R., Oldfield, F., 1986. Environmental magnetism. Allen and Unwin.

Timur, A., 1969. Producible porosity and permeability of sandstones investigated through nuclear magnetic resonance principles. Log Analyst 10.

Trotter, C.E., 2001. Predicting the surface area to volume ratio of pores in iron rich sediments from nuclear magnetic resonance data. MSc Thesis. University of British Columbia.

Whittall, K.P., Bronskill, M.J., Henkelman, R.M., 1991. Investigation of analysis techniques for complicated NMR relaxation data. Journal of Magnetic Resonance 95, 221-234. 
Title:

Nuclear magnetic resonance relaxation measurements as a means of monitoring iron mineralization processes.

Authors:

$8{ }^{1}$ Department of Geophysics, Stanford University, Mitchell Building, Stanford, CA, 94305, USA

$9 \quad{ }^{2}$ Department of Geological and Environmental Sciences, Stanford University, Braun Hall, Building 320, Stanford, CA, 94305, USA

11 
17 In this laboratory study, we assessed the measurement of nuclear magnetic resonance

18 (NMR) relaxation times as a means of monitoring iron mineralization processes. We conducted

19 experiments in which columns containing ferrihydrite-coated quartz sand reacted with aqueous

$20 \mathrm{Fe}(\mathrm{II})$ solutions to form goethite, lepidocrocite and magnetite. An observed increase in the

21 volume of water relaxing with long relaxation times in the NMR relaxation time distribution

22 corresponds to the formation of goethite and lepidocrocite; a decrease in the average (mean log)

23 relaxation time, and a broadening of the relaxation time distribution, corresponds to the

24 formation of magnetite. These results indicate that NMR relaxation times are sensitive to

25 changes in iron mineralogy and illustrate the potential use of NMR for monitoring iron

26 mineralization processes.

28 Index Terms:

29 0925: Magnetic and electrical methods and properties, 1859: Rocks: physical properties, 30 1895: Instruments \& Techniques: Monitoring, 9810: New Fields

31 Keywords:

32 NMR, iron minerals, relaxation time 


\section{Introduction} chemistry, Earth sciences) to detect the presence of hydrogen nuclei $\left({ }^{1} \mathrm{H}\right)$ and determine their physiochemical environment. In the Earth sciences proton NMR $T_{2}$ spin-spin relaxation time measurements have been made for over 40 years in the petroleum industry, using instruments

41 lowered in wells to detect both hydrocarbons and water, and to determine the properties of oil

42 and gas reservoirs that affect the quantity and movement of fluids [e.g. Brown and Gamson,

43 1960; Kleinberg et al., 1992]. Over the past 15 years, a proton NMR device has been developed

44 that can be deployed from Earth's surface to obtain information about the properties of

45 groundwater aquifers [Legchenko et al., 2004]. Here we present the first study to demonstrate

46 that proton NMR can be used to monitor temporal changes in iron mineralization processes in

47 geologic materials. The ability to monitor such processes with non-invasive NMR measurements

48 would be very useful in laboratory studies of geochemical reactions. To be able to do the same in

49 subsurface environments using a surface-based NMR system would have a significant impact on

50 the way in which we design and manage the remediation of contaminated sites.

Iron (hydr)oxides are ubiquitous in the environment and, due to their high reactivity, can

53 strongly influence the mobility and bioavailability of organic and inorganic contaminants

54 [Cornell and Schwertmann, 2003]. Remediation techniques used to clean up contaminated sites,

55 such as biostimulation and bioaugmentation, often exploit the high reactivity of iron

56 (hydr)oxides to control and sequester contaminants [Lovely, 2001; Cornell and Schwertmann,

57 2003]. Monitoring the geochemical reactions associated with such remediation techniques is an 
58 effective way to monitor the progress of remediation. Current monitoring methods rely on direct

59 sampling, an approach that can be costly, incurs the risk of further spreading the contaminant,

60 and provides only localized information. Of interest in our research is identifying geophysical

61 techniques that could be used non-invasively, over large subsurface regions, to monitor the

62 geochemical reactions associated with contaminant remediation. We designed this laboratory

63 study to assess the use of proton NMR to monitor an important geochemical reaction likely to be

64 associated with any remediation strategy that involves changes in iron mineralogy.

65

66 2. Background

67

The measured NMR parameter was the proton NMR $T_{2}$ spin-spin relaxation time of water

69 in the pore space of a water-saturated sample of sand. During an NMR experiment, water

70 contained in a single pore will display an exponential decay in nuclear magnetization, $M$, as a

71 function of time, $t$,

72

$$
M(t)=M_{0} e^{-t / T_{2}}
$$

73 where $T_{2}$ is the spin-spin relaxation time and $M_{0}$ is the initial magnetization and is proportional to

74 the volume of water in the pore. In a water-saturated porous material, where water is contained in 75 many pores with different physiochemical environments, the relaxation behavior of the water is

76 described by a multiexponential decay,

$$
M(t)=\sum_{i} M_{0 i} e^{-t / T_{2 i}}
$$

78 where the initial magnetization for each relaxation time, $M_{0 i}$, is proportional to the volume of

79 water with the relaxation time $T_{2 i}$. The values of the initial magnetization, $M_{0 i}$, are plotted versus 
80 the relaxation times, $T_{2 i}$, yielding a distribution of relaxation times. The total initial

81 magnetization $M(0)$ is equal to $\sum_{i} M_{0 i}$ and is proportional to the volume of water in the

82 measured sample. The arithmetic mean of the log relaxation times in the observed distribution,

$83 T_{2 M L}$, is used to represent the average relaxation time.

In a previous study by Keating and Knight [2007] it was found that the NMR relaxation

times of water-saturated quartz sands varied significantly when the mineralogy of the iron

87 (hydr)oxide coating on the sand was changed. $T_{2 M L}$ was $0.0069 \pm 0.0009 \mathrm{~s}$ for magnetite-coated sand, $0.063 \pm 0.004 \mathrm{~s}$ for ferrihydrite-coated sand, $0.35 \pm 0.005 \mathrm{~s}$ for goethite-coated sand, and

$0.227 \pm 0.01 \mathrm{~s}$ for lepidocrocite-coated sand. In addition to the low relaxation time of the

90 magnetite-coated sand, this sample also displayed a $T_{2}$-distribution that was distinctly broader

91 than those obtained for ferrihydrite-, goethite-,and lepidocrocite-coated sand. Observations of

92 large differences in $T_{2 M L}$ values and differences in the relaxation time distributions led to the

93 following hypothesis: we can monitor a geochemical reaction that involves these iron minerals

94 by using NMR measurements.

$97\left(\mathrm{Fe}(\mathrm{OH})_{3} \cdot \mathrm{nH}_{2} \mathrm{O}\right)$, a poorly crystalline iron(III) (hydr)oxide that is an important precursor for

98 other iron (hydr)oxide minerals [Cornell and Schwertmann, 2003]. A common source of aqueous

$99 \mathrm{Fe}(\mathrm{II})$ in natural systems is microbial reduction of iron (hydr)oxides; the $\mathrm{Fe}(\mathrm{II})$ produced during

100 this process is involved in a series of reactions that can transform the remaining iron

101 (hydr)oxides. We used laboratory conditions under which aqueous Fe(II) is known to react with

102 ferrihydrite: a pH of 7.5 and an anaerobic environment [Hansel et al., 2003]. Under such 
103 conditions, the reaction of Fe(II) with ferrihydrite results in the formation of goethite,

104 lepidocrocite, and magnetite [Hansel et al., 2003]. Goethite and lepidocrocite form by the

105 dissolution of ferrihydrite and homogeneous reprecipitation of goethite and/or lepidocrocite

106 [Cornell and Schwertmann, 2003]. Magnetite predominantly forms by solid-state conversion of

107 ferrihydrite to magnetite; magnetite can also form by dissolution of goethite and lepidocrocite

108 and heterogeneous reprecipitation as magnetite. At Fe(II) concentrations less than $1.0 \mathrm{mmol}$

$109 \mathrm{Fe}(\mathrm{II}) / \mathrm{g}$ of ferrihydrite, goethite and lepidocrocite form; above this threshold $\mathrm{Fe}(\mathrm{II})$ concentration

110 magnetite forms [Hansel et al., 2003]. While factors such as $\mathrm{pH}$ and redox potential will

111 determine the rate and extent of this reaction and the mineralogy of the solid phases produced,

112 the supply rate and concentration of $\mathrm{Fe}$ (II) have been shown to be the most important controls on

113 the ferrihydrite transformation [Lovley et al. 1989; Hansel et al., 2003].

\section{3. Experimental Methods and Materials}

To create the starting material for the experiments, ferrihydrite was synthesized and

118 combined with quartz sand producing a ferrihydrite-coated sand with a solid phase concentration

119 of $1 \%$ iron (by weight). The ferrihydrite was synthesized by the method of Brooks et al. [1996]

120 which results in the formation of 2-line ferrihydrite; this method involves titration of a ferric

121 chloride solution with $\mathrm{NaOH}$ to a $\mathrm{pH}$ of 7.5. A series of experiments were then conducted

122 involving NMR measurements and/or chemical analysis on vertical columns $(0.7 \mathrm{~cm}$ diameter,

$12310 \mathrm{~cm}$ long) packed with the ferrihydrite-coated quartz sand, which was saturated, and reacted

124 with, an Fe(II) solution. Influent solutions were pumped from bottom to top through the columns

125 at a rate of $0.35 \mathrm{~mL} / \mathrm{min}$. For all experiments, deoxygenated water was pumped through the 
126 sample column for the first $2 \mathrm{~h}$ to ensure anaerobic conditions within the sand. After $2 \mathrm{~h}$ the

127 influent solution was changed to a Fe(II) solution containing ferrous chloride buffered to $\mathrm{pH} 7.5$

128 with PIPES (1,4-piperazinediethanesulfonic acid, $10 \mathrm{mM}$ ); solutions were prepared, stored in,

129 and pumped from a sealed glove bag held under anaerobic conditions by continuously purging

130 with $\mathrm{N}_{2}$. The effluent solution was collected at the top of the column for disposal.

To obtain NMR measurements, a vertical column of the ferrihydrite-coated quartz sand

133 was positioned in the NMR analyzer so that relaxation time measurements were spatial averages

134 over the entire column. Once the Fe(II) solution began entering the bottom of the column, NMR

135 relaxation time measurements were initiated and made approximately every 30 minutes.

NMR relaxation data were collected with a $2.2 \mathrm{MHz}$ Maran Ultra NMR Core Analyzer

138 (Resonance Instruments) using a Carr-Purcell-Meiboom-Gill (CPMG) pulse sequence [Carr and

139 Purcell, 1954; Meiboom and Gill, 1958]. The CPMG pulse sequence consists of applying a $90^{\circ}$

140 pulse followed by a series of $180^{\circ}$ pulses separated by the echo time, $t_{\mathrm{E}}$. A single data point was

141 obtained at each echo in the CPMG pulse sequence; 32000 echoes were used. Data were

142 collected at an echo time of $t_{E}=300 \mu \mathrm{s}$, resulting in a pulse sequence duration of $9.6 \mathrm{~s}$. The data

143 were stacked 100 times to improve the signal to noise ratio. A $10 \mathrm{~s}$ delay time between each

144 pulse sequence was used to ensure that the sample had returned to thermal equilibrium prior to

145 the start of the next pulse sequence. All NMR measurements were made at $30^{\circ} \mathrm{C}$.

147 Each measured decay curve was fit to a distribution of 200 exponentially-spaced $T_{2}$

148 values, ranging from $1 \mathrm{~ms}$ to $10 \mathrm{~s}$, using a regularized nonnegative least-squares inversion 
149 routine [Whittall and MacKay, 1989]. This approach gives a more objective interpretation of the

150 data than other commonly used fits (e.g. single-exponential, stretched-exponential or double-

151 exponential) because it does not specify the number of relaxation times but instead allows any

152 number of relaxation times between 0 and 200.

The first set of experiments was performed with an influent solution Fe(II) concentration

155 of $0.2 \mathrm{mM}$. Deoxygenated water, followed by the Fe(II) solution, was pumped through two

156 columns filled with the ferrihydrite-coated quartz sand positioned outside the NMR analyzer.

157 One column (referred to as Column 1a) was disassembled after 525 min (equivalent to an input

158 of $0.037 \mathrm{mmol} \mathrm{Fe(II))} \mathrm{and} \mathrm{the} \mathrm{other} \mathrm{column} \mathrm{(Column} \mathrm{1b)} \mathrm{was} \mathrm{disassembled} \mathrm{after} 1150 \mathrm{~min}$

159 (equivalent to an input of $0.080 \mathrm{mmol} \mathrm{Fe}(\mathrm{II})$ ). At the same time NMR relaxation times were

160 measured on a third column (Column 1c) filled with the ferrihydrite-coated quartz sand, as the

$161 \mathrm{Fe}(\mathrm{II})$ solution was pumped through the column, for a total of $1620 \mathrm{~min}$; this is equivalent to an

162 input of $0.110 \mathrm{mmol} \mathrm{Fe}(\mathrm{II})$. This column was then removed from the NMR analyzer and

163 disassembled. Reacted solids from all three columns were dried anaerobically and kept for

164 further analysis.

The second set of experiments was performed with an influent solution Fe(II)

167 concentration of $2.0 \mathrm{mM}$. NMR measurements were made on one column (referred to as Column

168 2a), as the $\mathrm{Fe}(\mathrm{II})$ solution was pumped through the column for one hour (equivalent to an input

169 of $0.042 \mathrm{mmol} \mathrm{Fe}(\mathrm{II}))$ at which time the column was disassembled. NMR relaxation times were

170 measured on a second column (Column 2b) filled with the ferrihydrite-coated quartz sand; Fe(II)

171 solution was pumped through the column for a total of 12 hours (equivalent to an input of 0.504 
$172 \mathrm{mmol} \mathrm{Fe(II))}$ at which time the column was disassembled; the last NMR measurement was made

173 at 10.4 hours (equivalent to an input of $0.436 \mathrm{mmol} \mathrm{Fe}(\mathrm{II})$ ). NMR measurements were repeated

174 on a third column (Column 2c) of sand for a total of 12 hours. The solid materials from columns

$1752 \mathrm{a}$ and $2 \mathrm{~b}$ were dried in an anaerobic environment and kept for further analysis.

For chemical analysis on the dried materials, we used the materials from bottom thirds of

178 the columns. Speciation and distribution of Fe phases were determined using X-ray absorption

179 spectroscopy (XAS) and X-ray diffraction (XRD). Samples for XAS and XRD analyses were

180 sonicated $(<4 \mathrm{~h})$ to remove the Fe from the sand, vacuum filtered onto a cellulose acetate filter to

181 form a homogenously distributed Fe layer, dried in the anaerobic chamber ( $\sim 30 \mathrm{~min})$, and then

182 sealed with Kapton polyimide film to prevent oxidation while minimizing X-ray absorption.

183 XAS and XRD analyses were conducted at beamlines 11-2 and 11-3 respectively at the Stanford

184 Synchrotron Radiation Laboratory (SSRL). A double crystal Si(220) monochromator was used

185 for energy selection. Scans were conducted from $100 \mathrm{eV}$ below to $1000 \mathrm{eV}$ above the Fe K-edge

186 at $7111 \mathrm{eV}$. EXAFS spectra were extracted from the averaged data files by pre-edge subtraction

187 followed by spline fitting using the SixPack XAS data analysis package [Webb, 2004].

188 Background-subtracted $k^{3}$-weighted EXAFS were analyzed using the SixPack interface to

189 IFEFFIT [Newville, 2001] and fitted in the $k$-range 3 to 14 . Linear combination of model

190 compounds was performed to reconstruct unknown spectra as previously described [Hansel et

191 al., 2003]; the detection limit for linear combination fitting was $\sim 5 \%$. The set of Fe reference

192 standards included ferrihydrite, goethite, lepidocrocite, magnetite, green rust-chloride, green

193 rust-sulfate, green rust-carbonate and hematite. Each of these materials was synthesized

194 following the procedures of Schwertmann and Cornell [1991]. 


\section{Discussion of Results}

Linear combination fits of EXAFS spectra indicate that, in both the $0.2 \mathrm{mM}$ and $2.0 \mathrm{mM}$

$198 \mathrm{Fe}(\mathrm{II})$ columns, ferrihydrite reacts with Fe(II) to form goethite, lepidocrocite and magnetite

199 (Figure 1, Table 1). These results were confirmed by X-ray diffraction. In both sets of

200 experiments, mineral transformations began prior to the start of NMR measurements. From the

201 proportion of each mineral formed in the various columns the following observations can be

202 made: (1) Goethite and lepidocrocite formed before magnetite. (2) In the $2.0 \mathrm{mM} \mathrm{Fe}(\mathrm{II})$ columns

203 the concentration of lepidocrocite and goethite formed was greater than that formed in the 0.2

$204 \mathrm{mM}$ Fe(II) columns. (3) In the $2.0 \mathrm{mM}$ Fe(II) columns magnetite was formed at the expense of

205 goethite and lepidocrocite.

Let us now consider the NMR data and determine whether these changes in iron

208 mineralogy are reflected in the $T_{2 M L}$ values and/or in the form of the relaxation time distributions.

209 We can use the NMR measurements on the same iron minerals reported in Keating and Knight

210 [2007] for comparison. While there will likely be some differences between the $T_{2 M L}$ values in

211 Keating and Knight [2007] and the values measured in this study (primarily due to differences in

212 the porosity and surface area of the samples) we are confident, after numerous experiments with

213 these minerals, that the relative magnitudes of the $T_{2 M L}$ values and the forms of the relaxation

214 time distributions will be the same. We therefore would expect to see an increase in $T_{2 M L}$ as

215 goethite and lepidocrocite are formed, then a dramatic decrease in $T_{2 M L}$ and a broadening of the

216 relaxation time distributions with the formation of magnetite 
The observed strong dependence of $T_{2 M L}$ for each column on the input of Fe(II) is the

219 main result of this study. This is shown in the plot in Figure 2, where the calculated $T_{2 M L}$ value

220 for each column has been normalized by the initial $T_{2 M L}$ value for the column. Also shown in this

221 figure are the points (in terms of input of $\mathrm{Fe}(\mathrm{II})$ ) at which the five columns were disassembled

222 and analyzed to determine the iron mineralogy. The $T_{2 M L}$ values for the $0.2 \mathrm{mM} \mathrm{Fe}(\mathrm{II})$ and the

$2232.0 \mathrm{mM} \mathrm{Fe}(\mathrm{II})$ columns all show the same general trend: there is a significant decrease in $T_{2 M L}$ as

224 the input of $\mathrm{Fe}(\mathrm{II})$ increases (i.e. as the reaction progresses) with $T_{2 M L}$ of Column $2 \mathrm{c}$ decreasing

225 by $\sim 80 \%$ with the highest input of $\mathrm{Fe}(\mathrm{II})(0.504 \mathrm{mmol})$. This large decrease in $T_{2 M L}$ is compatible

226 with an increase in the concentration of magnetite in the samples.

The changes in form of the full $T_{2}$-distributions, each normalized by the maximum value

229 of $M_{0 i}$ in the distribution, provide additional evidence of the link between the NMR data and the

230 geochemical reactions. The distributions for two columns are shown in Figure 3: the distributions

231 from Column 1c (the $0.2 \mathrm{mM} \mathrm{Fe}(\mathrm{II})$ column) are in Figure 3A and the distributions from Column

232 2c (a $2.0 \mathrm{mM} \mathrm{Fe}(\mathrm{II})$ column) are in Figure 3B. Shown in region (i) in each figure is the

233 normalized $T_{2}$-distribution for the ferrihydrite-coated sand saturated with deoxygenated water at

234 the start of the experiment; the distribution is repeated to enhance the display. The normalized

$235 T_{2}$-distributions for Columns $1 \mathrm{c}$ and $2 \mathrm{c}$ exhibit a trend towards broader peaks centered at shorter

236 relaxation times as the experiment progresses. The final normalized $T_{2}$-distribution for Column

237 2c, which corresponded to the maximum input of Fe(II) in the experiments, is very similar to the

238 normalized $T_{2}$-distribution for magnetite-coated sand from Keating and Knight [2007], shown in

239 region (ii) of Figure $3 \mathrm{~B}$. We conclude that the observed changes in the $T_{2}$-distributions, i.e. a

240 large overall decrease in the average relaxation time and the trend to broader distributions, 
241 provide further evidence that the NMR data are responding to the increase in the concentration of 242 magnetite within the columns.

The chemical analysis clearly indicates the presence of goethite and lepidocrocite (Table

246 Keating and Knight [2007], we would expect to see an increase in $T_{2 M L}$ as goethite and

247 lepidocrocite replace ferrihydrite. While we do not find an increase in $T_{2 M L}$ for any of the

248 columns, we do see a change in the data that we interpret to be an indication of the presence of

249 these two minerals. We see an increase in the volume of water relaxing with long relaxation

250 times in the measurements made on Columns $2 \mathrm{~b}$ and $2 \mathrm{c}$ at low $\mathrm{Fe}(\mathrm{II})$ input $(<0.1 \mathrm{mmol})$

251 chemical analysis of the materials from Column 2a shows the maximum observed concentration

252 of goethite and lepidocrocite at an Fe(II) input of $0.042 \mathrm{mmol}$.

To better examine this feature in the data, we binned the relaxation times, from each

255 distribution for Columns $2 \mathrm{~b}$ and $2 \mathrm{c}$, into long $\left(T_{2}>0.25 \mathrm{~s}\right)$, medium $\left(0.25 \mathrm{~s}>T_{2}>0.01 \mathrm{~s}\right)$, and

256 short $\left(T_{2}<0.01 \mathrm{~s}\right)$ relaxation times, thereby summing the corresponding $M_{0 i}$ values to obtain

257 three values, $M_{0 \text { long }}, M_{0 \text { medium }}, M_{0 \text { short }}$. For each measurement, the fractions of water relaxing at

258 "long", "medium" and "short" times are given by $M_{0 \text { long }} / M(0), M_{0 \text { medium }} / M(0), M_{0 \text { short }} / M(0)$,

259 respectively. We calculated the change in these three values as the experiment progressed. These

260 results are plotted in Figure 4 as a function of the input of Fe(II). Clearly seen in the plot is a

261 steady increase, as the experiment progresses, in $M_{0 \text { short }} M(0)$ and a corresponding decrease in

$262 M_{0 \text { medium }} / M(0)$. These changes are directly related to the key observations of our study - the

263 large overall decrease in $T_{2 M L}$, and the broadening of the relaxation time distribution (both of 
264 which are attributed to the formation of magnetite). The feature of interest appears at low Fe(II)

265 input $(<0.1 \mathrm{mmol})$, where there is a peak in $M_{0 \text { long }} / M(0)$. The relaxation times reported for

266 goethite- and lepidocrocite-coated sands by Keating and Knight [2007] are greater than those

267 reported for either ferrihydrite or magnetite. This peak is therefore consistent with the chemical

268 analysis which shows the formation of goethite and/or lepidocrocite followed by the formation of

269 magnetite, at the expense of goethite and/or lepidocrocite. A similar feature was not seen in the

270 relaxation time distributions for the $0.2 \mathrm{mM} \mathrm{Fe}(\mathrm{II})$ columns which have $\sim 40 \%$ lower

271 concentrations of goethite/lepidocrocite. Our interpretation is that the concentration of

272 goethite/lepidocrocite formed in those columns was not sufficient to cause a detectable increase

273 in $M_{0 \text { long }} / M(0)$.

274

275 5. Conclusions

276

NMR relaxation times were found to respond to changes in iron mineralogy during the

278 studied geochemical reaction. The dominant feature was the large overall decrease in $T_{2 M L}$ due to

279 the formation of magnetite. More subtle, but present in two columns, was a change in the

280 relaxation time distribution that can be attributed to the presence of goethite and lepidocrocite.

281 This study has demonstrated the potential use of NMR for monitoring iron mineralization

282 processes in the laboratory, and introduces the possibility of using a surface-based NMR system

283 to non-invasively monitor these same processes in the subsurface. 


\section{6. Acknowledgments}

289 This research was supported primarily by funding to Rosemary Knight under Grant DE-FG02-

290 03ER15382-A0003 from the United States Department of Energy. This research was also

291 supported under a National Science Foundation Graduate Research Fellowship provided to

292 Katharine Tufano. Portions of this research were carried out at the Stanford Synchrotron

293 Radiation Laboratory, a national user facility operated by Stanford University on behalf of the

294 U.S. Department of Energy, Office of Basic Energy Sciences. We thank Scott Fendorf and Sue 295 Brantley for their constructive comments on the manuscript.

296 


\section{References}

Brooks, S. C., D. L. Taylor, and P. M. Jardine (1996), Reactive transport of EDTA-complexed cobalt in the presence of ferrihydrite., Geochim. Cosmochim. Acta, 60, 1899-1908.

Brown, R. J. S. and B. W. Gamson (1960), Nuclear magnetism logging, Trans. Am. Inst. Min. Metall. Petr. Eng., 219 (8), 199-207.

Carr, H. Y., and E. M. Purcell (1954), Effects of diffusion on free precession in nuclear magnetic resonance experiments, Phys. Rev., 94, 630-638.

Cornell, R. M. and U. Schwertmann (2003), The Iron Oxides: Properties, Reactions, Occurrences and Uses, $2^{\text {nd }}$ ed, Wiley-VCH, location.

Hansel, C. M., S. G. Benner, J. Neiss, A. Dohnalkova, R. K. Kukadapu and S. Fendorf (2003), Secondary mineralization pathways induced by dissimilatory iron reduction of ferrihydrite under advective flow, Geochim. Cosmochim. Acta, 67, 2977.

Keating, K. and R. J. Knight (2007), A laboratory study to determine the effect of iron oxides on proton NMR measurements, Geophysics, 72, E27.

Kleinberg, R. L., A. Sezinger, D. D. Griffin, and M. Fukuhara (1992), Novel NMR apparatus for investigating an external sample, J. Mag. Res., 97 (3), 466.

316 Lovley, D. R., E. J. P. Phillips, and D. J. Lonergan (1989), Hydrogen and formate oxidation coupled to dissimilatory reduction of iron or manganese by alteromonas putrefaciens, Appl. Environ. Microbiol., 55, 700-706. 
319 Meiboom, S. and D. Gill (1958), Modified spin-echo method for measuring nuclear relaxation $320 \quad$ times, Rev. Sci. Instrum., 29, 688-691.

321 Newville, M. (2001), IFEFFIT: interactive XAFS analysis and FEFF fitting. Journal of

$322 \quad$ Synchrotron Radiation, 8, 322-324

323 Schwertmann, U., and R. M. Cornell (1991), Iron Oxides in the Laboratory; Weinheim: New $324 \quad$ York.

325 Webb, S. M. (2005), Sixpack: A graphical user interface for XAS analysis using IFEFFIT. $326 \quad$ Physica Scripta, T115, 1011-1014.

327 Whittall, K. P., and A. L. MacKay (1989), Quantitative interpretation of NMR relaxation data, $J$. 328 Mag. Res., 84, 134-152.

329

330 
339 Table 1: The concentrations (as the percent moles of Fe) of the iron minerals formed in the

340 bottom third of the 0.2 and $2.0 \mathrm{Fe}(\mathrm{II})$ columns. The percentages were determined using linear

341 combination fitting of Extended X-Ray Adsorption Fine Structure (EXAFS) spectra of Fe.

342

343

\begin{tabular}{lccc|cc} 
& \multicolumn{3}{c|}{$0.2 \mathrm{mM} \mathrm{Fe}(\mathrm{II})$ columns } & \multicolumn{2}{c}{$2.0 \mathrm{mM}$ Fe(II) columns } \\
& Column 1a & Column 1b & Column 1c & Column 2a & Column 2b \\
Material & $0.037 \mathrm{mmol}$ & $0.080 \mathrm{mmol}$ & $0.110 \mathrm{mmol}$ & $0.042 \mathrm{mmol}$ & $0.504 \mathrm{mmol}$ \\
\hline Ferrihydrite & $82 \%$ & $64 \%$ & $54 \%$ & $39 \%$ & $18 \%$ \\
Goethite & $18 \%$ & $15 \%$ & $16 \%$ & $42 \%$ & $21 \%$ \\
Lepidocrocite & $0 \%$ & $15 \%$ & $19 \%$ & $19 \%$ & $0 \%$ \\
Magnetite & $0 \%$ & $6 \%$ & $11 \%$ & $0 \%$ & $61 \%$ \\
\hline
\end{tabular}

344

345

346

347

348

349

350

351

352

353

354

355

356

357 
358 Figure 1: EXAFS spectra of the solid material from the bottom of the 0.2 and $2.0 \mathrm{mM}$ columns

359 shown along with the EXAFS spectra from the magnetite, lepidocrocite, goethite, and

360 ferrihydrite reference minerals. The data are represented with solid lines and the fits are indicated 361 with dotted lines.

362

363 Figure 2: Mean log relaxation time, normalized by the initial mean log relaxation time, versus 364 the input of Fe(II). Data are shown for the $0.2 \mathrm{mM} \mathrm{Fe}(\mathrm{II})$ column, Column 1c, and the three 2.0

$365 \mathrm{mM} \mathrm{Fe}(\mathrm{II})$ columns: Columns 2a, 2b, and 2c. The points at which columns were disassembled

366 (in terms of input of $\mathrm{Fe}(\mathrm{II})$ ) and the column numbers are indicated on the figure. Note that

367 Columns 1a and $1 \mathrm{~b}$ were only used for chemical analysis; no NMR measurements were made on 368 these columns.

370 Figure 3: The relaxation time distributions, normalized by the maximum value of $M_{0 i}$ in each 371 distribution. Data are shown for the $0.2 \mathrm{mM} \mathrm{Fe}(\mathrm{II})$ column, Column 1c (A), and the $2.0 \mathrm{mM}$

372 Fe(II) column, Column 2c (B). The section marked (i) in each figure contains the initial

373 distribution (repeated to enhance the display) obtained from measurements on the ferrihydrite-

374 coated sand saturated with deoxygenated water. Section (ii) of (B) shows the normalized $T_{2}$ -

375 distribution for water-saturated magnetite-coated sand as measured by Keating and Knight 376 [2007].

378 Figure 4: The change in $M_{0 \text { long }} / M(0), M_{0 \text { medium }} / M(0), M_{0 \text { short }} M(0)$ versus the input of Fe(II) 379 for Columns $2 \mathrm{~b}$ and $2 \mathrm{c}$. 
381 Figure 1:
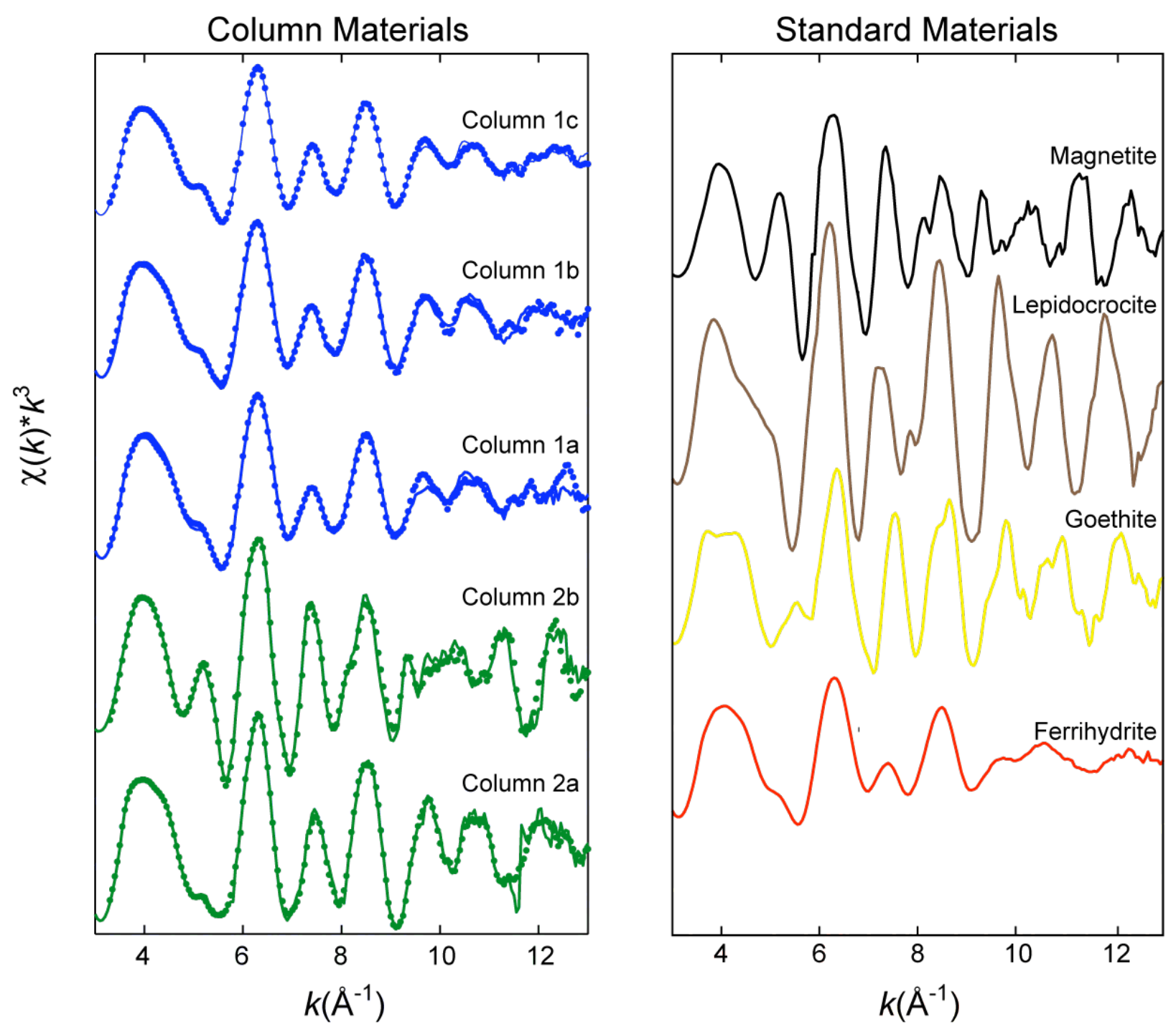

382

Figure 2:

384 


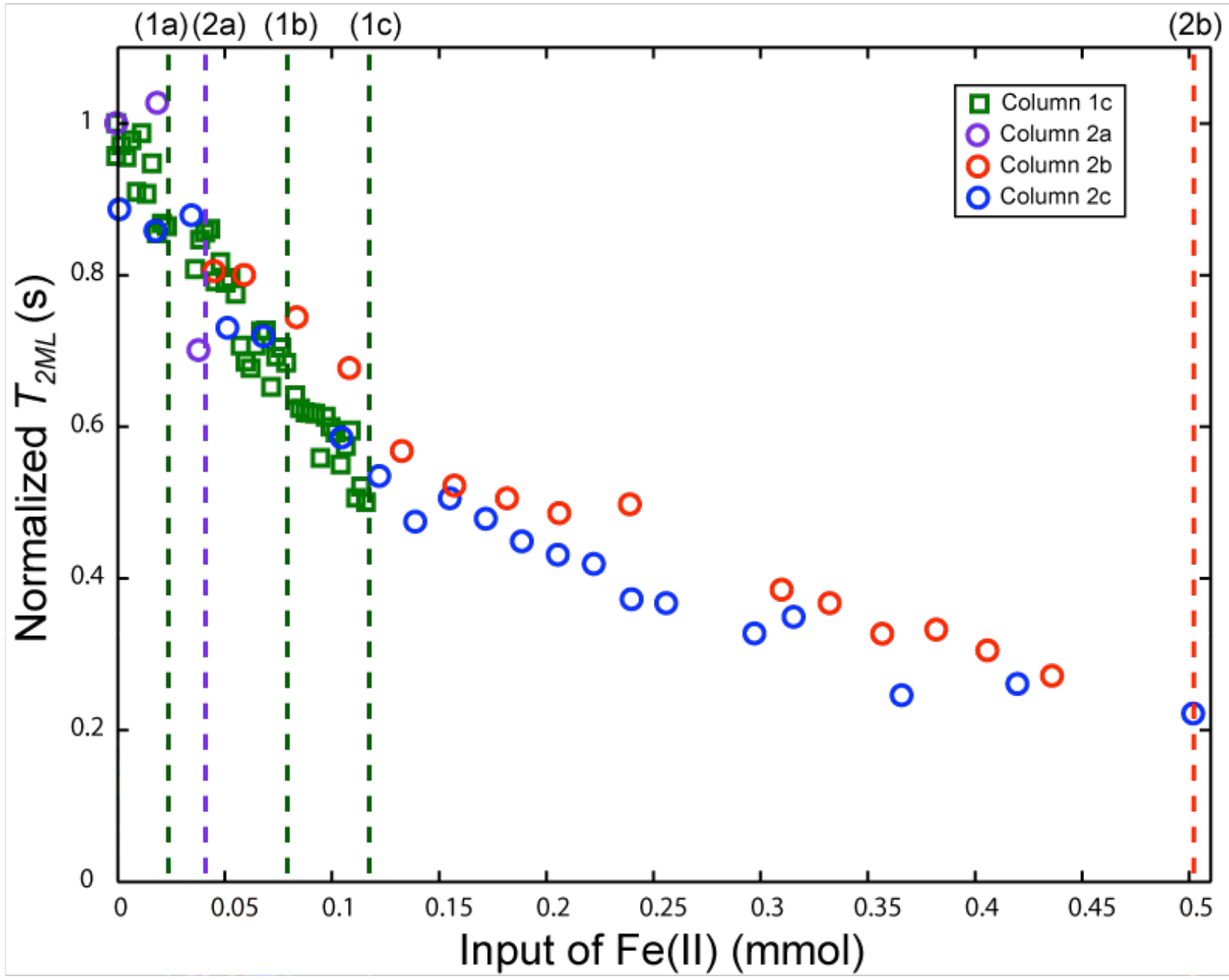

386 Figure 3:

(A)

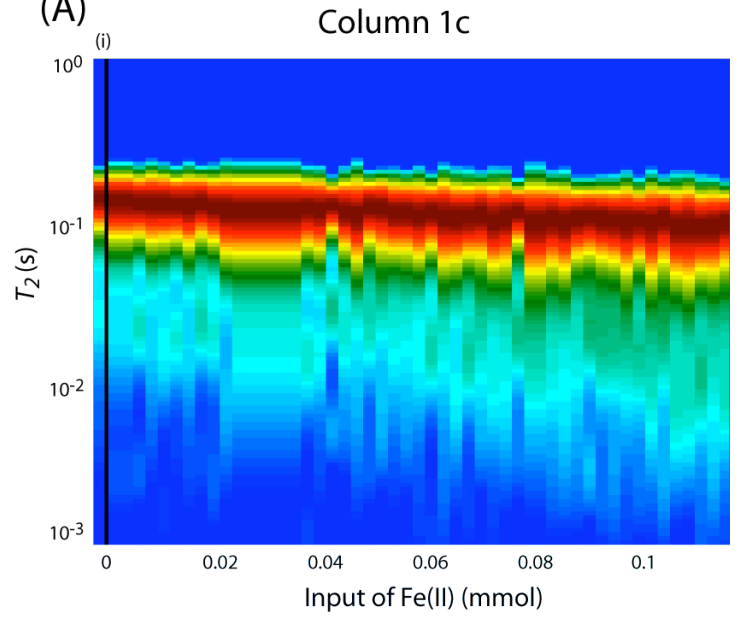

(B)

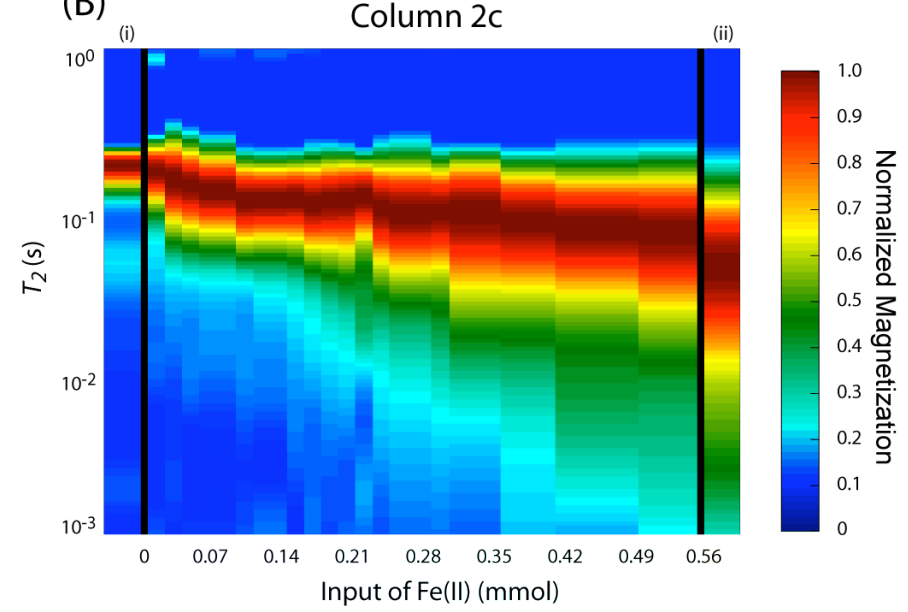

388 Figure 4: 


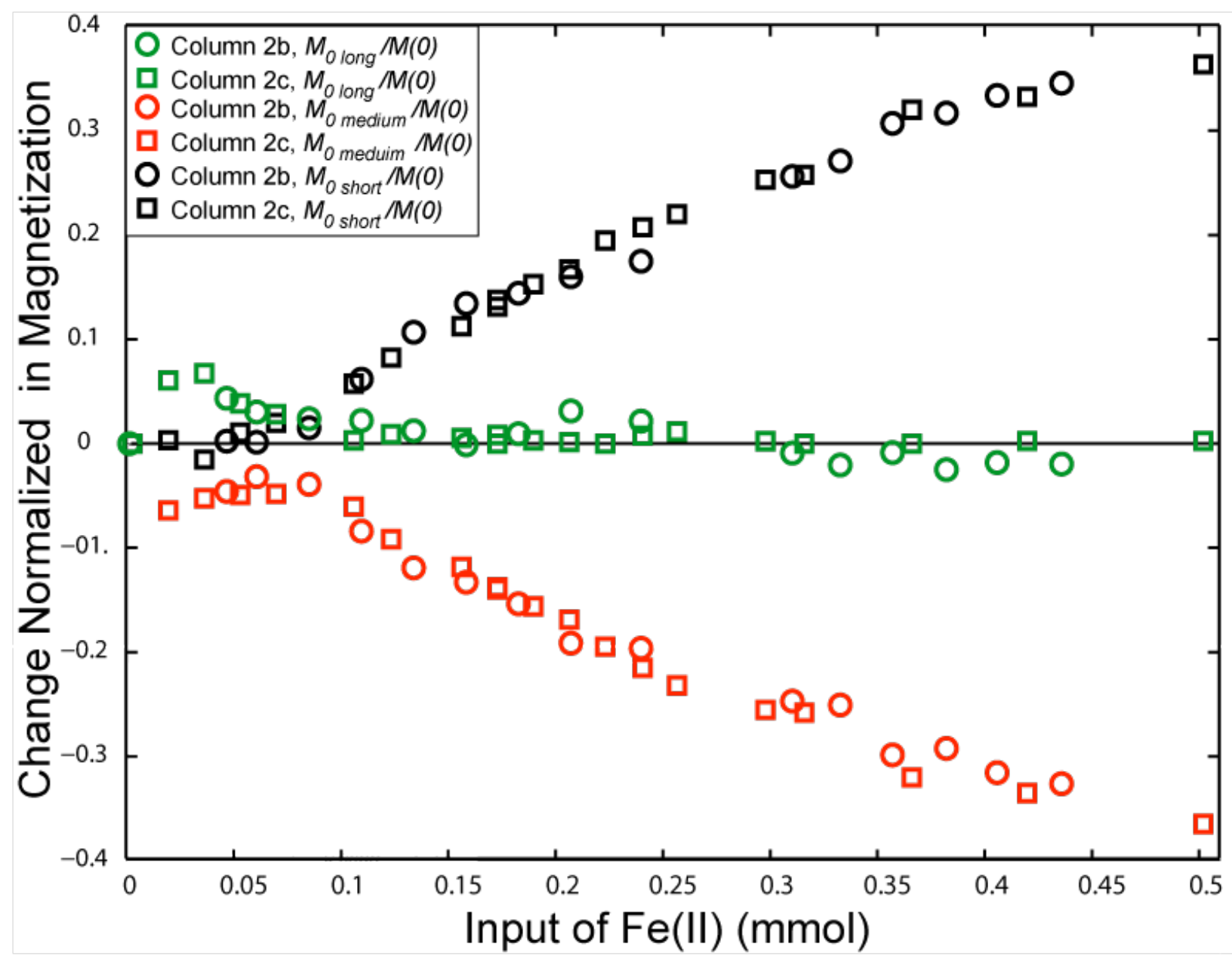

